\title{
Astrophysical Insights into Radial Velocity Jitter from an Analysis of 600 Planet-search Stars
}

\author{
Jacob K. Luhn ${ }^{1,2,5}$ (1D, Jason T. Wright ${ }^{1,2}$ (i) , Andrew W. Howard ${ }^{3}$ (i) , and Howard Isaacson ${ }^{4}$ (iD \\ ${ }^{1}$ Department of Astronomy, The Pennsylvania State University, 525 Davey Lab, University Park, PA 16802, USA; jluhn@ psu.edu \\ ${ }^{2}$ Center for Exoplanets and Habitable Worlds, 525 Davey Lab, The Pennsylvania State University, University Park, PA 16802, USA \\ ${ }^{3}$ Department of Astronomy, California Institute of Technology, Pasadena, CA, USA \\ ${ }^{4}$ Astronomy Department, University of California, Berkeley, CA, USA \\ Received 2019 October 23; revised 2020 March 2; accepted 2020 March 3; published 2020 April 27
}

\begin{abstract}
Radial velocity (RV) detection of planets is hampered by astrophysical processes on the surfaces of stars that induce a stochastic signal, or "jitter," which can drown out or even mimic planetary signals. Here, we empirically and carefully measure the RV jitter of more than 600 stars from the California Planet Search sample on a star by star basis. As part of this process, we explore the activity-RV correlation of stellar cycles and include appendices listing every ostensibly companion-induced signal we removed and every activity cycle we noted. We then use precise stellar properties from Brewer et al. to separate the sample into bins of stellar mass and examine trends with activity and with evolutionary state. We find that RV jitter tracks stellar evolution and that in general, stars evolve through different stages of RV jitter: the jitter in younger stars is driven by magnetic activity, while the jitter in older stars is convectively driven and dominated by granulation and oscillations. We identify the "jitter minimum"-where activity-driven and convectively driven jitter have similar amplitudes-for stars between 0.7 and $1.7 M_{\odot}$ and find that more-massive stars reach this jitter minimum later in their lifetime, in the subgiant or even giant phases. Finally, we comment on how these results can inform future RV efforts, from prioritization of followup targets from transit surveys like TESS to target selection of future RV surveys.
\end{abstract}

Unified Astronomy Thesaurus concepts: Radial velocity (1332); Exoplanet astronomy (486); Stellar astronomy (1583); Stellar phenomena (1619); Exoplanet detection methods (489)

Supporting material: machine-readable tables

\section{Introduction}

Since the first discoveries of planets orbiting other stars over a quarter century ago (Campbell et al. 1988; Latham et al. 1989; Wolszczan \& Frail 1992; Mayor \& Queloz 1995), more than 4000 exoplanets have been confirmed, with a multitude of planet candidates waiting to be confirmed. ${ }^{6}$ In the early days of planet detections, the radial velocity (RV) method was the primary method of discovery. With the advent of the Kepler mission (Borucki et al. 2010), the field exploded, and the transit method has become the dominant discovery method.

In spite of this, the importance of RV measurements has not diminished. Rather, RVs have become increasingly important because of their role in transit follow-up. In addition to the confirmation of planet detections via rejection of the overwhelmingly large amount of false positives, by combining the radius from transit detections with the mass from confirmed RV detections, we can begin to make inferences about the bulk composition of exoplanets (Weiss \& Marcy 2014; Dressing et al. 2015). However, RV resources are already struggling to keep up with transit discoveries. Despite the recent retirement of the Kepler spacecraft, additional planets are still being discovered with data from both the original Kepler mission as well as the extended K2 mission (Howell et al. 2014). Further, with TESS currently performing its $2 \mathrm{yr}$ primary mission (Ricker et al. 2014) and having recently been approved for an extended mission through 2023, the number of transiting

\footnotetext{
5 NSF Graduate Research Fellow.

6 http://exoplanetarchive.ipac.caltech.edu
}

planets can only be expected to continue to grow faster than $\mathrm{RV}$ teams can reasonably follow them up.

It is therefore critical that we understand the astrophysical drivers of stellar RV variability, or "jitter," so as to better understand which types of stars present poor cases for RV followup due to increased stochastic stellar signals. In the era of nextgeneration extremely precise spectrographs (HPF, EXPRESS, ESPRESSO, NEID) that can achieve sub- $\mathrm{m} \mathrm{s}^{-1}$ instrumental precision, the largest hurdle to finding Earth-like planets that remains is the intrinsic stellar jitter. With a stronger astrophysical understanding of RV jitter, we can avoid wasting time and resources on targets whose RV variations are not likely to permit efficient Doppler work. Intrinsic stellar jitter represents a fundamental limit for finding the smallest planets- those that are of most interest to the exoplanet community — and by knowing what physical characteristics are driving stellar RV jitter, we can find ways to overcome it, be it observationally, computationally, or statistically.

RV surveys have largely avoided stars that show signs of high levels of chromospheric activity, e.g., the Eta-Earth Survey (Howard et al. 2009). For Sun-like dwarf stars, measurements of chromospheric emission in the calcium $\mathrm{H} \& \mathrm{~K}$ lines, such as $\log R_{\mathrm{HK}}^{\prime}$ and $S_{\mathrm{HK}}$ (Noyes et al. 1984; Duncan et al. 1991), have been shown to correlate with intrinsic stellar RV variations (Campbell et al. 1988; Saar et al. 1998; Santos et al. 2000; Wright 2005). In these cases, the magnetic activity in the star that manifests as chromospheric emission serves as a proxy for the presence of spots or faculae on the stellar photosphere, which suppress or enhance the convective blueshift and also introduce rotationally modulated inhomogeneities. As a star ages on the main sequence, it loses angular momentum via magnetic winds 
and spins down. The result is a decrease in magnetic dynamo, a decrease in magnetically powered features on the surface of the star, and therefore a lowered chromospheric emission (Wilson 1963; Kraft 1967; Skumanich 1972). For stars whose RV jitter is dominated by magnetic activity, it is clear that older, "quieter" stars are the most amenable to RV observations.

However, work by Bastien et al. (2014b) has shown that even among "quiet" stars, RV jitter can be quite large. In fact, the RV jitter in their sample showed a clear increase with decreasing log $g$. That is, as a star evolves off the main sequence into the subgiant regime, its jitter increases substantially. This dependence on evolutionary state among subgiants was seen as early as Wright (2005) and Dumusque et al. (2011) and was even predicted both in Kjeldsen \& Bedding (1995), who used an analytic formula to describe the RV jitter due to p-mode oscillations of evolved stars, and again in Kjeldsen \& Bedding (2011), who provided a scaling for $\mathrm{RV}$ jitter due to granulation. ${ }^{7}$ Indeed, the relation seen in Bastien et al. (2014b) used a photometric measurement that probes granulation power, suggesting that for these stars, the primary driver of $\mathrm{RV}$ variations was granulation. The increase in granulation-induced RV variation with evolution is explained by the increase in size of a granular region as the surface gravity, and therefore surface pressure, decreases as the star expands (Schwarzschild 1975). As a result, the total number of granular regions across the face of the star decreases dramatically and so the degree to which the RVs from regions of rising and sinking gas balance out over the disk-integrated face of the star decreases because each individual granular region is more strongly weighted (Trampedach et al. 2013). Similarly, p-mode oscillation amplitudes increase as a star evolves and so oscillation-induced $\mathrm{RV}$ variation should also increase with evolution.

As we show below, over the course of a stellar lifetime, we therefore have periods where the RV jitter is dominated by different phenomena: the activity-dominated and the convectiondominated regimes. Convection is at least partially responsible for RV jitter even in this so-called "activity-dominated" regime as convection is a requirement for generating a magnetic dynamo, which is ultimately responsible for the RV variations (Parker 1955; Haywood et al. 2016). However, as both the granulation and oscillation components of RV jitter increase with evolutionary state, we treat these as different phases of the "convection-dominated" regime and compare it to the "activitydominated regime," where RV jitter decreases with evolution. It is reasonable then to expect that there is a "sweet spot" in a star's evolution where the combination of these two contributions is minimized. There is as of yet no published study that marries these two regimes and empirically investigates the evolutionary dependence of RV jitter. The goal of this work is to meticulously determine the astrophysical jitter due to stellar phenomena of more than 600 California Planet Search (CPS; Howard et al. 2010) stars for which we have reliably derived stellar parameters.

Previous empirical investigations of RV jitter using the CPS sample (e.g., Wright 2005; Isaacson \& Fischer 2010) have taken different approaches toward measuring RV jitter. For instance, Wright (2005) accounted for long-term linear trends present in the RVs by measuring the jitter about a linear fit for every star, but first removed stars from the sample that had known companions or showed evidence of

\footnotetext{
Further evidence of an evolutionary dependence on intrinsic RV variations was seen among giant stars by Hatzes \& Cochran (1998) and with a much larger sample in Hekker et al. (2008).
}

companions. Isaacson \& Fischer (2010) removed neither planets nor long-term linear trends and instead noted that they were most interested in the floor of RV jitter, as many stars would have jitter that included unsubtracted components. In this work, our sample is small enough to treat each star on a case-by-case basis to ensure that the RV jitter adequately reflects the intrinsic stellar RV jitter, yet still large enough to observe bulk trends across a wide range of stellar parameters. We are therefore able to analyze each star individually to account for cases of companions, long-term linear trends, and other effects that can typically inflate the reported RV jitter. Additionally, we have the benefit of many more years of observations, which are crucial for subtracting companions and long-term linear trends, allowing for more accurate measurements of RV jitter.

In Section 2, we describe the sample selection and RV observations made by the Keck-High Resolution Echelle Spectrometer (HIRES). We also describe the stellar properties used in this analysis, specifically focusing on the surface gravities and the activity metrics we use in our analysis. In Section 3, we describe our calculation of RV jitter, including detailed steps on our careful vetting process and theoretical scaling of the convective components of RV jitter. Section 4 highlights the main results of our empirical jitter calculations, investigating relations between activity, evolution, and mass. Section 5 contains a discussion of our results and places them in the context of RV surveys. We summarize our main results and conclusions in Section 6.

\section{Observations}

\subsection{Sample Selection and Stellar Properties}

The CPS has monitored the RVs of more than 2500 stars for as many as 20 years with a typical precision of $\sim 1 \mathrm{~m} \mathrm{~s}^{-1}$, providing both a long time baseline to analyze stellar jitter and high instrumental precision. Our sample is composed of stars observed as part of the CPS with stellar parameters from Brewer et al. (2016, erratum Brewer et al. 2017; cited as B17 hereafter). From this sample of over 1600 stars, we have identified those stars with masses above $0.7 M_{\odot}$. This lower mass limit is mainly a result of the B17 sample, which has a lower temperature limit of $\sim 4700 \mathrm{~K}$. To ensure robust mass measurements, we choose only those stars for which the spectroscopically derived surface gravities agree with the isochrone-derived surface gravities-both given in B17-to within $5 \%$ (J. M. Brewer 2020, private communication). We further narrow this sample down by requiring more than 10 observations from Keck/HIRES to ensure enough measurements to measure RV variability. The 10 observation requirement was chosen in order to properly disentangle center-of-mass motions due to orbital companions from the intrinsic stellar variability (see Section 5.2.2 for more details on this requirement). Our final sample is made up of 617 stars. An $\mathrm{H}-\mathrm{R}$ diagram of our sample is shown in Figure 1. We point out a paucity of stars between $\log g \approx 3.5$ and 3.8, which has two possible causes. First, an observational bias: the original CPS sample targeted mostly main-sequence stars before a large number of subgiants and giants were added to the sample as part of the "retired" A-star survey (Johnson et al. 2006). The region of few stars is therefore likely near the boundaries of these two samples: post-main-sequence stars that are at the edges of the "subgiant" region. We note that as the "retired" 


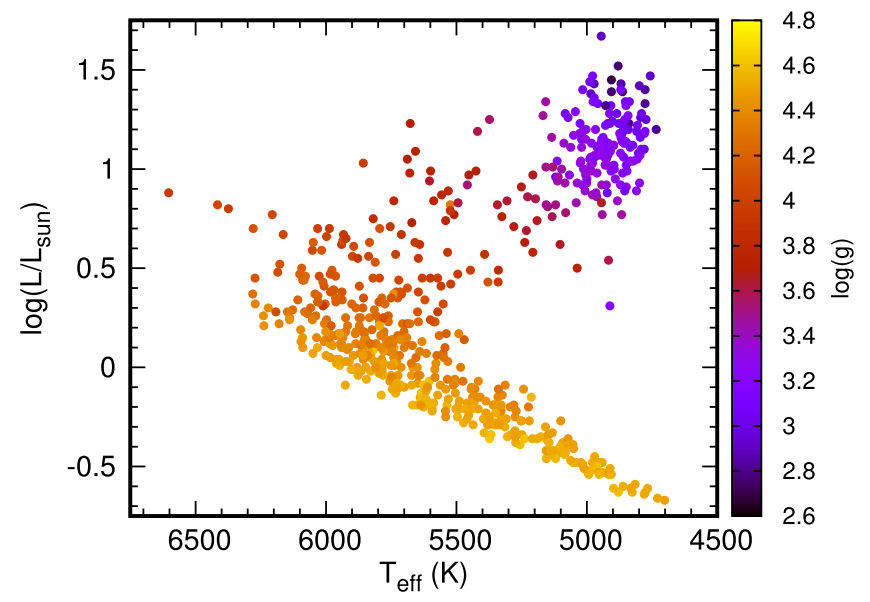

Figure 1. H-R diagram showing our sample. Points are colored by $\log g$ for reference. Note the thinning of stars in our sample between $\log g \approx 3.5$ and 3.8 , which likely comes from the short evolutionary time stars spend in this regime. However, it could also arise from the selection biases of the CPS and "retired" A-star surveys.

A-star survey specifically targeted intermediate-mass stars, we expect our sample of evolved stars to be biased toward those masses $\left(\geqslant 1.1 M_{\odot}\right)$.

The second, and likely more important, effect is astrophysical: stars simply spend a very small portion of their lives in this region of the H-R diagram, zipping through it in relatively no time at all compared to their main-sequence or even giant-branch lifetimes (Kippenhahn et al. 2012). For more-massive stars (several solar masses), this corresponds to the classical Hertzsprung gap, where there is a very low chance of observing stars. Because our sample is lower mass, these stars lie on the bottom edge of the Hertzsprung gap, and we therefore expect from the outset to see only a few stars here. The stars in our sample range in mass from $0.7 M_{\odot} \leqslant$ $M_{\star} \leqslant 2.14 M_{\odot}$, effective temperatures from $4702 \mathrm{~K} \leqslant T_{\text {eff }} \leqslant$ $6603 \mathrm{~K}$, and surface gravities from $2.70 \leqslant \log g \leqslant 4.69$.

Central to this work is a set of reliably measured stellar parameters. We choose those from B17, who used onedimensional LTE model spectra to fit a star's observed spectrum to determine effective temperatures, metallicities, surface gravities, and elemental abundances. In conjunction with Hipparcos parallaxes and $V$-band magnitudes, these spectral measurements were used to derive masses, radii, and luminosities. ${ }^{8}$ Their iterative fitting technique and improved line list correct for systematic discrepancies in $\log g$ between spectroscopy and asteroseismology (Huber et al. 2013; Bastien et al. 2014a), and their spectroscopic methods are now consistent with the values of $\log g$ obtained from asteroseismology for stars within the asteroseismic calibration range

\footnotetext{
8 Although Gaia DR2 is now available with updated parallaxes, the effect this has on the stellar properties is minimal as these are generally bright targets with well-measured Hipparcos data. Among our sample, Gaia and Hipparcos distances agree to a median of $3 \%$. The largest offset comes from giant stars ( $\log g<3.5,156$ stars in our sample) that are distant, faint, and cool, where the median difference is $8 \%$. The spectroscopic parameters $\left(T_{\text {eff }}, \log g, v \sin i\right.$, and abundances) calculated in B17 are all independent of the distance. The luminosity is dependent on distance, which affects the mass (and radius, but our analysis does not use radii). In general, we find that the difference tends toward larger distances in the Gaia catalog, and therefore, stars are more luminous than in Hipparcos. The effect is that for these stars, the median $8 \%$ distance error leads to an observed $16 \%$ luminosity error and subsequently an expected $16 \%$ mass error (given the fixed $\log g$ ).
}

(Brewer et al. 2015). Outside of this calibration range, Brewer et al. (2015) have taken great care to show that they see no systematic trends with effective temperature or metallicity that had been seen in previous work (Valenti \& Fischer 2005). Specifically for low-mass stars, where asteroseismic data are unavailable, comparisons were made with a sample of stars with gravity constraints from transiting exoplanets.

A key portion of our analysis is an investigation of RV jitter trends by mass. There has been ongoing debate surrounding the reported masses of the intermediate- mass subgiants targeted by RV surveys such as the "retired" A-star survey (Johnson et al. 2006). For a detailed summary of this debate, see Stello et al. (2017) and references therein. Indeed, Stello et al. (2017) measured asteroseismic masses for eight stars and found that planet discoveries appear to have systematically overestimated the masses of stars above $1.6 M_{\odot}$. Despite this worrisome conclusion, given the good agreement of the surface gravities from B17 with asteroseismology, we expect that the derived masses should also agree with asteroseismically derived masses. Finally, more recent work by Ghezzi et al. (2018) show that despite a small overestimate in evolved star masses, it is not nearly as large as the $50 \%$ overestimate as suggested by Lloyd (2011, 2013).

\subsection{Spectra and RV Measurements}

Observations were taken at Keck Observatory using the HIRES with resolution $R \approx 55,000$. The CPS employs a standard observing procedure for bright stars that ensures uniform signal-to-noise ratio and instrumental/algorithmic velocity precision on all bright FGK targets. Typical values for a $V=8$ magnitude star is a signal-to-noise ratio of 190 at $5800 \AA$ for an exposure of $90 \mathrm{~s}$. RVs are calculated using the iodine-cell calibration technique and the forward-modeling procedure described in Butler et al. (1996) and later Howard et al. (2011). Our velocity data set is largely the same as the sample in Butler et al. (2017), although the RVs were calculated using a different pipeline.

For several stars that were known planet hosts, we included in the planet-fitting procedure non-Keck RV measurements as published with the initial discovery or in the most recent orbital analysis. Table 1 lists the stars for which we included additional velocities as well as the telescopes and spectrographs where the measurements were taken. We mainly use previously published RVs only for the stars for which we are unable to reproduce the published planetary models with Keck data alone during our vetting process, and so Table 1 is therefore not an exhaustive list of all previously published velocities for all 617 stars in this sample.

\subsection{The Activity Metric}

The primary activity metric used in this work is the Mount Wilson $S$ index, $S_{\mathrm{HK}}$, which measures the emission in the cores of the $\mathrm{Ca}$ II $\mathrm{H} \& \mathrm{~K}$ lines relative to the nearby continuum (Duncan et al. 1991). When a star experiences increased magnetic activity, the flux in the cores of these lines measurably increases. Because the cores of these lines are formed in the chromosphere, $S_{\mathrm{HK}}$ measures the amount of chromospheric emission and is a well-studied index of chromospheric activity of a star.

The wavelength coverage of Keck-HIRES contains the Ca II $\mathrm{H} \& \mathrm{~K}$ lines. As a result, we benefit from having measurements 
Table 1

Summary of Additional Non-Keck-HIRES Velocities

\begin{tabular}{|c|c|c|c|c|}
\hline Star & Telescope & Instrument & $N_{\text {obs }}$ & Reference \\
\hline HD 1502 & Harlan J. Smith Telescope & TCS & 25 & Johnson et al. (2011) \\
\hline HD 1502 & Hobby-Eberly Telescope & HRS & 20 & Johnson et al. (2011) \\
\hline HD 159868 & Anglo-Australian Telescope & UCLES & 47 & Wittenmyer et al. (2012) \\
\hline HD 192699 & Lick Observatory & Hamilton spectrometer & 34 & Johnson et al. (2007b) \\
\hline HD 114613 & Anglo-Australian Telescope & UCLES & 223 & Wittenmyer et al. (2014) \\
\hline HD 38801 & Subaru & High Dispersion Spectrograph & 11 & Harakawa et al. (2010) \\
\hline HD 181342 & CTIAO $1.5 \mathrm{~m}$ & CHIRON & 11 & Jones et al. (2016) \\
\hline HD 181342 & CTIAO $2.2 \mathrm{~m}$ & FEROS & 20 & Jones et al. (2016) \\
\hline HD 181342 & Anglo-Australian Telescope & UCLES & 5 & Wittenmyer et al. (2011) \\
\hline HD 5608 & OAO $1.88 \mathrm{~m}$ & HIDES & 43 & Sato et al. (2012) \\
\hline HD 10697 & Harlan J. Smith Telescope & TCS & 32 & Wittenmyer et al. (2009) \\
\hline HD 10697 & Hobby-Eberly Telescope & HRS & 40 & Wittenmyer et al. (2009) \\
\hline HD 210702 & Lick Observatory & Hamilton spectrometer & 29 & Johnson et al. (2007a) \\
\hline HD 210702 & OAO $1.88 \mathrm{~m}$ & HIDES & 36 & Sato et al. (2012) \\
\hline HD 214823 & Observatoire de Haute-Provence $1.93 \mathrm{~m}$ & SOPHIE & 13 & Díaz et al. (2016) \\
\hline HD 214823 & Observatoire de Haute-Provence $1.93 \mathrm{~m}$ & SOPHIE + & 11 & Díaz et al. (2016) \\
\hline HD 12484 & Observatoire de Haute-Provence $1.93 \mathrm{~m}$ & SOPHIE & 65 & Hébrard et al. (2016) \\
\hline HD 150706 & Observatoire de Haute-Provence $1.93 \mathrm{~m}$ & ELODIE & 48 & Boisse et al. (2012) \\
\hline HD 150706 & Observatoire de Haute-Provence $1.93 \mathrm{~m}$ & SOPHIE & 53 & Boisse et al. (2012) \\
\hline HD 16702 & Observatoire de Haute-Provence $1.93 \mathrm{~m}$ & ELODIE & 22 & Díaz et al. (2012) \\
\hline HD 26965 & La Silla Observatory $3.6 \mathrm{~m}$ ESO telescope & HARPS & 229 & Díaz et al. (2018) \\
\hline HD 28185 & Hobby-Eberly Telescope & HRS & 34 & Wittenmyer et al. (2009) \\
\hline HD 28185 & $6.5 \mathrm{~m}$ Magellan II telescope & MIKE & 15 & Minniti et al. (2009) \\
\hline HD 28185 & $1.2 \mathrm{~m}$ Leonhard Euler Telescope & CORALIE & 40 & Santos et al. (2001) \\
\hline HD 45652 & Observatoire de Haute-Provence $1.93 \mathrm{~m}$ & ELODIE & 14 & Santos et al. (2008) \\
\hline HD 45652 & $1.2 \mathrm{~m}$ Leonhard Euler Telescope & CORALIE & 19 & Santos et al. (2008) \\
\hline HD 45652 & Observatoire de Haute-Provence $1.93 \mathrm{~m}$ & SOPHIE & 12 & Santos et al. (2008) \\
\hline HD 47186 & La Silla Observatory $3.6 \mathrm{~m}$ ESO telescope & HARPS & 66 & Bouchy et al. (2009) \\
\hline HD 9446 & Observatoire de Haute-Provence $1.93 \mathrm{~m}$ & SOPHIE & 79 & Hébrard et al. (2010) \\
\hline HD 95128 & Lick Observatory & Hamilton spectrometer & 208 & Gregory \& Fischer (2010) \\
\hline HD 125612 & La Silla Observatory $3.6 \mathrm{~m}$ ESO telescope & HARPS & 58 & Lo Curto et al. (2010) \\
\hline HD 142091 & Lick Observatory & Hamilton spectrometer & 46 & Johnson et al. (2008) \\
\hline HD 1605 & Subaru & High Dispersion Spectrograph & 14 & Harakawa et al. (2015) \\
\hline HD 1605 & OAO $1.88 \mathrm{~m}$ & HIDES & 61 & Harakawa et al. (2015) \\
\hline HD 1666 & Subaru & High Dispersion Spectrograph & 11 & Harakawa et al. (2015) \\
\hline HD 1666 & OAO $1.88 \mathrm{~m}$ & HIDES & 67 & Harakawa et al. (2015) \\
\hline
\end{tabular}

of $S_{\mathrm{HK}}$ made simultaneously with each RV observation ${ }^{9}$ using the method outlined in Isaacson \& Fischer (2010). The $S_{\mathrm{HK}}$ value we report for each star is simply the median of the $S_{\mathrm{HK}}$ time series. Further, by examining the $S_{\mathrm{HK}}$ time series, we can more closely investigate correlations between RV and activity. In particular, given the typical cadence of observations, we can identify stars with activity cycles, which can be especially pernicious for planet hunters as they can induce planet-like RV variations. For this work, we wish to retain the RV signals due to activity cycles as they are intrinsic stellar variability, and it is therefore crucial to identify stars with apparent activity cycles to avoid subtracting out the stellar signal we seek by mistaking it for center-of-mass motion. We follow a similar philosophy for stars with evidence of activity signals with periods similar to possible rotation periods. However, these are generally tougher to identify given the sampling of observations for a typical star in our sample.

We also briefly examined the $\log R_{\mathrm{HK}}^{\prime}$ values for these stars, also from B17. This activity metric accounts for the different continuum levels near the $\mathrm{Ca}$ II lines for different spectral types

\footnotetext{
9 After the $2004 \mathrm{Keck} / \mathrm{HIRES}$ upgrade. There is an offset between the preand post-upgrade $S_{\mathrm{HK}}$ values that is different for each star. Because the majority of the observations in this sample come post-upgrade, we opt to use solely those values. See Section 3.5 for more details on the upgrade.
}

as well as the base photospheric contribution (Noyes et al. 1984). However, it has not been calibrated for subgiant and giant stars, and is therefore only a useful metric for mainsequence stars. Because a large portion of this work investigates the relation between RV jitter and the evolutionary stage of the star, we mostly ignore $\log R_{\mathrm{HK}}^{\prime}$ as an activity metric and instead use $S_{\mathrm{HK}}$. Values of $\log R_{\mathrm{HK}}^{\prime}$ were mainly used in conjunction with $S_{\mathrm{HK}}$ when vetting jitter measurements (see Section 3.2) because of known relations between $\log R_{\mathrm{HK}}^{\prime}$, activity, and RV jitter (e.g., Wright 2005).

\section{Calculating RV Jitter}

Our primary goal in this work is to study the RV variations induced by intrinsic stellar variability. As such, it is crucial that we remove any Keplerian signal caused by a companion that may be present in the data ("planetary noise"). To ensure that the reported RV jitter is in fact due to intrinsic variability and not other effects (companions, instrument errors, etc.), we found it necessary to undergo a vetting process on a star by star basis to remove any non-intrinsic variations, as described in detail in this section (see Appendix B for details on individual systems). Therefore, each star with a jitter measurement here has cleared our vetting process and represents what we interpret to be astrophysical stellar jitter. Only a few stars in our initial 
sample contained RVs that led us to remove the stars from our sample altogether (see Appendix B.12 for a description of these stars). Because we expect that our corrections to the RV time series (subtracting a planet or long-term linear trend, etc.) will not have captured all center-of mass motions that we wish to subtract off (i.e., there are as yet unidentified planets or simply poorly constrained planets contributing noise to our measurements), we certainly have not isolated the true stellar jitter for all of our stars, and rather, the measured jitter presented here represents our best estimate of the true astrophysical stellar jitter.

All Keplerian fits to RV data in this work were done using the IDL RVLIN package (Wright \& Howard 2009), which is capable of performing multiplanet fits as well as incorporating $\mathrm{RV}$ data from multiple telescopes and solving for telescope offsets. Much of the vetting process involves subjective decisions to choose whether or not we believe a Keplerian fit. We do not impose an objective criterion (such as a $\chi^{2}$ goodness of fit cutoff or false-alarm probability of periodogram peaks), as we find that no one criterion can adequately establish whether the fit is indeed due to an orbital companion. We instead use several metrics (both statistical and astrophysical) in conjunction with each other to holistically judge if the fit from RVLIN could indeed be due to an orbital companion.

We are deliberately conservative when deciding if $\mathrm{RV}$ variations are due to companions. In all cases where we subtract the best-fit RV signal of a companion, we list that companion and the best-fit orbital parameters in Table 2. Our conservative approach to subtracting companions means many stars will have an RV "jitter" value here that is inflated by orbital companions that we did not deem sufficiently securely detected to remove. As a result, it is the jitter floor that we have robustly identified, and many stars lying above this floor may in reality be low-jitter stars with as yet unannounced planetary systems that are inflating the measured jitter.

Our procedure for judging a fit is roughly as follows. First, we perform the by-eye test. We examine both the time-series fit and the phase-folded fit to each star. The reduced $\chi^{2}$ value gives us a numerical value on which to anchor our judgment. However, the reduced $\chi^{2}$ can be severely affected by the number of data points as well as systematic errors and can be convincingly low in cases where the best-fit orbit actually traces a stellar activity cycle. For this reason, we also look at both the times series of the activity metric $S_{\mathrm{HK}}$, described in Section 2.3, and the median of the time series. As more active stars are expected to have larger $\mathrm{RV}$ variations, we are generally more suspicious of active stars with planet fits. We also look at the $\log g$ of the star. From Bastien et al. (2014b) and Kjeldsen \& Bedding (1995) and their follow-up, Kjeldsen \& Bedding (2011; also Wright 2005; Dumusque et al. 2011), we expect RV jitter to increase as stars evolve during the subgiant phase. Finally, we look at the resulting RV jitter from the fit. Using our experience and intuition, we can piece all of these elements together to decide whether or not we believe a planetary fit. Again, we generally only subtract a companion if all evidence suggests the RV variations are due to center-ofmass Keplerian motion.

\subsection{Removing Known Planets}

We first take our sample and search for known planet hosts. For each planet-hosting star, we start with the published best-fit parameters for each planet. These serve as the initial parameters that we input to RVLIN, which calculates new orbital parameters. We choose not to simply subtract a Keplerian with the published best-fit orbital parameters because previously undetected planets can change the best-fit planet model or because we have additional RV observations taken after initial publication and so we expect our new best-fit results to be slightly improved. In the majority of cases, we do not find a large change in orbital parameters, although there are a few that now have better-constrained periods, especially for long-period companions.

Once RVLIN has produced a best fit for a system with a known planet or planets, we investigate the phase curves of each planet and examine the residuals (and their periodogram) after subtracting all planets from the system. If we feel based on the periodogram that there is a chance that an additional unpublished planet remains in the data, we revise the fit, adding a period guess from the periodogram for that planet. By comparing the $\chi^{2}$ goodness of fit, phase curves, and resulting RV jitter, we approve or disfavor the extra planets as needed. Note that in 15 cases our analysis revealed previously unpublished planets around subgiants (Luhn et al. 2019), several of which were additional companions to known planet hosts.

For planets that were not discovered with Keck, we sometimes do not have enough observations from the Keck data alone to detect the planet's signal. In these cases, we combine the RVs in the initial discovery paper and the Keck RVs to determine the best-fit planet model (see stars listed in Table 1). In our final calculation of the RV jitter, we ensure consistency by only calculating jitter using the Keck velocities.

\subsubsection{Transiting Planets}

For stars with known transiting planets, we use the transit time and period as fixed inputs to RVLIN and allow it to find the remaining best-fit orbital parameters. Because the planet is known to transit, the star must have an embedded RV signal from that planet. In many cases where the planets-and therefore the semiamplitudes - are small, the fit from RVLIN would not be convincing by our procedure defined above. However, in these cases, the RV jitter is of order the RV semiamplitude, and so subtracting out the signal only affects the resulting RV jitter by a few percent. In these cases, it is likely that we are not subtracting out the true RV signal from the transiting planet; however, because there must be a signal in the data, the only signal we can subtract is the best fit, regardless of how well it appears to fit. The fact that the RV jitter is not largely affected in these cases means that our decision to subtract the signal most likely does not matter, but we subtract anyway for completeness.

For transiting planets, we also inspect velocities near the time of transit for any possible Rossiter-McLaughlin effects (Gaudi \& Winn 2007) during transit. In only one case, HD 189733 , is a clear RM signal present. In this case, we remove the velocities taken during transit so that they do not artificially inflate the measured RV jitter.

\subsection{Blind Fits}

For all other stars, we perform a blind single-planet fit using RVLIN. We do this to account for any planetary signals that may be present in the data, but have so far been missed by planet hunters and have not yet been published. We then follow 
Table 2

Orbital Parameters of Keplerian Signals Subtracted from RV Time Series

\begin{tabular}{|c|c|c|c|c|c|c|c|c|c|c|c|}
\hline Name & Com & $\begin{array}{c}m \sin i \\
\left(M_{\mathrm{Jup}}\right)\end{array}$ & $\begin{array}{c}P \\
\text { (days) }\end{array}$ & $\begin{array}{c}a \\
(\mathrm{au})\end{array}$ & $\begin{array}{c}T_{\mathrm{p}} \\
(\mathrm{JD})\end{array}$ & $e$ & $\begin{array}{c}\omega \\
(\mathrm{deg})\end{array}$ & $\begin{array}{c}K \\
\left(\mathrm{~m} \mathrm{~s}^{-1}\right)\end{array}$ & $\begin{array}{c}\gamma \\
\left(\mathrm{m} \mathrm{s}^{-1}\right)\end{array}$ & $\begin{array}{c}d v d t \\
\left(\mathrm{~m} \mathrm{~s}^{-1}\right)\end{array}$ & Orbit Reference \\
\hline HD 1388 & * & 28.179 & 9941.964 & 9.31 & 2448143.72 & 0.565 & 115.4 & 300.01 & -153.33 & 0 & This work \\
\hline HD 1461 & $\mathrm{~b}$ & 0.028 & 5.772 & 0.06 & 2450366.22 & 0.229 & 26.4 & 3.11 & -2.03 & 0 & Rivera et al. (2010) \\
\hline$\ldots$ & $\mathrm{c}$ & 0.033 & 13.508 & 0.11 & 2439940.70 & 0.477 & 204.4 & 3.10 & 0.00 & 0 & Díaz et al. (2016) \\
\hline HD 4208 & $\mathrm{~b}$ & 0.823 & 828.000 & 1.67 & 2451040.00 & 0.052 & 339.8 & 19.12 & -4.65 & 0 & Butler et al. (2006) \\
\hline HD 4203 & $\mathrm{~b}$ & 1.774 & 437.128 & 1.16 & 2451913.88 & 0.519 & 331.1 & 52.10 & 13.44 & 0.00656 & Kane et al. (2014) \\
\hline$\cdots$ & $\mathrm{c}$ & 3.831 & 8865.852 & 8.65 & 2455823.98 & 0.075 & 175.3 & 35.33 & 0.00 & 0 & Kane et al. (2014) \\
\hline HD 4628 & $\mathrm{~b}$ & 0.016 & 14.728 & 0.11 & 2455764.15 & 0.403 & 313.3 & 1.72 & -1.55 & 0 & This work \\
\hline HD 4747 & $*$ & 49.359 & 12077.336 & 9.64 & 2438393.51 & 0.730 & 266.8 & 704.07 & -117.86 & 0 & Crepp et al. (2016) \\
\hline HD 6558 & * & 17.393 & 7938.025 & 8.37 & 2451362.08 & 0.210 & 43.3 & 155.65 & 35.44 & 0 & This work \\
\hline HD 8574 & $\mathrm{~b}$ & 1.688 & 226.696 & 0.77 & 2453974.90 & 0.351 & 17.3 & 54.05 & -10.75 & 0 & Wittenmyer et al. (2009) \\
\hline
\end{tabular}

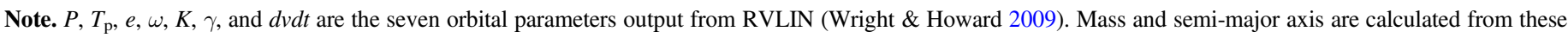
parameters using the stellar mass. Orbit reference lists the reference where the initial guesses to the fitting procedure were obtained.

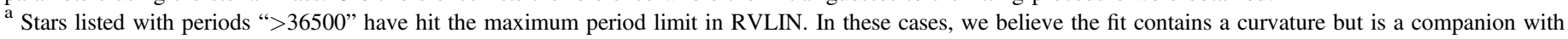
period more than $100 \mathrm{yr}$. We include the fit because we believe it to be subtracting center-of-mass motions, despite a poorly fit and poorly constrained period.

(This table is available in its entirety in machine-readable form.)

up each fit and nonfit with our vetting process to ensure we are left with the most accurate stellar jitter possible. Most blind fits by this method result in the rejection of a planet signal via our vetting process (338 out of 391). In many cases, we were able to quickly discard the fits as spurious because they end up in portions of parameter space where false positives to blind Keplerian fits are common (i.e., fits with $e>0.9$ where the fitter "chases" a single outlying point). However, some require more careful analysis. In the end, our approach was to only accept those fits that have coherent periodic signals that seem to demand subtraction.

The other common result of the blind fit is to find spectroscopic binaries. Because many of these do not have cataloged orbital parameters (often because the period of the system is so much longer than the span of the observations), this often means we are rediscovering these binaries. Luckily, we are not in danger of missing these types of systems as the individual measurement uncertainty is orders of magnitude smaller than the observed RV variations. These systems are usually obvious by eye as having $\sim \mathrm{km} \mathrm{s}^{-1}$ variations, unlike planets, which can be difficult to disentangle from RV jitter in many cases, especially if the observations are spaced out over several years.

In several cases, our blind fit failed to converge on a best-fit solution, but the RV time series and the periodogram showed evidence for potential long-period trends or even sinusoidal variations. In these cases (and even some shorter period cases), we tried another blind fit but with an initial period guess to help RVLIN converge on a fit. The vetting process was then repeated as needed until we obtained a satisfactory fit or were convinced by a lack of fit.

In all, we reemphasize that each star has been through our by-eye vetting process and has been manually confirmed, with many stars being visually inspected two to three times before we were able to conclusively rule out or accept a planet fit and definitively calculate RV jitter. Our sample therefore represents the most comprehensive set of RV jitter measurements from CPS data to date.

Because of our stringent requirements for believing blind fits, the values of RV jitter presented in this work represent upper limits. It is likely (and expected) that many of the stars in our sample still have yet-unsubtracted orbital companions present in the RV time series.

\subsection{Summary of Companion Subtraction Procedure}

In total, we have subtracted 335 companions from 267 stars. Of these 267 stars, 145 were known previously to host planets, leaving 121 "new" systems, many of which are stellar companions. All subtracted companions are listed in Table 2 with the final best-fit parameters that were used in the subtraction, as well as the reference for the orbital parameters used as initial guesses in the fitting procedure, if applicable. We again emphasize that we do not claim that every new planet subtracted is a confirmed planet, rather that we have simply subtracted every strong Keplerian signal that appears to be due to a companion and many are long-period stellar binaries. Because we are focused on the astrophysical interpretation of $\mathrm{RV}$ jitter, a rigorous investigation into the veracity of any planet-mass companions is beyond the scope of this work. Many of the new planets and stellar companions around subgiant stars have been analyzed in more detail in Luhn et al. (2019).

\subsection{Jitter Calculation and Jitter Error}

Our calculation of RV jitter is a simple rms calculation. Once we have obtained our best-fit model to subtract from the velocities, we are left with the residuals,

$$
\epsilon_{i}=\mathrm{RV}_{i}-\mathrm{RV}_{\mathrm{fit}}\left(t_{i}\right)
$$

In the case where no best-fit model was found or the best-fit model was rejected, the residuals used in Equation (1) were simply the unaltered velocities, $\epsilon_{i}=\mathrm{RV}_{i}$. The RV jitter is then simply

$$
j \equiv \sigma_{\mathrm{RV}}=\sqrt{\frac{1}{(N-1)} \sum\left(\epsilon_{i}-\bar{\epsilon}\right)^{2}},
$$

where $N$ is the total number of velocities for the given star. We note that this is not a true rms in the strict sense and is instead a standard deviation uncertainty calculation. Because the zero point for each RV time series is arbitrary, it is necessary to 
subtract off the mean rather than simply taking the square root of the sum of the squared residuals. In the cases where we have accepted a Keplerian fit and have a large number of observations, Equation (2) is essentially the same as an rms because the mean of a $\chi^{2}$ fit is defined to be 0 . In fact, in our sample, the rms and standard deviation agree with the median absolute difference of $0.15 \mathrm{~m} \mathrm{~s}^{-1}$ and mean $0.44 \mathrm{~m} \mathrm{~s}^{-1}$. We continue to refer to $\sigma_{\mathrm{RV}}$ as an "rms." In past works, notably Wright (2005), the "jitter" is found by subtracting the mean reported instrumental uncertainty, $\sigma_{\text {instr }}$, from the rms term $\left(\sigma_{\mathrm{RV}}\right)$ in quadrature. We do not follow that approach in this work because we do not assume to know the instrumental systematics of Keck-HIRES. Subtracting the mean internal error for each star may correctly remove instrumental noise, but it may also introduce or retain systematics that we do not fully understand. Instead, we use the derived RV rms as the reported RV jitter and compare it to the typical Keck-HIRES instrumental uncertainty of $1-2 \mathrm{~m} \mathrm{~s}^{-1}$ (Butler et al. 2017).

Additionally, because this work involves investigating trends with activity, we have many active stars in our sample, which are typically rotating more quickly, leading to broadened absorption features. In principle, this is a concern for measuring precise velocities due to the lack of Doppler content in rapidly rotating stars and should add additional variability to the RV measurements. However, this is incorporated in the reported internal errors, and despite seeing a gradual increase in the median reported single-measurement errors for stars as a function of $v \sin i$, this increase is well below the increase in $\mathrm{RV}$ jitter seen with $v \sin i$, indicating that we have not reached the rotation broadening floor for the stars in our sample.

For our analysis, we also wish to represent the uncertainty in our measurement of the rms. In Section 4, we analyze RV jitter as a function of several stellar properties for our sample of stars. Because each star has a different number of observations, the uncertainty of the measured rms will differ for each star. To that end, we also calculate the error in our measurement of RV jitter and include the calculation in Appendix A. We note simply that the error bars do not account for individual RV measurement uncertainty, the goodness of fit of any subtracted companions, or the potential for the velocities to contain any additional companions.

\subsection{Keck Data before 2004}

In 2004 August, the Keck-HIRES instrument went through upgrades and recommissioning, resulting in an improvement in precision from $4-5 \mathrm{~m} \mathrm{~s}^{-1}$ to $1-2 \mathrm{~m} \mathrm{~s}^{-1}$ in $\mathrm{RV}$ measurements post-2004 (Butler et al. 2017). In several cases, the errors on the RVs before and after this upgrade lead to visually different RV observations (either larger scatter or in some cases RV offsets between pre- and post-2004 observations) and require being treated as observations from two separate telescopes. As mentioned before, RVLIN is capable of separately fitting RVs from multiple telescopes and solving for the offset between them. Because of the sometimes large difference in quality of data between pre- and post-2004 observations, we have taken several approaches to account for this, depending on the individual system's observations.

Removing Pre-2004 Data from Jitter Calculation-In most cases where the pre-and post-2004 data appear different (by comparing the reported errors), we only make use of the pre-2004 data to constrain a fit to the RV data and discard those observations in the final calculation of RV jitter. Our reason for this is the same as discarding other non-Keck observations in our calculation of RV jitter. Because the instrumental errors pre-2004 are significantly higher, they will inherently have larger scatter and will inflate our measurements of RV jitter.

Note that this approach is only taken when we observe noticeable differences between the pre- and post-2004 data. In our sample, only eight stars showed such a necessity (shown in Table 3). For a large number of stars in our sample, we do not observe any obvious differences, and so the pre-and post-2004 data are treated the same and are included in both the fitting and final jitter calculation.

Removing Pre-2004 Data Altogether-In only two cases did we find the need to completely ignore the data before the 2004 upgrades (2 out of 617, HD 1205 and HD 101472). These systems have a large quantity of data after the upgrades such that completely removing the pre-upgrade data does not severely limit the number of observations used in the fit. We are not suspicious of any long-period trends or companions for these stars and so including the pre-2004 data to maintain the long baseline is not necessary.

Pre- and Post-upgrade Offsets-For 19 stars, we noticed that there appeared to be a reduction error when calculating the velocities before and after the upgrade, leading to a slight but noticeable offset in the RVs. By treating the pre- and postupgrade velocities as coming from two separate telescopes, RVLIN is able to solve for the offset, which is typically no more than $15 \mathrm{~m} \mathrm{~s}^{-1}$.

In only one case (HD 50639) have errors in RV extraction produced large $\left(\mathrm{km} \mathrm{s}^{-1}\right)$ offsets between pre- and post-2004 $\mathrm{RV}$ s as a result of only containing a single observation in the immediate 2 years following the upgrade. This occurrence is obvious by eye as a large discontinuity in the otherwise smooth RV curve. In this case, the pre- and post-2004 Keck data are so largely offset that RVLIN cannot solve for the offset and instead finds a long-period, highly eccentric fit that manages to explain the discontinuity in 2004 as the periastron passage. To resolve this, we manually apply a first-order offset of $\sim 1 \mathrm{~km} \mathrm{~s}^{-1}$ before inputting the data into RVLIN as two separate telescopes to find the exact offset that minimizes the $\chi^{2}$

\subsection{Activity Cycles and Correlated Activity}

By examining the $S_{\mathrm{HK}}$ periodograms for every star, we notice activity cycles among many stars, which are listed in Appendix B. ${ }^{10}$

To examine the correlation between RVs and $S_{\mathrm{HK}}$, we use a Pearson correlation coefficient:

$$
r=\frac{\sum\left(S_{\mathrm{HK}}\left(t_{i}\right)-\overline{S_{\mathrm{HK}}}\right)\left(\mathrm{RV}\left(t_{i}\right)-\overline{\mathrm{RV}}\right)}{\sqrt{\sum\left(S_{\mathrm{HK}}\left(t_{i}\right)-\overline{S_{\mathrm{HK}}}\right)^{2}} \sqrt{\sum\left(\mathrm{RV}\left(t_{i}\right)-\overline{\mathrm{RV}}\right)^{2}}},
$$

where RV and $S$ represent the set of velocities and $S$ indices. For strongly correlated variables, the Pearson coefficient is near 1 , and for strong anticorrelation, $r$ is near -1 . The ability to simultaneously extract $S_{\mathrm{HK}}$ and the RV from the same stellar spectrum is what allows this correlation to be measured. For

\footnotetext{
${ }^{10}$ The classification of stars as having "activity cycles" in this work is not rigorous. We refer to stars with periodic activity as those with activity cycles, with the periodicity determined by visually examining the strength of peaks in periodograms of activity.
} 
Table 3

RV Jitter and Analysis

\begin{tabular}{|c|c|c|c|c|c|c|c|c|c|c|c|c|c|c|c|}
\hline $\begin{array}{l}\text { Name } \\
\text { (1) }\end{array}$ & $\begin{array}{c}\text { Jitter } \\
\left(\mathrm{m} \mathrm{s}^{-1}\right) \\
(2)\end{array}$ & $\begin{array}{c}\sigma_{j} \\
\left(\mathrm{~m} \mathrm{~s}^{-1}\right) \\
(3)\end{array}$ & $\begin{array}{c}N_{\text {obs }} \\
\text { (4) }\end{array}$ & $\begin{array}{c}\log g \\
(5)\end{array}$ & $\begin{array}{l}S_{\mathrm{HK}} \\
(6)\end{array}$ & $\begin{array}{l}\text { BFF } \\
\text { (7) }\end{array}$ & (8) & $\begin{array}{l}N_{p, p} \\
(9)\end{array}$ & $\begin{array}{l}N_{p, u} \\
(10)\end{array}$ & $\begin{array}{l}\text { LTF } \\
\text { (11) }\end{array}$ & $\begin{array}{l}\text { Outlier } \\
\text { (12) }\end{array}$ & $\begin{array}{r}\mathrm{rms}_{p u} \\
\text { (13) }\end{array}$ & $\begin{array}{c}\text { Removal } \\
\text { (14) }\end{array}$ & $\begin{array}{l}\text { Offset } \\
(15)\end{array}$ & $\begin{array}{c}\text { Nothing } \\
\text { (16) }\end{array}$ \\
\hline HD 105 & 50.546 & 11.260 & 14 & 4.53 & 0.38 & $\checkmark$ & $\ldots$ & $\ldots$ & $\ldots$ & $\ldots$ & $\ldots$ & $\ldots$ & $\ldots$ & $\ldots$ & $\checkmark$ \\
\hline HD 377 & 53.023 & 5.715 & 64 & 4.46 & 0.38 & $\checkmark$ & $\ldots$ & $\ldots$ & $\ldots$ & $\ldots$ & $\ldots$ & $\ldots$ & $\ldots$ & $\ldots$ & $\checkmark$ \\
\hline HD 691 & 22.473 & 6.326 & 17 & 4.48 & 0.56 & $\checkmark$ & $\ldots$ & $\ldots$ & $\ldots$ & $\cdots$ & $\ldots$ & $\cdots$ & $\ldots$ & $\ldots$ & $\checkmark$ \\
\hline HD 1388 & 5.615 & 0.715 & 51 & 4.32 & 0.16 & $\ldots$ & 1 & 0 & 1 & $\cdots$ & $\ldots$ & $\ldots$ & $\ldots$ & $\checkmark$ & $\ldots$ \\
\hline HD 4203 & 3.320 & 0.259 & 49 & 4.08 & 0.15 & $\ldots$ & 1 & 2 & 0 & $\checkmark$ & $\ldots$ & $\ldots$ & $\ldots$ & $\ldots$ & $\ldots$ \\
\hline HD 4307 & 4.047 & 0.472 & 83 & 4.05 & 0.15 & $\checkmark$ & $\ldots$ & $\ldots$ & $\ldots$ & $\ldots$ & $\ldots$ & $\ldots$ & $\ldots$ & $\ldots$ & $\checkmark$ \\
\hline HD 4628 & 2.589 & 0.192 & 188 & 4.54 & 0.19 & $\ldots$ & 1 & 0 & 1 & $\ldots$ & $\ldots$ & $\ldots$ & $\ldots$ & $\ldots$ & $\ldots$ \\
\hline
\end{tabular}

Note. To save space, we have used the following column header abbreviations. Column (1) lists the star name as given in B17. Column (2) is the calculated RV jitter for the star, and Column (3) is the uncertainty in that calculation as given in Equation (A5). Column (4) is the number of observations used in the jitter calculation. Note that while our criterion is that stars have more than 10 observations, we do not apply this criterion to the actual jitter calculation, where we occasionally remove the observations before the Keck upgrades in 2004 from the jitter calculation. This applies to a total of six stars. Columns (5) and (6) are the surface gravities and activity measure used in Figures 2-7. Column (7) is a flag indicating if a blind fit was applied to the system (Blind Fit Flag). The blind fit flag is used to show the stars for which no initial inputs (i.e., published planets) were used in the fit. This flag does not mean that the blind fit was accepted, merely that a blind fit was attempted. Acceptance or rejection of a blind fit is further indicated with the planet flag PF. Column (8) is a Planet Flag to indicate if we have subtracted a companion from the system. Columns (9) and (10) indicate the number of published planets $\left(N_{p, p}\right)$ and unpublished planets $\left(N_{p, u}\right)$ for each system. Column (11), the Linear Trend Flag, indicates if a linear trend was subtracted. Column (12) indicates if any outliers were removed. Column (13) contains a flag for when only the post-upgrade observations from Keck were used in the rms calculation. Column (14) indicates the systems where the pre-upgrade observations were discarded altogether. Column (15) indicates the systems where the pre- and post-upgrade Keck velocities were treated as separate telescopes with an offset between them. Finally, column (16) is a flag that indicates stars for which no alterations were made to the RVs. Note that because we first try a blind fit to every star without a published planet, this flag is equivalent to having $\mathrm{BFF}=1$ with no other flags checked. We include this column to explicitly indicate stars for which the raw RVs were used to calculate the RV RMS (no subtractions or removals).

(This table is available in its entirety in machine-readable form.)

stars that show $|r|>0.5$, we are particularly suspicious of activity-induced jitter and make a special note of them.

In general, we find that among the stars that show a correlation between the RVs and $S_{\mathrm{HK}}$, the majority show a positive correlation (125 stars) as opposed to a negative correlation (19 stars), as also seen in Lovis et al. (2011). If we interpret the activity index $S_{\mathrm{HK}}$ as a proxy for surface starspots and faculae, it follows that as activity increases, the number of spots and faculae also increases, which leads to suppressed convection in those regions. Because the stellar surface has a net convective blueshift from convective granulation, the suppressed convection results in a redward shift, toward positive RVs, leading to a positive correlation between activity and RV.

However, not all stars with activity cycles show evidence of correlated RVs. Similarly, many stars show RVs that are highly correlated with noncyclical, stochastic activity. Despite most correlated RVs showing a positive correlation with activity, we observe a wide range of features among stars with activity cycles and activity-correlated RVs. That is to say, correlated RVs are not always indicative of a cycle, and cycles are not always indicative of correlated RVs. ${ }^{11}$ The relation between activity and how it manifests in the RVs remains an open question. A detailed discussion of these features is beyond the scope of this paper and for now we describe individual stars in Appendix B.

To summarize, our efforts to subtract companions in order to retain the stellar jitter means that we must ensure that any periodic signals present in the RVs are not in fact due to an activity cycle

\footnotetext{
${ }^{11}$ In the case of a star showing an activity cycle but no correlated RVs, this could be explained by having RVs that are significantly rotationally modulated, such that the rotationally modulated RVs no longer correlate with the overall activity cycle.
}

that is correlated with the RVs. Because stellar astrophysical jitter includes cycles, we have made the effort to examine the correlations between activity and RVs on a star by star basis.

\subsection{Outliers}

In many stars, we see RV observations that appear to be obvious outliers in the data, usually in one of two ways. In the majority of cases, we notice that the reported errors for a given observation are several times larger than the typical errors for that star. Usually this also occurs with velocities themselves that appear to be significantly displaced from the mean. As a general rule, we remove these points if the errors are larger than 2.5 times the typical errors. In other cases where the velocity rather than the error is what identifies it as an outlier, we also investigate the reported $\chi^{2}$ of the fit to the stellar spectrum as reported by the RV measurement pipeline. This indicates observations where the extraction of an RV was more difficult and is not always represented in the velocity error. We describe instances of outlier removal on individual systems in Appendix B.

\subsection{Summary of Total Sample Statistics}

In all, we applied some sort of correction to the RVs (outlier rejection, companion subtraction, etc.) for more than half of our sample, with only 303 of the 617 stars having an RV jitter simply calculated as the rms of the unaltered velocities, highlighting the need for our careful approach. A total of 158 stars had velocities that produced a successful Keplerian fit by RVLIN but did not pass our vetting procedure and resulted in rejected fits (that is, we did not alter the velocities). Table 3 gives the calculated jitter for each star and lists what changes, if 
any, have been made to the raw RVs of each system. Note that our criterion that stars have more than 10 observations is used for judging possible companions. We do not apply this criterion to the actual jitter calculation, where we occasionally remove the observations before the Keck upgrades in 2004 from the jitter calculation. In these cases, the pre-upgrade observations are enough to confirm or reject a planet, but will inflate the measured RV jitter if included in the jitter calculation. This applies to a total of six stars (HD 1388, HD 8765, HD 30708, HD 35974, HD 191876, and HD 216275). Detailed notes on individual systems can be found in Appendix B.

\subsection{Theoretical Calculations of Convective Components of $R V$ Jitter}

The previous sections all dealt with the empirical measurement of jitter for our sample of stars. The following two sections deal with calculating a theoretical RV jitter for the two convective components we account for in this work: stellar oscillations and granulation. The theoretical calculations will later be used to compare with the empirical results.

\subsubsection{Theoretical Oscillation Component of RV Jitter}

Kjeldsen \& Bedding (2011) provide a theoretical scaling relation for the velocity amplitude of p-mode oscillations at $\nu_{\max }$, the frequency at which the oscillation power peaks,

$$
A_{\mathrm{vel}} \propto \frac{L \tau^{0.5}}{M^{1.5} T_{\mathrm{eff}}^{2.25}}
$$

where $\tau$ is the mode lifetime, for which scaling relations have not been solidly established (see discussions in Kjeldsen \& Bedding 2011; Kallinger et al. 2014). For this work, we choose the mode lifetime scaling relation found in Corsaro et al. (2012),

$$
\tau \propto \exp \left(\frac{5777 \mathrm{~K}-T_{\mathrm{eff}}}{T_{0}}\right)
$$

where $T_{0}=601 \mathrm{~K}$. Putting this all together and scaling it to measured solar observations (Kjeldsen \& Bedding 1995), we get

$$
A_{\mathrm{vel}}=0.234 \mathrm{~m} \mathrm{~s}^{-1}\left(\frac{T_{\mathrm{eff}}}{T_{\mathrm{eff}, \odot}}\right)^{1.75}\left(\frac{R}{R_{\odot}}\right)^{-1}\left(\frac{g}{g_{\odot}}\right)^{-1.5} \tau^{0.5}
$$

However, Equation (6) gives the amplitude of p-mode oscillations. To derive a scaling relation for the rms of this velocity, we assume that oscillation manifests as a single sinusoid with $\nu=\nu_{\max }$, whereby the rms is $0.7087 A_{\mathrm{vel}}$, which gives

$$
\sigma_{\mathrm{osc}}=0.166 \mathrm{~m} \mathrm{~s}^{-1}\left(\frac{T_{\mathrm{eff}}}{T_{\mathrm{eff}, \odot}}\right)^{1.75}\left(\frac{R}{R_{\odot}}\right)^{-1}\left(\frac{g}{g_{\odot}}\right)^{-1.5} \tau^{0.5}
$$

While choosing a single sinusoid with amplitude $A_{\text {vel }}$ is a simplistic view of stellar oscillations, it should only affect the scaling constant and should still capture the evolutionary trends we seek to observe across the sample.

\subsubsection{Theoretical Granulation Component of RV Jitter}

The RV jitter due to granulation is a scaling relation that follows from the granulation size and the number of convective cells on the surface of the star. The proportionality comes again from Kjeldsen \& Bedding (2011),

$$
\sigma_{\mathrm{gran}} \propto \frac{H_{\mathrm{p}} c_{\mathrm{s}}}{R} \propto \frac{L^{0.5}}{M T_{\mathrm{eff}}^{0.5}},
$$

where $H_{\mathrm{p}}$ is the pressure scale height, which is the characteristic size of a granular region, and $c_{\mathrm{s}}$ is the sound speed on the surface of the star. These relations assume a constant mean molecular weight, $\mu$. To derive a proper scaling relation, we require a value for the Sun's RV rms due to granulation, a difficult quantity to measure. However, Meunier et al. (2015) derived an expected value of $0.8 \mathrm{~m} \mathrm{~s}^{-1}$ based on simulations of granulation and supergranulation. More recent work by Milbourne et al. (2019) used the HARPS-N spectrograph to continuously observe the Sun as it would appear as a star (described in Dumusque et al. 2015) and found an RV rms of $1.2 \mathrm{~m} \mathrm{~s}^{-1}$ after accounting for the suppression of convective blueshift by bright magnetic regions. Given the general agreement between these two values, we choose to split the difference between the two and adopt a simple value of $1 \mathrm{~m} \mathrm{~s}^{-1}$. Our scaling relation is then

$$
\sigma_{\text {gran }}=1 \mathrm{~m} \mathrm{~s}^{-1}\left(\frac{L}{L_{\odot}}\right)^{0.5}\left(\frac{M}{M_{\odot}}\right)^{-1}\left(\frac{T_{\text {eff }}}{T_{\text {eff }, \odot}}\right)^{-0.5} .
$$

We note that the granulation term in this work does not distinguish between the signal from the three scales of granulation: granulation, mesogranulation, and supergranulation. Each of these has different physical scales $(1,5,30 \mathrm{Mm})$, different flow velocities $\left(1 \mathrm{~km} \mathrm{~s}^{-1}\right.$ vertical flow, $0.06 \mathrm{~km} \mathrm{~s}^{-1}$ vertical flow, $0.4 \mathrm{~km} \mathrm{~s}^{-1}$ horizontal flow), and different lifetimes $(0.2,3,20 \mathrm{hr})$, which make their individual contributions to RV jitter difficult to study (Rast 2003). However, based on the arguments in Kjeldsen \& Bedding (2011) and Meunier \& Lagrange (2019), we expect that these three granulation effects all follow the same scaling relation. Therefore, by roughly scaling it to the solar values, we are merely scaling the magnitude of the combined granulation, mesogranulation, and supergranulation effects, despite the fact that in practice stars will have variability due to each of these effects, which operate on different timescales. This is justified given the observing cadence of planet-search stars, which is too infrequent to resolve these individual components. The total $\mathrm{RV}$ rms due to convection (both granulation and oscillation) is found by summing the two terms (Equations (7) and (9)) in quadrature.

\section{Empirical Analysis of RV Jitter}

After applying our vetting process to our large sample of stars, we can perform our analysis, examining how RV jitter correlates with stellar parameters. We begin by examining bulk trends in the entire sample. However, first we wish to briefly summarize some key findings of previous works that also investigated the CPS stars monitored with Keck-HIRES.

Wright (2005) examined a subsample of CPS stars without known planets and used an activity metric $\Delta F_{\mathrm{Ca} \text { II }}$, which accounts for the minimum activity of stars as a function of $B-V$ (Rutten 1984), finding that RV jitter increases with this activity metric. Importantly, Wright (2005) notes that K- and G-type stars show slightly lower levels of RV jitter. Isaacson \& Fischer (2010) performed a similar analysis but used $\Delta S_{\mathrm{HK}}$ to 
observe trends with activity. One of the key results of this work was that the RV jitter of $\mathrm{K}$ dwarfs showed little dependence on magnetic activity and showed the lowest overall levels of RV jitter, representing a "sweet spot" for exoplanet searches.

Our analysis builds on these previous works in several ways. The first is our thorough approach to calculating RV jitter. Wright (2005) removed known planet hosts from the sample, and Isaacson \& Fischer (2010) did not give special treatment to planet hosts or possible companions. The resulting jitter values certainly contained dynamical velocities, which is why they simply investigated the jitter floor. By accounting for planets and other companions in a consistent, conservative manner, we have brought many of the artificially high points down to the jitter floor, thereby strengthening its significance. Further, we have the benefit of several years' worth of additional observations that give us a better handle on the long-timescale RV variability as well as better constraints on long-period planets and other long-term trends. Tied to this are the years of observations on stars that were not previously in the CPS sample or had very few observations at the time of publication of either Wright (2005) or Isaacson \& Fischer (2010). As mentioned previously, this includes the sample of "retired" A stars (Johnson et al. 2007a) as well as a sample of young, active stars that had been observed as part of the Spitzer Legacy Program, Formation and Evolution of Planetary Systems (Meyer et al. 2006). These two samples are crucial for this work as they give us much stronger leverage on both activity and evolution. Finally, we restrict ourselves to stars with updated stellar properties from B17, which allows us to investigate more clearly how stellar RV jitter manifests for different stellar types in a more precise manner than previously possible.

Figure 2 shows the RV rms of our sample in two different ways. First, it is shown as a function of $\log g$ and colored by their activity, $S_{\mathrm{HK}}$. In this panel, we have cut off the $y$-axis to exclude stars that have large levels of jitter that are likely due to additional companions that cannot fully be constrained and to better show the trends among the low-jitter stars. The second panel shows the same data but plotted as a function of activity, $S_{\mathrm{HK}}$, and color-coded by their evolutionary state, $\log g$.

The first panel in Figure 2 has two immediately noticeable features: a vertical pileup of stars at high surface gravities (log $g \sim 4.5)$ and a horizontal pileup among the low surface gravity stars. By noting the color-coding, it is clear that the vertical pileup contains the active stars and the inactive stars are contained in the horizontal pileup. We can therefore easily see the two regimes of RV jitter-activity-dominated and convection-dominated. The second panel in Figure 2 does not make as clear of a distinction between these two regimes. Instead, we see a general trend where jitter decreases with decreasing activity while stars are still in the main sequence (yellow-shaded points). Upon leaving the main sequence (orange/red points), we see that decreasing activity results in an increase in RV jitter. We note that because we are looking at the full sample spanning several spectral types, a clear relation between $S_{\mathrm{HK}}$ is not expected. Upon closer examination, Figure 2 (in particular the top panel) paints a general astrophysical picture of RV jitter evolution, as follows:

The Main Sequence-The main sequence is easily identified by the vertical pileup on the left of the upper panel of Figure 2, at $\log g \sim 4.5$. These main-sequence stars are further distinguished by the large fraction of active stars $\left(S_{\mathrm{HK}} \gtrsim 0.25\right)$ in this portion of the plot. Given the known correlation between RV jitter and activity (Campbell et al. 1988; Saar et al. 1998;
Santos et al. 2000; Wright 2005; Isaacson \& Fischer 2010), this is unsurprising based on general main-sequence evolution (e.g., Mamajek \& Hillenbrand 2008). We expect that stars start out on the top left of this diagram (at the top of the main sequence here), as rapidly rotating, active stars with high surface gravity characteristic of the zero-age main sequence (ZAMS). As they live out their lives on the main sequence, they lose angular momentum to magnetic winds, spin down, and become less active. As a result, they become quieter in RV observations and are seen to have less jitter. Therefore, a given star's path on the main sequence is to drop vertically as it becomes less active and less jittery. Eventually, as stellar evolution progresses, its surface gravity drops, and it enters the subgiant and giant regime where it tends to be inactive (Wright 2004). A typical surface gravity for a terminal-age main sequence (TAMS) Sunlike star is $\log g \approx 4$. We emphasize that a portion of the inactive horizontal floor seen in the upper panel of Figure 2 is during a star's final main-sequence evolution. Therefore, we find it useful to distinguish between the main sequence (the time in which the star is burning hydrogen in its core) and the phase in which the star is in the vertical portion of Figure 2. We introduce the term "active main sequence" when we wish to refer specifically to the vertical pileup of active stars. Stars on the "active main sequence" are stars whose jitter is dominated by magnetic activity.

Subgiants and Giants-The remainder of a star's life (at least until into the early giant phase, beyond which we cannot probe with this sample) is spent moving mostly horizontally to the right in the upper panel of Figure 2. These stars are no longer active, as they have spun down to the point where they have very weak magnetic fields and therefore little chromospheric emission. However, we see a noticeable increase in RV jitter as stars evolve and their convective power increases. Among giants and subgiants, stars with lower $\log g$ show higher levels of RV jitter than do stars with higher $\log g$, a result expected and seen by Wright (2005), Kjeldsen \& Bedding (2011), and Bastien et al. (2014b). As these are almost entirely inactive stars that have fully spun down, their jitter is dominated by convection through a combination of granulation and oscillations. The increase in RV rms with decreasing $\log g$ can be more easily seen when the $y$-axis is plotted in log scale, as seen in Figure 3, where we now color-code by mass.

We therefore see from Figure 2 that RV jitter tracks stellar evolution as stars transition from active to inactive stars, and then exhibit increased convective power as they continue to evolve. From Figure 3, we see color gradients that indicate strong mass dependencies, namely the lower $\log g$ and higher $\mathrm{RV}$ jitter with increased mass and the increase in RV jitter with mass among the most active stars. To examine these trends more closely, we divide our sample into mass bins.

Figures 4 and 5 are the same as the first panel in Figure 2 (i.e., RV rms versus $\log g$ ), and Figures 6 and 7 are the same as the second panel (i.e., RV rms versus $S_{\mathrm{HK}}$ ) but now they have been broken into mass bins, each $0.1 M_{\odot}$ wide. ${ }^{12}$ For reference,

\footnotetext{
${ }^{12}$ We note that our mass bins are smaller than the typical uncertainty in the mass from B17. The median mass uncertainty in our sample is $0.16 M_{\odot}$ $(\sim 15 \%)$. As such, it is likely that a number of our stars do not actually fall within the mass bin to which we have assigned them. We have settled on bin widths of $0.1 M_{\odot}$ after comparing several different bin widths, and seeing that our conclusions hold for both larger and smaller bin widths. The bin width we used is both convenient numerically while being small enough to track the differences across mass bins but large enough to contain enough stars to clearly show trends within a bin.
} 


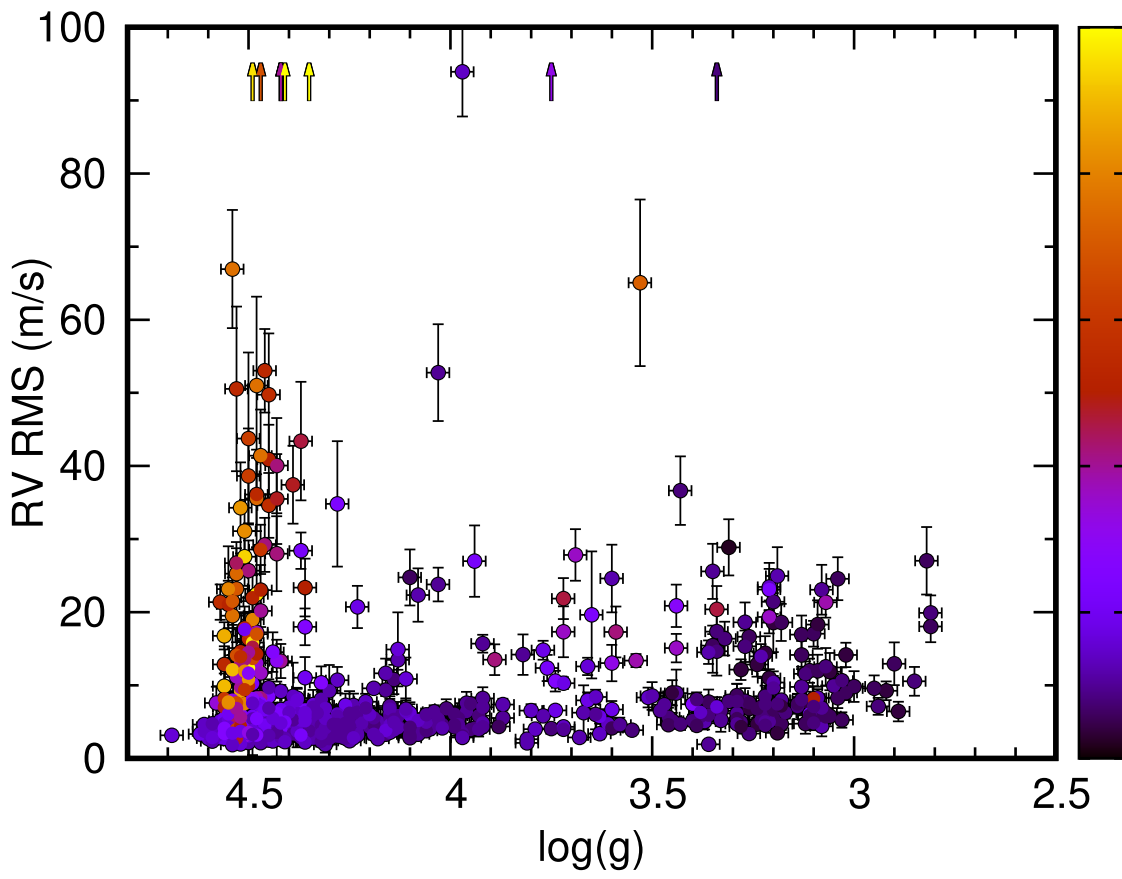

0.6

0.5

0.4

(ึ)

0.3

0.2

0.1

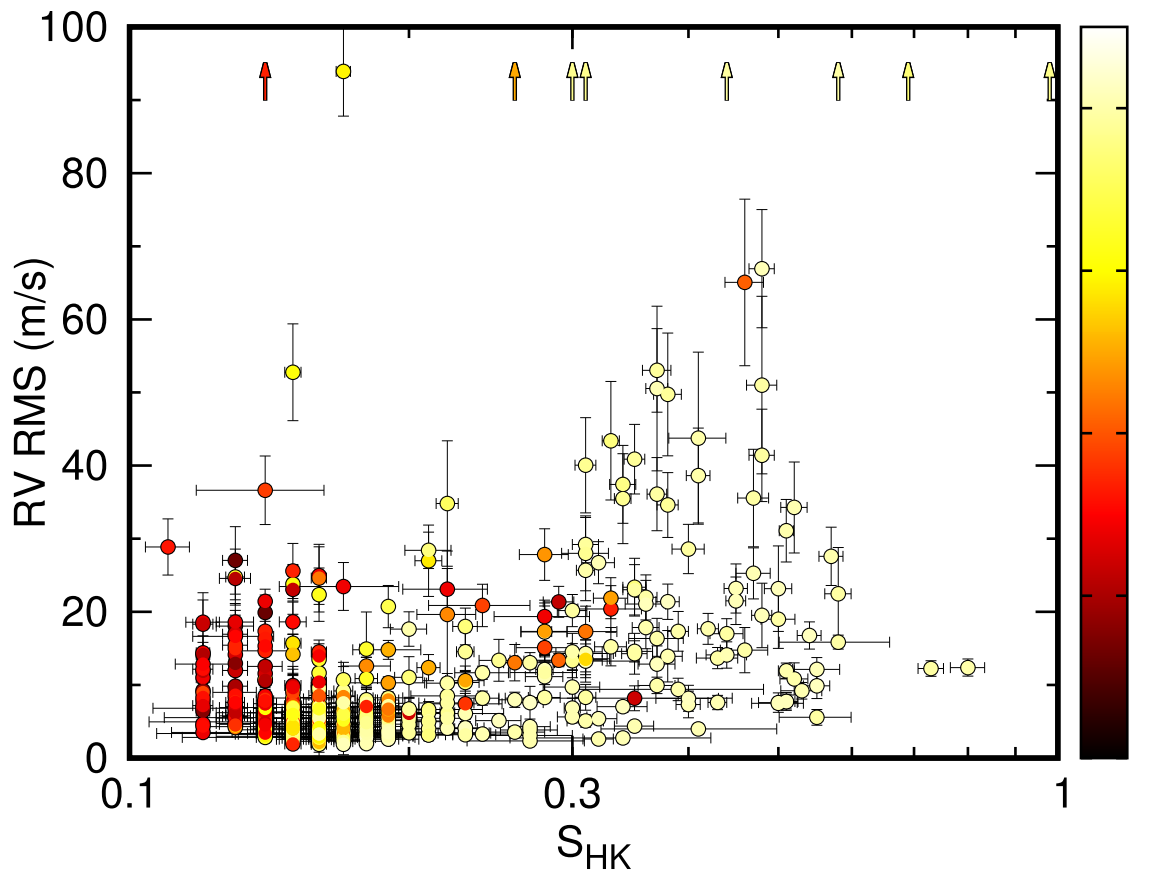

Figure 2. Top: RV jitter as a function of $\log g$ for our entire CPS sample. The $y$-axis has been capped at $100 \mathrm{~m} \mathrm{~s}^{-1}$ to show only those stars whose jitter are likely dominated by astrophysical sources rather than additional unsubtracted companions (upward-pointing arrows indicate the eight stars with RV rms above $100 \mathrm{~m} \mathrm{~s}{ }^{-1}$, two of which overlap in this plot with identical $\log g$ values of 4.42). The vertical error bars are calculated from Equation (A5) as described in Appendix A. The horizontal error bars are uniformly $0.028 \mathrm{dex}$ in $\log g$ as described in B17. The color bar shows the activity metric $S_{\mathrm{HK}}$ as defined in Section 2.3 . As a typical star evolves, it will begin at the top left of this plot with large surface gravity, high activity, and high jitter; it then moves vertically downward due to main-sequence spindown; as it evolves off the main sequence, it transitions from activity-dominated jitter to convection-dominated jitter where the jitter shows a gradual increase with evolution. Bottom: RV rms as a function of $S_{\mathrm{HK}}$ for the stars in our sample. Horizontal error bars come not from individual measurement uncertainties for $S_{\mathrm{HK}}$ but instead are the standard deviation of the $S_{\mathrm{HK}}$ time series, effectively indicating how variable a star is in activity (typically from cycles). Points are colored by their surface gravity. Again, the eight stars with RV rms greater than $100 \mathrm{~m} \mathrm{~s}^{-1}$ are shown as upward arrows. As a star evolves, it will begin on the right side of this plot with high jitter; it then moves diagonally down to the left as it spins down, eventually increasing again as an inactive giant star, when convective phenomena dominate the RV jitter.

we have added the Sun to the plot of stars $1.0 \leqslant M_{\star}<1.1 M_{\odot}$, plotted as a large diamond symbol. ${ }^{13}$

\footnotetext{
${ }^{13}$ We use the measured disk-integrated solar RV rms of $8 \mathrm{~m} \mathrm{~s}^{-1}$ (over the solar cycle) from Meunier et al. (2010). The solar $S_{\mathrm{HK}}$ is 0.1694 averaged over the solar activity cycle (Egeland et al. 2017).
}

For these plots, it is useful to compare trends as they relate to astrophysical checkpoints in their evolution. For that reason, in each mass bin, we plot three vertical lines, which correspond to the surface gravities at three points in the evolution of a star: the ZAMS, the TAMS, and the base of the red giant branch (BRGB). 

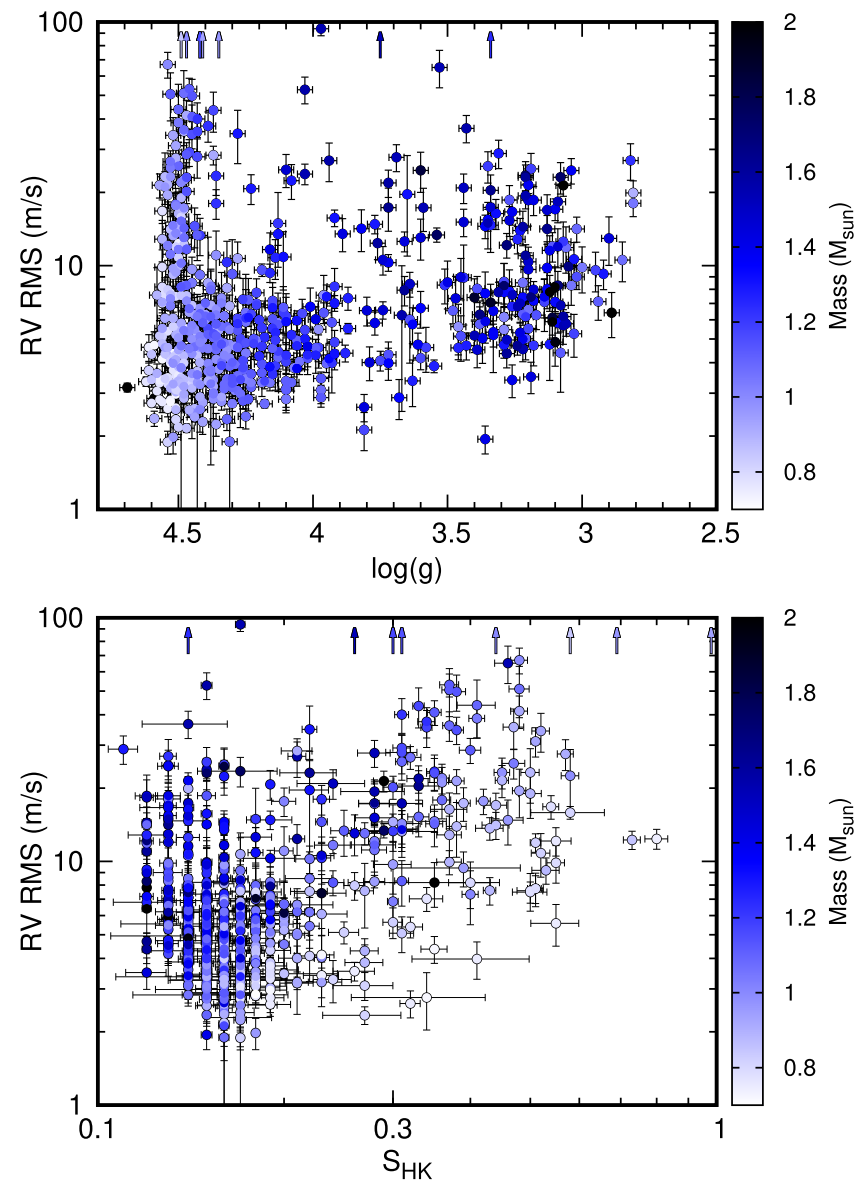

Figure 3. Same as Figure 2, but with the following changes: the $y$-axes have been transformed to log scale and the points in both plots are now color-coded by mass. The mass color-coding highlights two effects: (1) the shift toward lower $\log g$ and higher RV rms with higher mass star in the top panel, and (2) among active stars (above $\sim 0.25$ ), the higher RV jitter associated with higher mass seen in the bottom panel. These plots motivate the analysis by mass bin to see trends more clearly as a function of mass.

These values come from a simple MESA (Paxton et al. 2013) stellar evolution model where we set the TAMS by determining the age at which the hydrogen core fraction falls below $X_{c}=0.0002$ and the BRGB by determining the local minimum on the H-R diagram (van Saders \& Pinsonneault 2013). These three lines break each plot into the three basic phases of evolution that they cover: main sequence, subgiant, and giant phases. The inclusion of the TAMS line also highlights the fact that stars are known to evolve while on the main sequence (Mamajek \& Hillenbrand 2008) and illustrates the effects this has on RV jitter. A star's main-sequence lifetime is spent between the ZAMS and TAMS lines, and we can use these as a quick way to estimate spin-down timescales (as indicated by the decreasing activity and $\mathrm{RV}$ jitter) as they compare to the main-sequence lifetime for a given mass.

Additionally, we have included theoretical curves from relations found in Kjeldsen \& Bedding (2011). These curves plot the expected scaling relation of RV jitter with $\log g$ due to the two components of convectively driven RV jitter: stellar oscillations and stellar granulation, as discussed previously in Sections 3.9.1 and 3.9.2 and given in Equations (7) and (9), respectively. To plot them as a function of $\log g$, we use MESA to generate an evolution track for each mass range. For each mass bin, we evolve a solar-metallicity star with the lowest mass in the mass bin from ZAMS to the tip of the red giant branch, because we are only concerned with evolving a star until $\log g=2.5$. We can then use the stellar parameters at each point in the evolution in the theoretical relations, which depend not only on the surface gravity, but also depend on temperature and radius at a given point in the star's evolution. The evolution tracks used in this work can be seen in Figure 8.

\subsection{Two Regimes of $R V$ Jitter: The Transition from Activity- dominated to Convection-dominated}

We have empirically identified two major regimes of RV jitter: magnetic activity-dominated and convection-dominated. As a reminder, we have treated the granulation and oscillation components as one combined regime where the RV jitter is driven by convection and increases with evolution (as opposed to the decrease with evolution seen in the activity-dominated regime). For this sample, we are not as concerned with determining whether a star is granulation-dominated or oscillation-dominated but rather the transition from being activity-dominated to convection-dominated. In fact, based on the theoretical scaling relation, our sample barely contains stars that have evolved enough to probe the transition from granulation-dominated to oscillation-dominated. However, the fact that the floor of the observations seems to increase as theoretically expected, especially in stars with masses 1.2-1.3 $M_{\odot}$, suggests that the oscillation component dominates for stars below $\log g \sim 3$, and we use this as further validation of the scaling relation for the oscillation component. Further, despite good agreement with the data, the theoretical granulation component appears to follow the instrumental uncertainly threshold and so we are reluctant to make strong claims about the relative strengths of $\mathrm{RV}$ variations due to granulation and oscillations. For most stars, we have $\sim 10-30$ observations over a span of years with variable cadence depending on the star, which does not allow us to say much about the physical phenomena with timescales of minutes to hours (granulation, oscillation). It is clear, however, that these theoretical predictions convincingly describe the observations (whereas activity does not as the $S$ value is low despite elevated jitter in some cases). More work will be needed to distinguish different convection-driven processes.

The following paragraphs discuss the transition from activity-dominated to convection-dominated jitter as it depends on mass. First, it is useful to define the "jitter minimum," the point in a star's lifetime where its RV jitter is lowest, which, as our data suggest, occurs at the transition between the activitydominated and convection-dominated regimes.

\subsubsection{Low-mass Stars: $\mathrm{M}_{\star}<0.9 \mathrm{M}_{\odot}$}

For low-mass stars in our sample, we have very few evolved stars due to their long main-sequence lifetimes. As we decrease in stellar mass, the main-sequence lifetime increases and at some point exceeds the age of the universe. All Sun-like stars below a certain mass $\left(\sim 0.9 M_{\odot}\right)$ are therefore activitydominated, and our data suggest that there is a fundamental mass limit where stars have not evolved enough to reach their astrophysical jitter minimum.

The following trends with $\log g$ can be seen in the first two panels of Figure 4. Stars less massive than the Sun are born as young, active stars, and magnetic activity (manifested as spots/ plages/etc.) dominates the RV jitter. As they continue to 

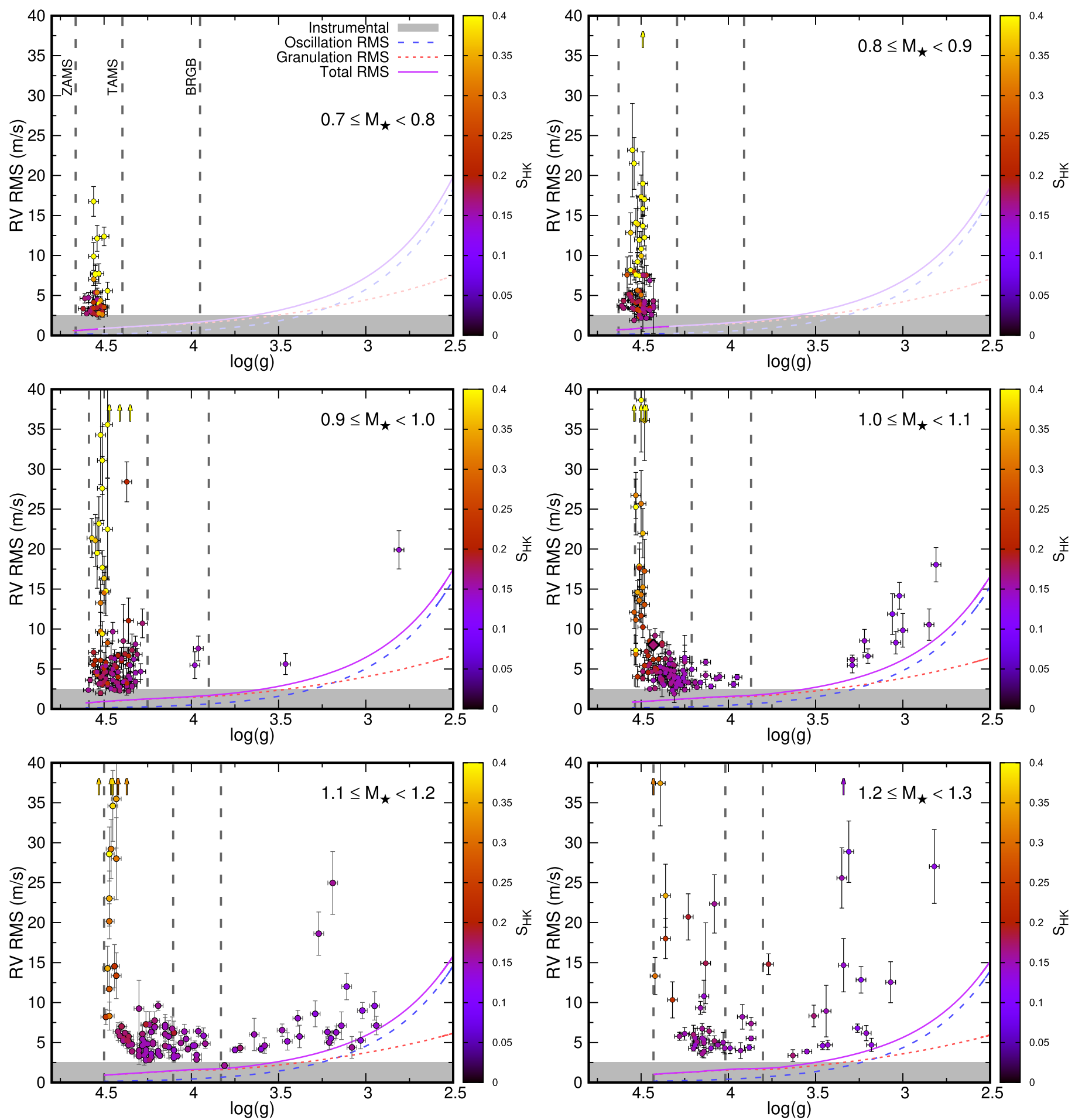

Figure 4. Same as the first panel of Figure 2 but separated by mass. The $y$-axis covers a smaller range to highlight the slope with log $g$. Further, the color scale has been decreased to highlight the transition from the vertical activity-dominated regime to the horizontal convection-dominated regime. Solar values are plotted in the $1.0-1.1 M_{\odot}$ plot as a large diamond $\left(\log g=4.43, \mathrm{RV} \mathrm{rms}=8 \mathrm{~m} \mathrm{~s}^{-1}\right)$. We use the solar rms found in Meunier et al. (2010) and the activity-averaged $S_{\mathrm{HK}}$ from Egeland et al. (2017). The vertical dashed lines plot (from left to right): the theoretical zero-age main sequence (ZAMS) for the lowest mass star; the theoretical terminal-age main sequence (TAMS) for the highest mass star in a given bin; and the base of the red giant branch for the lowest mass in the bin. The shaded region at the bottom of each plot shows the typical Keck-HIRES instrumental uncertainty, which has not been subtracted out from our calculation of RV jitter. The dashed blue and red lines show the RV jitter components for oscillation and granulation, respectively, as given by Equations (7) and (9). Their total contribution to RV jitter (added in quadrature) is shown by the purple line. The theoretical lines in the first two panels have been grayed out for surface gravities where the stellar model is older than the age of the universe. See Figure 5 for the remaining high-mass bins.

evolve on the main sequence, they spin down and become less active, which result in lower RV jitter amplitudes. Because they are still early in their main-sequence lifetimes, they have not changed their structure much and remain near their initial $\log g$.
Their primary movement on a plot of RV jitter versus $\log g$ is therefore vertically downward from their ZAMS location. We note that for most of our mass bins in this regime, we are unable to fully probe the minimum jitter value due to 

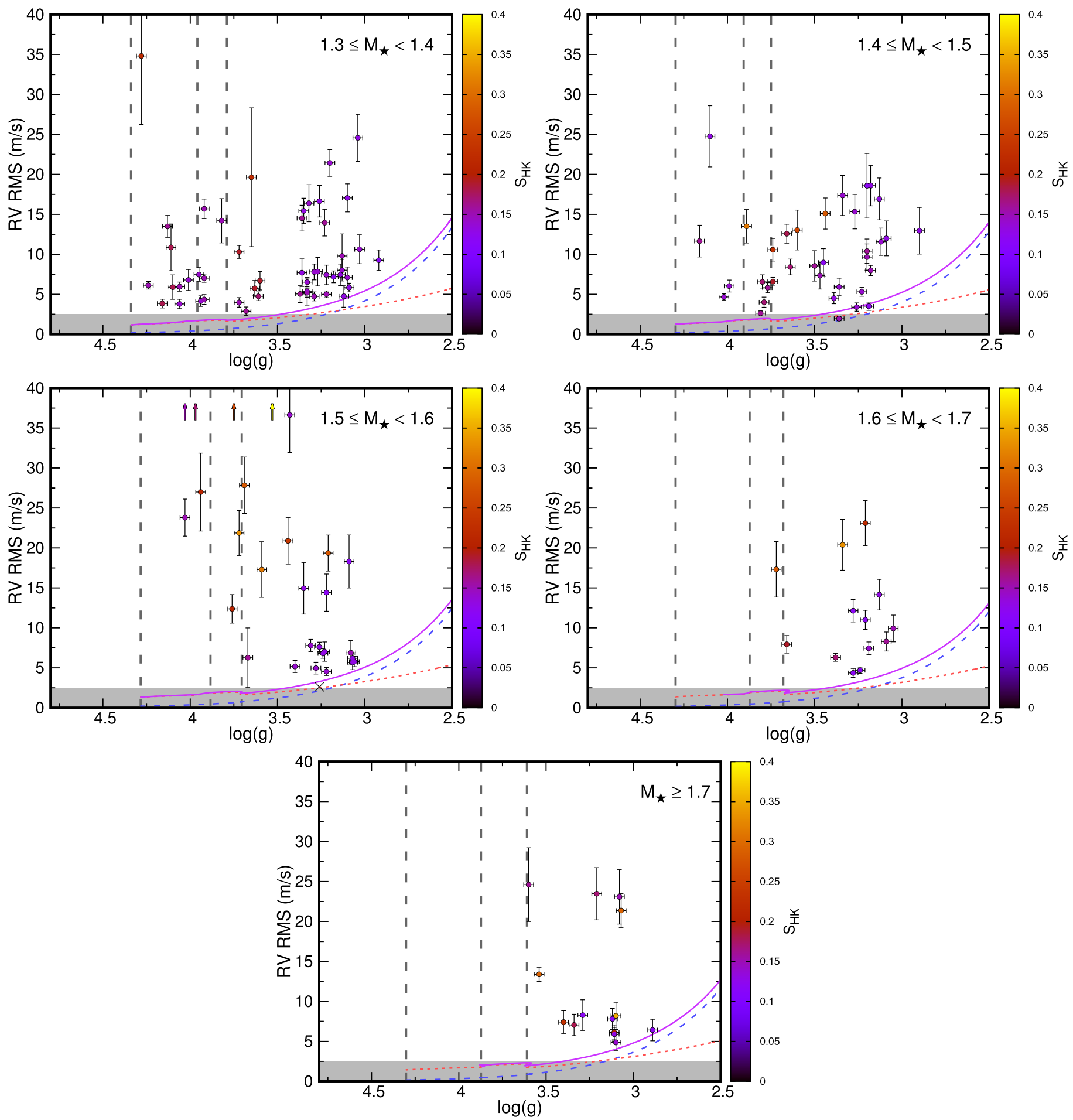

Figure 5. Same as Figure 4, for larger masses. We note the "X" in the 1.5-1.6 $M_{\odot}$ bin, which shows the RV rms of two nights of targeted observations of HD 142091 to observe stellar p-mode oscillations. This serves as a validation of the theoretical scaling relation for the oscillation component (blue dashed line) of the RV rms and is described in more detail in Section 4.2.

instrumental uncertainty. We see some evidence in the stars between 0.9 and $1.0 M_{\odot}$ that the jitter floor for these stars occurs when these stars have begun to evolve toward the end of their main-sequence lifetimes.

When looking at trends with $S_{\mathrm{HK}}$ in Figure 6, we see the same story in a slightly different way. First, there is a lack of a convection-dominated regime, which would be indicated by an increase in jitter for the least active stars in these plots. These stars are therefore activity-dominated, and because they have reached our instrumental floor, it is unclear whether they have lived long enough to have spun down to their jitter minimum. We further note by comparing to other mass bins that the activity dependence is diminished for lower masses- that is to say that even the most active stars in this group only have an $\mathrm{RV}$ jitter of $10-20 \mathrm{~m} \mathrm{~s}^{-1}$. Although activity and RV jitter are still strongly correlated, the dependence is much weaker, with a 

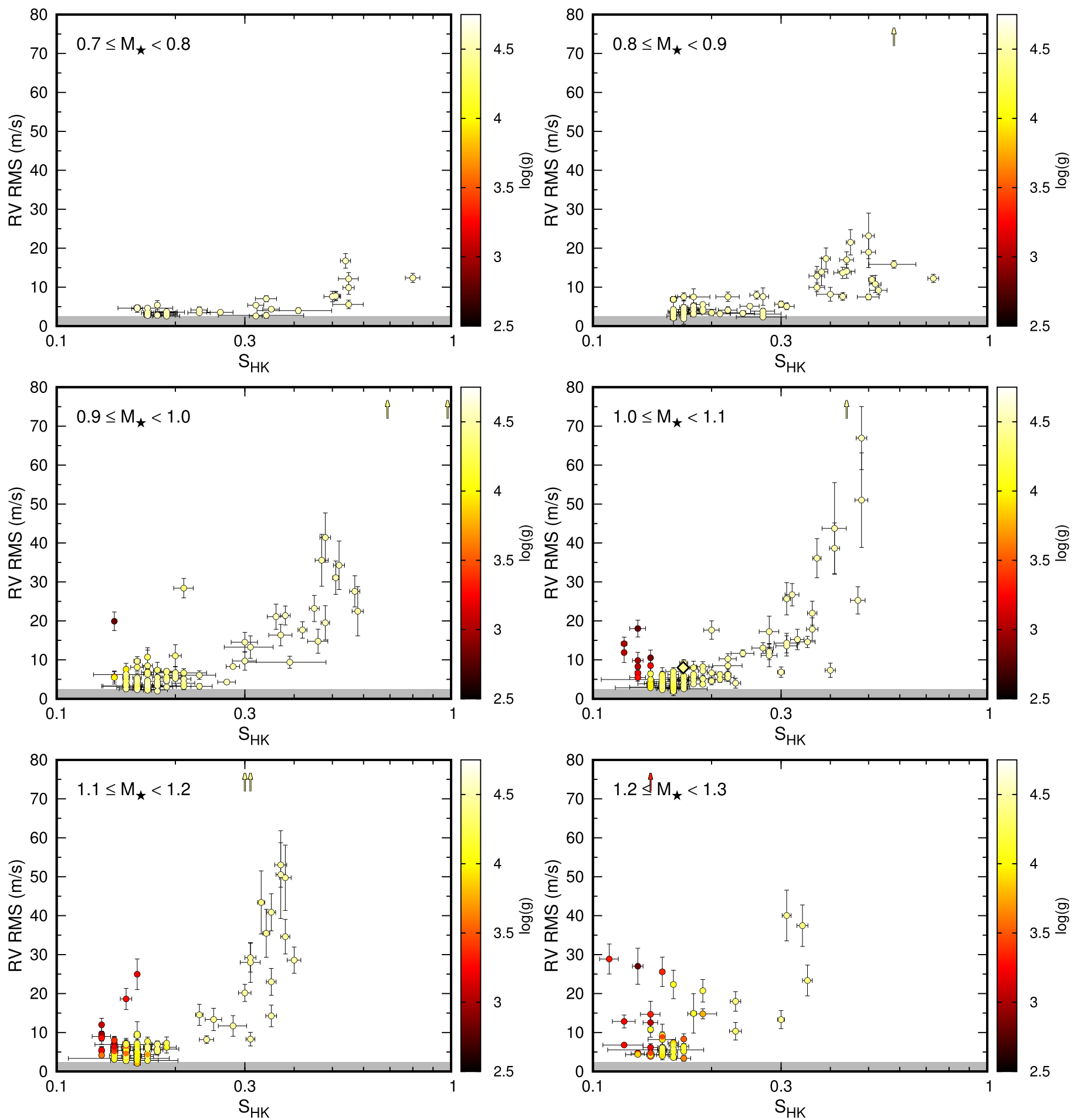

Figure 6. Same as the second panel of Figure 2 but separated by mass bin. The highest mass bins in our sample are seen in Figure 7. Solar values are plotted as a diamond in mass bin $1.0 \leqslant M_{\star}<1.1 M_{\odot}$, as in Figure 4. The shaded region at the bottom of the plots shows the typical Keck-HIRES instrumental uncertainty.

slope six times shallower than that in the $1.0-1.1 M_{\odot}$ bin (slopes of 15 and 98). This echoes the results of Isaacson \& Fischer (2010), who reported this "sweet spot" for spectral type $\mathrm{K}$ stars, which exhibit relatively low levels of RV jitter across all measured activity levels.

We wish to note that for this group of stars, there appears to be a discrepancy between the theoretical ZAMS and the ZAMS one would infer from looking at the vertical pileup of the "active main sequence." That is, the observed surface gravities appear to be systematically lower than predicted by stellar models. We see this only in this sample of low-mass stars, and we attribute this to be due in part to the calibration of $\log g$ in B17, which used asteroseismic surface gravities to calibrate the measured spectroscopic surface gravities. As such, the asteroseismic sample was mostly evolved stars (and therefore massive enough to have evolved within the lifetime of the universe). Therefore, there are very few calibration points for lower mass dwarfs, and it is expected that the measured surface gravities would be less certain. Despite the larger uncertainties and tendency to underestimate the surface 

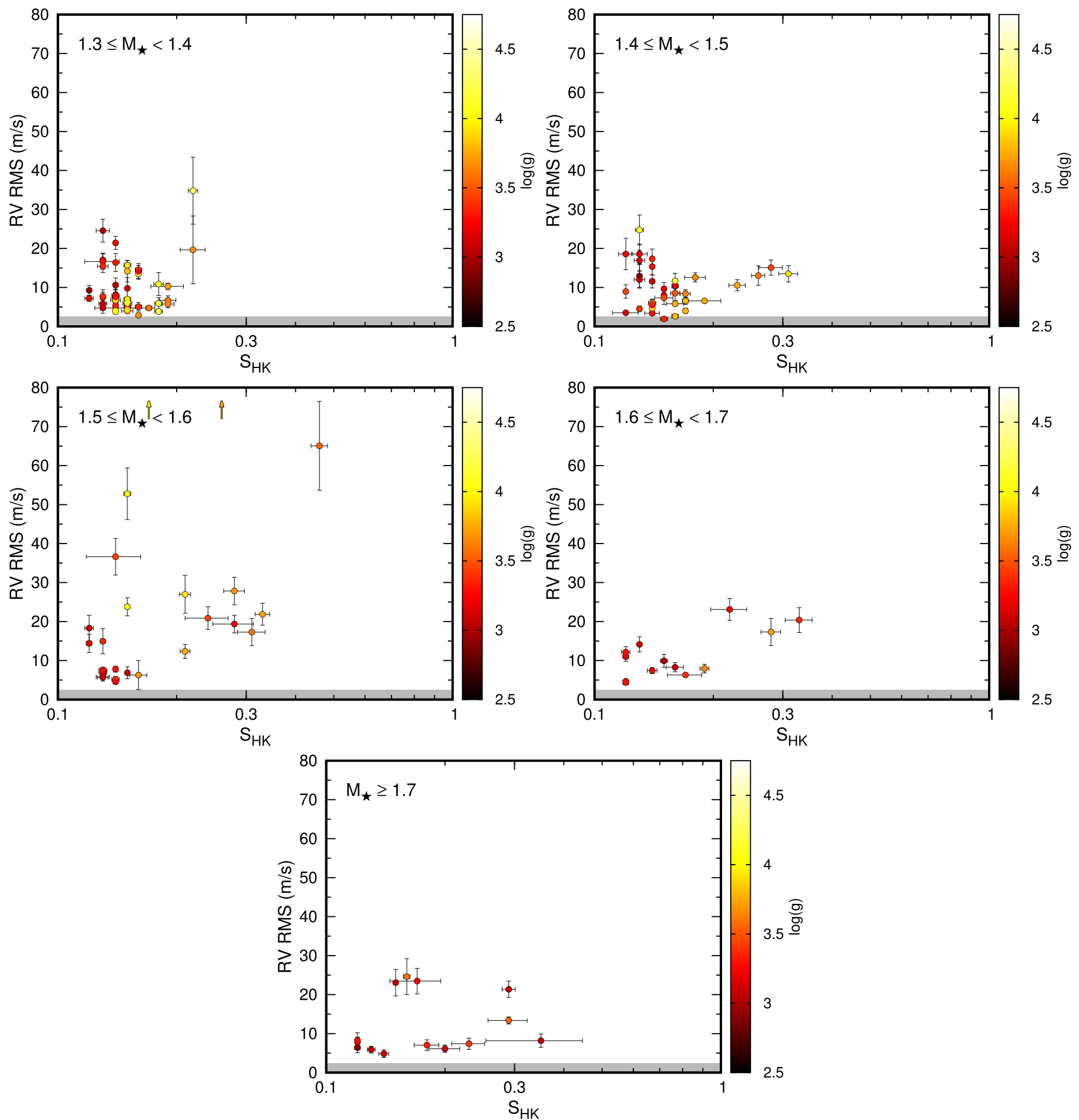

Figure 7. Same as Figure 6, for the larger mass bins.

gravities for the lowest mass dwarfs in our sample, our results hold. We note that this effect is reduced when using the isochrone-derived surface gravities given in B17, which are otherwise disfavored.

We are limited in our sample of low-mass stars by the lower temperature limit of B17. We expect similar trends to hold (i.e., low jitter regardless of activity) for stars below $0.7 M_{\odot}$ and therefore expect them to continue to be a "sweet spot" for planet searches. However, the inability to have fully spun down on the timescale of the age of the universe means that these stars may only be able to spin down to a level of $\mathrm{RV}$ jitter that could be above what we see in our lowest mass bins. Further, we expect different manifestations of activity for stars that are fully convective and so we avoid speculating about the RV jitter of such stars. New infrared spectrographs (Carmenes, HPF, SPIROU, iSHELL) will provide better studies for the behavior of RV jitter for these fainter $\mathrm{M}$ dwarfs.

We remind the reader that we see no evolved stars in this set of stars due to the main-sequence lifetimes being longer than 


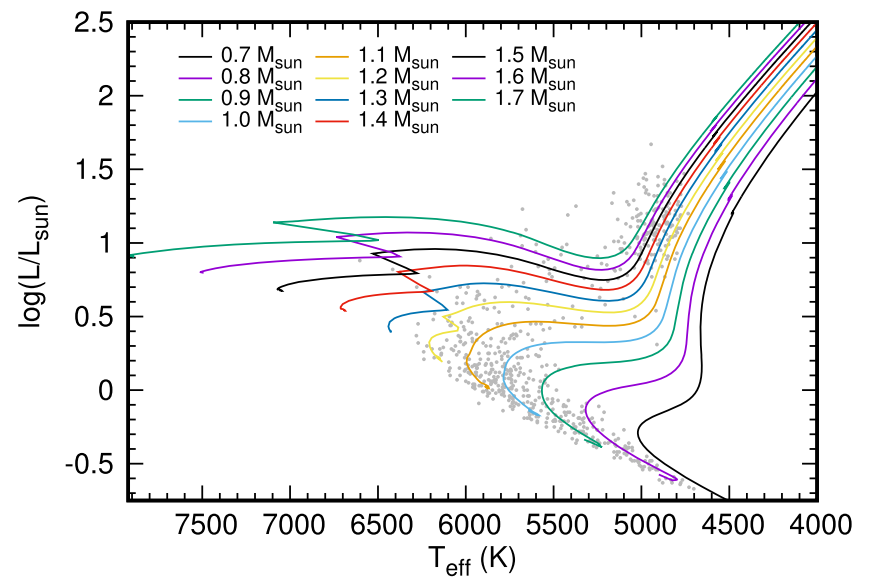

Figure 8. MESA evolutionary tracks used for the theoretical granulation and oscillation components of RV jitter (Equations (9) and (7)) as a function of surface gravity for each mass bin in Figure 5. The CPS sample in this work is shown by the light gray points for reference.

the age of the universe (as is indicated by the washed out lines in Figure 4 that show the theoretical convection component).

\subsubsection{Solar-mass Stars: $0.9 \mathrm{M}_{\odot} \leqslant \mathrm{M}_{\star}<1.5 \mathrm{M}_{\odot}$}

Although the highest mass in this range would typically not be considered "solar mass," we find that the RV jitter of stars in this range of masses behaves very similarly. Stars of roughly solar mass up to $1.5 M_{\odot}$ exhibit the following trends in $\log g$ (seen in Figures 4 and 5). These stars start as active stars that then move vertically down the "active main sequence" as they spin down. However, it is clear from these plots that stars in this mass range do not reach their jitter minimum before beginning to evolve to lower $\log g$. The "jitter minimum" instead occurs for stars that have measurably evolved, but before the stars get so big that RV variations caused by convection become dominant. ${ }^{14}$ This transition region from activity-dominated jitter to convectiondominated jitter comes mostly from the loss of magnetic activity. The color gradient seen in the stars in Figure 4 in the mass range $1.0-1.1 M_{\odot}$ near the jitter minimum at $\log \sim 4.3$ clearly shows this transition as stars become magnetically quiet. From there, a star follows the general path shown in the purple line, with RV jitter dominated by convection: first, we predict, by granulation and later by oscillations.

In terms of activity, Figures 6 and 7 show the same trends in a different manner. In these plots, it is easy to see the relation between activity and RV jitter. We clearly see when comparing the $0.9-1.0 M_{\odot}$ mass bin with the $1.0-1.1 M_{\odot}$ mass bin that the most active stars in the higher mass bin have higher levels of RV jitter. We expect this to hold generally: the most active stars of higher masses have higher RV jitter, continuing with what we saw in the lower mass bins in Section 4.1.1. This is confirmed by the slopes increasing with activity from the 0.9 to $1.0 M_{\odot}$ bin (slope of 62) to the 1.0 to $1.1 M_{\odot}$ bin (slope of 99), to the 1.1 to $1.2 M_{\odot}$ bin (slope of 132$) .{ }^{15}$ In other words, despite the fact that the range of $S_{\mathrm{HK}}$ values decreases with

\footnotetext{
${ }^{14}$ This is less clear in the $0.9-1.1 M_{\odot}$ mass bins because we are unable to fully probe the jitter minimum for these stars, given the instrumental uncertainty. However, it is still suggested in the plot that the jitter minimum occurs at lower $\log g$ values among the main-sequence stars in this bin.

${ }^{15}$ The increasing slope is also affected by the fact that $S_{\mathrm{HK}}$ is not normalized between spectral types (e.g., the most active stars in the 1.1-1.2 $M_{\odot}$ bin are all below 0.4 whereas the highest in the mass bin below it are below 0.5 ).
}

increasing mass, the range of RV jitter values is observed to increase substantially, with 97th percentiles in RV jitter of $12.38,18.99,31.09,36.09$, and $43.402 \mathrm{~m} \mathrm{~s}^{-1}$ for the first five bins of Figure 6 . However, our ability to probe this trend for intermediate-mass stars $\left(\sim 1.3-1.5 M_{\odot}\right)$ is hampered by selection effects, outlined below. The convection-dominated regime is not as clearly defined as it is in the plots of $\log g$. In general, we see that below a certain activity level (different for each mass bin), stars exhibit high levels of RV jitter again.

From the plots for these stars, it is clear that both activity and evolutionary state of the star are useful for selecting stars that are RV quiet as we see a well-defined transition between the activity-dominated regime and the convection-dominated regime.

We wish to briefly discuss the inclusion of intermediatemass stars in this grouping. Stars of intermediate mass $\left(1.3-1.5 M_{\odot}\right)$ on the main sequence are not suitable for RV observations. Their hot temperatures produce few absorption lines in their spectra, and their rapid rotation broadens any absorption features they may have to the point where precise RV measurements are not possible. Therefore, to study planets around intermediate-mass stars, surveys like the "Retired A-Star Survey" (Johnson et al. 2006) have examined their evolved stages where they have both cooled and spun down, allowing for precise RV measurements. Therefore, we do not see many stars in the "ZAMS" portion of these figures for stars above $1.3 M_{\odot}$. We also must point out that this mass is not surprisingly near the Kraft break (Kraft 1967). Above this mass range, dwarf stars rotate too rapidly for precise RV measurements. The Kraft break also pinpoints the region where stars begin to have very thin (or nonexistent) convective envelopes. If they lack a convective envelope, this further complicates our analysis because they would no longer be magnetically active, as convection is a required condition for our current understanding of magnetic dynamo (Parker 1955). Instead, spindown for these stars occurs after the main sequence as they gain a deepening convective envelope (Kippenhahn et al. 2012; van Saders \& Pinsonneault 2013). Any attempts at obtaining RVs of intermediate-mass stars on the main sequence would probably contain large amounts of RV jitter, likely dominated by pure uncertainty in measuring a precise velocity from the rotationally broadened absorption features ${ }^{16}$ rather than magnetic activity.

Instead, intermediate-mass stars show up in our sample once they have appreciably evolved and become amenable for precision RVs. Van Saders \& Pinsonneault (2013) argue that stars above the Kraft break are able to spin down rather quickly post-main sequence due to the rapid rotation during the onset of magnetic winds coupled with the fact that these stars are substantially expanding and increasing their moments of inertia (more so than in lower mass stars). As such, massive stars are able to spin down from their main-sequence rotations $\left(v \sin i>70 \mathrm{~km} \mathrm{~s}^{-1}\right)^{17}$ to velocities more amenable for $\mathrm{RV}$ measurements $\left(v \sin i<20 \mathrm{~km} \mathrm{~s}^{-1}\right)$ in a relatively short amount of time. Despite the rapid spin-down, their descent toward lower RV jitter is not as vertical as seen in the lower mass stars. This is presumably because the subgiant lifetime is shorter than the timescale to spin down and so these stars then

\footnotetext{
16 The A-F stars studied in Galland et al. (2005) were seen to exhibit RV uncertainty in the range of 50-300 $\mathrm{m} \mathrm{s}^{-1}$. Additional work has shown that RV uncertainty can be as much as $\sim \mathrm{km} \mathrm{s}^{-1}$ in O-type stars (Williams et al. 2013). 17 Based on Kraft (1967).
} 
stay active down to very low $\log g$. They therefore travel along a diagonal path downward and to the right in the log $g$ plots as they leave the main sequence.

When looking at the $\log g$ plots for the intermediate-mass stars (first two panels of Figure 5), there is evidence for an "active main sequence" where stars are spinning down and traveling vertically downward with the decrease in RV jitter. However, there are only a few points that indicate the presence of an active main sequence. When we look at the $S_{\mathrm{HK}}$ plots, there is very little evidence of a trend with activity for the active stars, casting doubt on the any evidence of an active main sequence for these stars. Given the low significance, the appearance of an "active main sequence" in the log $g$ plots can be explained away by measurement uncertainties $(\log g, M, \mathrm{RV}$ rms). However, these stars still clearly show the rising floor of the convection-dominated regime as they evolve through the subgiant and giant phases. Despite expecting stars in the range of $1.3-1.5 M_{\odot}$ to have different trends from the solar-mass stars, it is clear from the $\log g$ plots that these stars follow the trends seen among the solar-mass stars instead of the trends seen in the higher mass stars.

We are limited in this sample by several selection effects. Probing the full effect of activity is challenging because of the nature of exoplanet searches, which have predominantly searched around inactive stars, rightly expected to have lower levels of RV jitter. Added to this is the effect of rotational broadening. At higher masses, stars have thinner and thinner convective envelopes and are unable to fully spin down on the main sequence. We only see the initial effects of this in the stars in this mass range. The effects are strongest in the next mass range.

\subsubsection{High-mass Stars: $\mathrm{M}_{\star}>1.5 \mathrm{M}_{\odot}$}

Astrophysically, the stars in this set follow the same ideas of transitioning from being activity-dominated to being convection-dominated, but with two distinct differences as seen in the plots of $\log g$. First, as mentioned above, these stars are not able to spin down until they gain a convective envelope, which occurs in the subgiant phase for these stars. Second, their evolutionary timescales are considerably shorter, such that the spin-down timescale is of order their evolutionary timescale. This means they are seen to be moving diagonally downward to the right as they spin down, decrease in jitter, and expand into a giant star. Evidence of this trend was seen in the previous set of stars, but it becomes very clear when looking at the behavior of this set. The transition from activity-dominated to convectiondominated is therefore spread out in $\log g$, and in fact, the most evolved stars in our sample have only just hit the theoretical convection limit. This means that these stars are still spinning down and activity becomes the dominant predictor of RV jitter, rather than evolution.

We see slight evidence of this when looking at the plots of $S_{\mathrm{HK}}$ for this set of stars when compared with the first two bins of Figure 7. The active and rapidly rotating main-sequence stars in the first two bins $\left(1.3-1.4 M_{\odot}\right.$ and $\left.1.4-1.5 M_{\odot}\right)$ are avoided in target selection and as a result there appears to be a negative correlation between $S_{\mathrm{HK}}$ and RV rms because the stars are in the convective-dominated regime where RV jitter increases with time as they continue their final spin-down. For the stars in mass bins above $1.5 M_{\odot}$, the sign flips, and we see a stronger positive trend again, most likely because they evolve more quickly and were more rapidly rotating to start
Table 4

Estimated RV Jitter Minimum by Mass

\begin{tabular}{lcccc}
\hline \hline $\begin{array}{l}\text { Mass Bin } \\
\left(M_{\odot}\right)\end{array}$ & $\begin{array}{c}j_{\min } \\
\left(\mathrm{m} \mathrm{s}^{-1}\right)\end{array}$ & $\begin{array}{c}\text { Location of } j_{\text {min }} \\
\log g\end{array}$ & $\begin{array}{c}\text { ZAMS } \\
\log g\end{array}$ & $\begin{array}{c}\text { TAMS } \\
\log g\end{array}$ \\
\hline $0.8-0.9$ & $\leqslant 2.5$ & 4.5 & 4.629 & 4.294 \\
$0.9-1.0$ & $\leqslant 2.5$ & 4.4 & 4.587 & 4.251 \\
$1.0-1.1$ & $\leqslant 2.5$ & 4.35 & 4.535 & 4.210 \\
$1.1-1.2$ & $\leqslant 2.5$ & 4.25 & 4.500 & 4.104 \\
$1.2-1.3$ & 3.5 & 4.2 & 4.429 & 4.017 \\
$1.3-1.4$ & 4 & 4 & 4.339 & 3.959 \\
$1.4-1.5$ & 4 & 3.8 & 4.297 & 3.907 \\
$1.5-1.6$ & 5 & 3.3 & 4.284 & 3.844 \\
$1.6-1.7$ & 5 & 3.25 & 4.297 & 3.873 \\
$>1.7$ & 5.5 & 3.1 & 4.302 & 3.873 \\
\hline
\end{tabular}

with, so they are still. Unfortunately, our data are too sparse in these bins to fully support these claims. Instead, the data appear to be merely consistent with the framework we have adopted for the evolution of RV jitter as seen in the lower mass bins and extrapolated to these bins. At least in the $1.6-1.7 M_{\odot}$ bin, it seems that activity is a better predictor of RV jitter.

An important caveat to remember is that this sample does not contain many evolved giant stars. It is reasonable to expect that if we observed giant stars with $\log g<3$, we would see the rise of the convection-dominated floor and therefore see the negative trend with $S_{\mathrm{HK}}$ below about 0.2 . It is therefore only true that activity is the better predictor of RV jitter for this specific set of stars in our sample. Additionally, as mentioned above, main-sequence stars of these masses were avoided in RV observations and so we continue to lack stars close to the ZAMS, and in this set of the highest mass stars we barely have any stars before the TAMS.

The two regimes of RV jitter as they relate to low-mass (Section 4.1.1), solar-mass (Section 4.1.2), and high-mass (Section 4.1.3) stars can be briefly summarized as follows. Low-mass stars evolve very little during the transition from activity-dominated to convection-dominated RV jitter. It is clear that for these stars, the spin-down timescale is much less than their main-sequence lifetimes, as expected (van Saders \& Pinsonneault 2013). Higher mass stars evolve quite dramatically before they complete the transition from activitydominated to convection-dominated RV jitter. The two reasons for this are that (1) higher mass stars (above the Kraft break) are unable to spin down while on the main sequence and so all spin-down occurs in the subgiant and later evolutionary stages, and (2) higher mass stars evolve more quickly. The key result from this is that the jitter minimum occurs at later stages of evolution for higher mass stars.

\subsubsection{Location of the Jitter Minimum}

Given this picture for the stellar evolution of RV jitter as a function of mass, we provide a rough estimate of the value and location (in terms of $\log g$ ) of the jitter minimum for each mass bin in Table 4 . These estimates are performed by eye. For each mass bin, we simply follow the RV jitter evolution in the $\log g$ plots, starting first at the active main sequence and following that sequence downward as the stars spin down. For the lowest mass stars, they hit the instrumental floor in a vertical strip that makes it quite easy to estimate the location of the jitter minimum. As they appear to be at the instrumental floor, we can only say that their jitter is likely below $2.5 \mathrm{~m} \mathrm{~s}^{-1}$. For the 

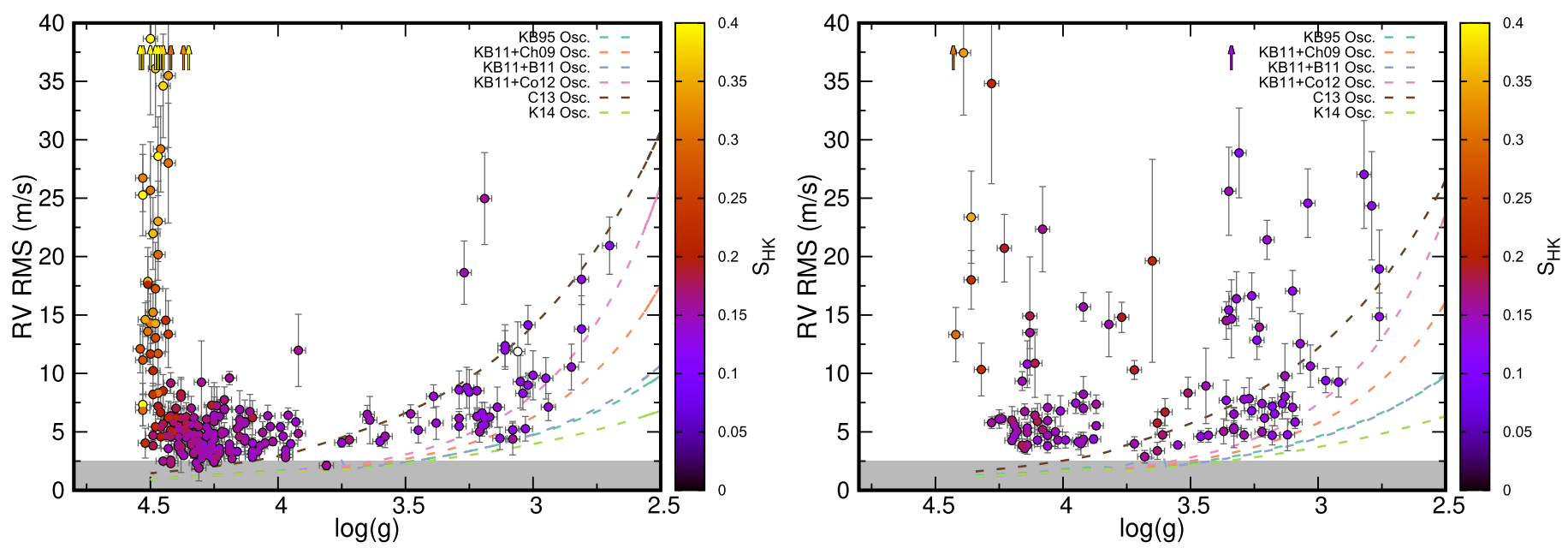

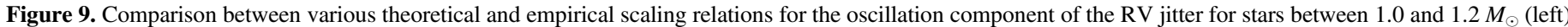

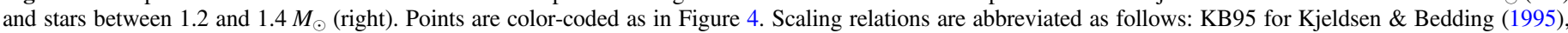

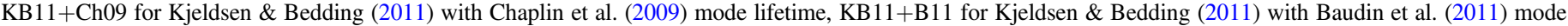

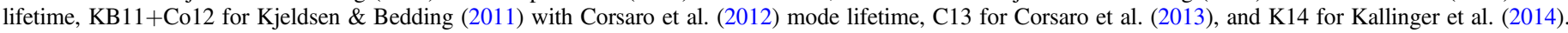

mass bins in the solar range, we follow the spin-down of the active main sequence in increasingly gradual transitions, as suggested by the floor of the data. When they reach the instrumental floor or appear to essentially flatten out, we again estimate the $\log g$ at which this first appears to happen (toward higher $\log g$ ). For the highest mass stars, we follow the diagonal path downward until it roughly reaches the modeled convective floor.

\subsection{Validation of the Oscillation Scaling Relation}

Now that we have discussed the major trends and observational limitations, we briefly comment on the validation of the scaling relation used for the oscillation component of the $\mathrm{RV}$ jitter. We tested several different scaling relations for the oscillation component of the RV jitter that can be found in the literature. A detailed account of each relation would detract from the purpose of this paper. We find that many of the relations agree with the general trends, and we cannot with confidence claim that one relation is preferred by the data. To compare all of the different scaling relations, we check using two wider mass bins, $1.0-1.2 M_{\odot}$ and $1.2-1.4 M_{\odot}$, by examining how well the resulting scaling relation followed the jitter floor. We find that the effects are seen most clearly toward the end of the subgiant regime and into the giant regime, which is why we use these bins where our sample is large and the floor is fairly well defined. All tested relations can be seen in Figure 9. We find that KB11+Co12 (Kjeldsen \& Bedding 2011 oscillation scaling relation with the Corsaro et al. 2012 mode lifetime scaling) shows the best agreement with the floor of our data by eye, and we use this as light empirical evidence in favor of these relations. As can be seen, the other relations either showed too sharp or too shallow of an increase with $\log g$. We note that KB11+Ch09 (Kjeldsen \& Bedding 2011 relation with the Chaplin et al. 2009 mode lifetime) also does a decent job fitting the RV jitter floor. We choose the Corsaro et al. (2012) mode lifetime over the Chaplin et al. (2009) lifetime for its ability to better fit the floor at the lowest surface gravities, seen best in the right panel of Figure 9.

To further test our scaling relation, we make use of observations of HD 142091, a fourth-magnitude subgiant for which asteroseismic RV observations were made specifically to target stellar p-mode oscillations. The observations were made over several hours on two separate nights in mid-2013. The first night (June 24) contains 246 observations over a $4 \mathrm{hr}$ span. The second night (June 30) is separated by 6 days and contains 151 observations over a $2 \mathrm{hr}$ span. Each night of observation shows a clear sinusoidal variation in the RVs due to the stellar p-mode oscillations, shown in Figure 10. The remaining observations of this star show evidence of a planet (Baines et al. 2013). To calculate the RV jitter for this star, we first subtract out the planet. The RV jitter of the residuals to this fit is then largely driven by the nearly 400 observations that show the stellar oscillations. Because the rms for the two nights of observations is significantly smaller than the scatter for the remaining observations, we remove these from the jitter calculation and obtain an RV rms of $7.6 \mathrm{~m} \mathrm{~s}^{-1}$ compared to the rms during the two nights of high-frequency observations of $2.6 \mathrm{~m} \mathrm{~s}^{-1}$, which provide a good check of our expected oscillation component. We show this with an "X" in the 1.5-1.6 $M_{\odot}$ mass bin of Figure 5 and note that this is the only star in our sample for which we have resolved stellar oscillations. The " $X$ " lands almost exactly where the scaling relation in Equation (7) (Kjeldsen \& Bedding 2011; Corsaro et al. 2012) predicts it to be. We note that the blue dashed line showing the oscillation component of the RV jitter shown in Figure 5 is for a nominal $1.5 M_{\odot}$ star. We can go one step further and compare the measured and expected oscillation amplitudes based on the actual stellar properties for this star.

Using the stellar properties of HD $142091\left(M=1.5 M_{\odot}\right.$, $\left.T_{\text {eff }}=4781 \mathrm{~K},[\mathrm{Fe} / \mathrm{H}]=0.25, \log g=3.26, R=4.85 R_{\odot}\right)$, the expected oscillation amplitude is $3.293 \mathrm{~m} \mathrm{~s}^{-1}\left(2.944 \mathrm{~m} \mathrm{~s}^{-1}\right.$ when using the Chaplin et al. 2009 mode lifetime). For both nights of observations, we find the best-fit sinusoid to determine the amplitude of stellar p-mode oscillations. The best-fit sinusoid for the June 24 observations has an amplitude of $2.778 \mathrm{~m} \mathrm{~s}^{-1}$. The amplitude for the June 30 observations is slightly smaller: $2.511 \mathrm{~m} \mathrm{~s}^{-1}$. Both observations show very good agreement with the predicted amplitudes from Kjeldsen \& Bedding (2011) and Corsaro et al. (2012), which we show in Figure 10. The excellent agreement between the measured and expected amplitudes for a massive evolved star is validation for 

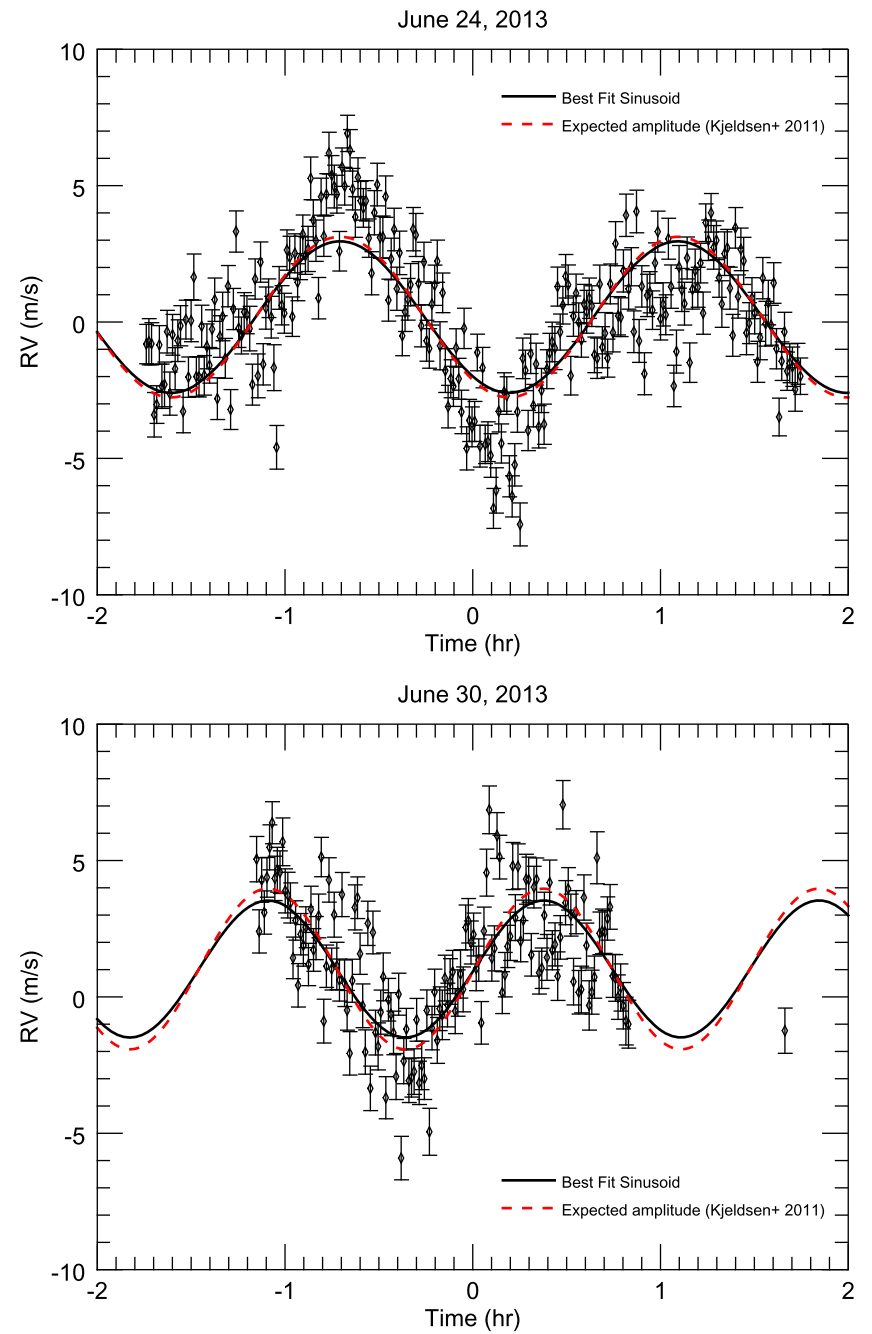

Figure 10. Observations of HD 142091 targeting stellar oscillations. The black curve shows the best-fit sinusoid for the observations on 2013 June 24 (top panel) and 2013 June 30 (bottom panel). The red curve in each panel shows the same sine curve but with the predicted oscillation amplitude based on the scaling relation in Equation (7) (Kjeldsen \& Bedding 2011; Corsaro et al. 2012).

the oscillation scaling relation used in this work, which has been scaled to the Sun.

\subsection{Other Features of Jitter Evolution}

Here we briefly highlight a few other results and features noticed in the jitter evolution plots (Figures 2, 4-7).

\subsubsection{Jitter-above-the-floor Stars}

Our primary concern has been to establish the "jitter floor" and explore trends among stellar types. After establishing the jitter floor, we examined stars that appeared to be well above the floor and here we note two stars that have significantly higher RV jitter than similar stars (at $\log g \sim 3.2$ ) and are well above our relation. These two stars, HD 207077 and HD 128095 , are in fact stars that showed lower jitter in the early stages of the vetting process when the blind fitting had subtracted Keplerian signals.

HD $207077\left(M=1.13 M_{\odot}, \log g=3.27, \sigma_{\mathrm{RV}}=18.62 \mathrm{~m} \mathrm{~s}^{-1}\right)$ has a strong planet candidate signal at around 600 days and is among those listed in Luhn et al. (2019) as planet candidates. We are nearly convinced by this planet; however, we have decided to not count it as a planet until more observations are made. However, the above-average jitter for this type of star provides strong evidence that this planet is real (see Luhn et al. 2019). Indeed, if we subtract our best planet model, we get an RV rms of $6.69 \mathrm{~m} \mathrm{~s}^{-1}$, where it would fall along our relation. This validates our conservative vetting procedures and supports our confidence that none of the features in our plots are the products of any subjectivity in our vetting.

HD $128095\left(M=1.2 M_{\odot}, \log g=3.35, \sigma_{\mathrm{RV}}=25.59 \mathrm{~m} \mathrm{~s}^{-1}\right)$ shows strong evidence of both a long-period planet and a shorter period planet. However, given the small number of observations, we expect that our two-planet fit might underestimate the true jitter, as the system will be overfit. For now, we have simply removed the long-period planet; however, it results in a large jitter for this star. Given that it is well above our relation, we believe this provides further evidence that the two-planet model is closer to correct.

We notice several other high-jitter stars in the various plots, but the above two cases show clear examples of using the jitter floor to identify stars that appear to be out of place, indicating the presence of additional RV variation that has not been accounted for, namely planets. This type of identification is useful only for large offsets typical of Jovian-type planets but is still useful for prioritizing targets and optimizing the use of our resources.

\subsubsection{Active Subgiants}

We observe from Figure 2 a sample of stars with $3<\log g<3.5$ that are well above our jitter floor. These stars also show high levels of magnetic activity, which is generally unexpected for subgiant stars. However, this is the result of using a broad stellar sample covering a wide range of masses and evolutionary stages. As explained above, these are merely intermediate-mass stars that are still spinning down and evolving quickly before they can lose their magnetic activity. This is evidenced by the lack of any stars in this range of surface gravities that appear to have high jitter and abnormally high activity when looking at the plots separated by mass bins. We also note that a decent fraction of these active subgiants also have stellar companions, which could play a role in the observed increased activity, which was also noted in Isaacson \& Fischer (2010).

\subsubsection{Upturn in Jitter for Giant Stars}

We also notice when examining Figure 2 an upturn in RV jitter around $\log g \sim 2.75$. We are still unable to confirm that this is indeed a real feature of RV jitter evolution and not merely a few high points. If it is indeed real, this could perhaps indicate that the oscillation scaling relation has a sharper scaling with $\log g$ than the theoretical relations (Kjeldsen \& Bedding 2011; Corsaro et al. 2012) suggest. Given that it is only apparent in Figure 2 for our entire sample and less so in the same plots broken into mass bins, it is likely not real. However, our sample includes very few stars that have evolved to $\log g \sim 2.75$, and so the lack of evidence in the plots broken up by mass could merely be due to small sample size for these types of giant stars. A future goal is to extend this further and increase our sample of giant stars for precisely this reason. 


\subsubsection{Theoretical Bump in RV Jitter for Intermediate-mass Stars}

As can be seen in Figures 4 and 5, for stars above roughly 1.2 $M_{\odot}$, the theoretical oscillation and granulation components to RV jitter go through a period of increased RV jitter from about $\log g=4-3.75$ for the $1.2 M_{\odot}$ stars and moving to later evolutionary stages $(\log g=3.75-3.5)$ for the most massive stars in our sample. We find that this region corresponds to the onset of hydrogen-shell burning, which has the effect of restructuring the star. This occurs differently in stars above $\sim 1.2 M_{\odot}$ than for lower mass stars and can be seen in the evolutionary tracks for these stars as the period where the stars move back up and left on the H-R diagram as they temporarily become hotter and more luminous (see Figure 8). As a relatively small effect, it is likely only noticeable in purely theoretical tracks and is unlikely to be observable empirically due to slightly different stellar parameters ( $T_{\mathrm{eff}}, M, S_{\mathrm{HK}}$, $[\mathrm{Fe} / \mathrm{H}])$ between stars in the sample. Even with the higher precision offered by next-generation RV instruments, we expect that this bump might only be observed with a dedicated sample of nearly identical stars with similar observing strategies that specifically target granulation effects, and even then we do not expect it to be very likely.

\section{Discussion}

The conclusions drawn from this investigation have relied heavily on the examination of the RV jitter floor. We remind readers that our vetting process for calculating RV jitter has undoubtedly included many stars that have additional nonstellar components, which are inflating the measured RV jitter. However, by examining the floor of the RV jitter, we expect that we have primarily focused on those stars for which all dominant nonstellar components of the RV jitter have been removed.

Our results here have very serious implications for our understanding of RV jitter. We have shown the relationship between a star's evolutionary state and its RV jitter. In fact, RV jitter seems to track stellar evolution quite well, tracing mainsequence (and post-main-sequence) spin-down and the subsequent decrease in magnetic activity until a star becomes inactive, and its RV jitter follows the increase in convective power as both the characteristic size of a granular region and the p-mode oscillation amplitude grow with decreasing surface gravity. While the basic path is the same for every star, stars of different masses have subtle differences in where these transitions occur relative to the main-sequence lifetimes.

From this work, it is clear that RV jitter depends primarily on three stellar parameters: stellar mass, surface gravity, and magnetic activity. With these three parameters, we can establish an expected RV jitter. Future work will analyze this in more depth, as well as various methods to predict jitter. We also plan to investigate which types of stars have systematically large or small discrepancies between the observed and predicted jitter.

Furthermore, the basis of this work is to better understand the astrophysical drivers of RV jitter in order to better identify suitable RV targets. The following section highlights the important implications of this work for RV surveys.

\subsection{Implications for RV Surveys}

We break our implications into three broad categories: implications for informing target selection for RV surveys, prioritizing targets for RV follow-up, and identifying stars with abnormally high jitter.

\subsubsection{Target Selection for RV Surveys}

The RV community has long been aware of the relation between RV jitter and magnetic activity. Our results as they relate to magnetic activity align with previous results, with the main insight that binning by mass shows very clear relations rather than purely spectral type. We observe cleaner relations between jitter and activity due primarily to improved stellar parameters and thorough approach to calculating RV jitter. RV surveys will continue to avoid active stars, especially those that are higher mass, as they are seen to exhibit higher jitter than low-mass stars (as high as $60 \mathrm{~m} \mathrm{~s}^{-1}$ in $1.1-1.2 M_{\odot}$ compared to less than $20 \mathrm{~m} \mathrm{~s}^{-1}$ in $0.8-0.9 M_{\odot}$ ). In other words, in terms of $\mathrm{RV}$ jitter, more-massive stars are more sensitive to activity. ${ }^{18}$

In our analysis of $\log g$, we identified the jitter minimum for stars of mass $0.7<M_{\star}<1.7 M_{\odot}$ (see Table 4). This will enable RV surveys to tailor their stars to those expected to be at jitter minimum, and could expand exoplanet searches into regimes that have not been previously explored, although we note that an efficient target selection requires knowledge of the stellar mass, evolutionary status ( $\log g$ ), and magnetic activity (e.g., $S_{\mathrm{HK}}$ ). Our understanding of planetary system formation and evolution is hampered by our inability to detect planets, particularly in RVs in a wide variety of regimes. With this study, we can begin to strategically address this.

More generally, this study enables a more refined selection at a time when more precise and accurate stellar parameters are more readily available. It also enables surveys to better select specific focus points, for example subgiants, main-sequence stars at jitter minimum, more-evolved stars with sufficiently low astrophysical jitter, etc. It also highlights areas for focused observations for further jitter studies, which may enable a refined understanding of jitter in areas where we currently suffer from selection effects, thus permitting planet searches in sparsely populated areas of the jitter-mass-log g-activity space.

\subsubsection{Target Prioritization for RV Follow-up}

Similarly, we expect that this framework will be invaluable for prioritizing targets for RV follow-up. While we advise prioritizing stars with low expected jitter and avoiding stars with high expected amounts of RV jitter, we acknowledge that this will not stop RV teams from giving high priority to targets that present the most scientifically interesting cases, regardless of the expected jitter. However, in these cases, we encourage using the estimated or expected amplitude of RV jitter as well as knowledge of the expected dominant source of RV jitter to better prepare and select proper observing strategies, in an

\footnotetext{
18 We note that it is unclear why exactly this is the case. Under a purely spotmodel assumption, the larger radii of more-massive stars would naively lead one to assume that RV jitter would decrease with activity, due to the relatively smaller spot size compared to the stellar disk. Observing the opposite effect therefore might indicate that the spots themselves are larger on more-massive stars, or perhaps have a higher spot coverage. But there are many sources of activity-induced jitter, not solely spots. Indeed, some stars have high RV jitter dominated not by spots but by bright magnetic regions. We refrain from speculating on this point as it is still unclear exactly how activity manifests in stars of different masses and in turn how that translates to the measured RV. Lastly, it is worth reminding readers that the calcium H\&K lines are formed in the chromosphere of stars and the velocities are measuring photospheric effects.
} 
attempt to model or remove the RV jitter. For example, Dumusque et al. (2011) and Medina et al. (2018) give observing strategies for mitigating the effects of granulation as well as p-mode oscillations. Further, several studies have shown promising results in modeling stellar RV variability due to magnetic suppression of convective blueshift and show that proper longitudinal coverage and simultaneous photometry can significantly reduce variations (Aigrain et al. 2012; Haywood et al. 2014).

\subsubsection{Identifying Easy Planet Candidates}

As we have already seen in this work, we can use these relations to identify stars that have unusually high jitter compared to the expected value (Section 4.3). In these cases, the high RV jitter is likely due to unsubtracted companions. By comparing the observed jitter to the expected jitter, one can easily identify potential planet candidates and pick out stars that would benefit from additional observations. For example, stars in the mass range $0.9<M_{\star}<1.1 M_{\odot}$ at $\log g \sim 4$ that have an RV rms of more than $10 \mathrm{~m} \mathrm{~s}^{-1}$ are good candidates for potential planets.

\subsection{Limitations}

\subsubsection{Observational Biases in the CPS Sample}

In using data from the CPS, it is important to highlight some of the limitations imposed due to the nature of the survey. As mentioned previously, we see an instrumental noise floor of about $2.5 \mathrm{~m} \mathrm{~s}^{-1}$, and so we are unable to say anything about the RV stability of stars below that level. For some stars in our sample, we have measured RV rms less than $2.5 \mathrm{~m} \mathrm{~s}^{-1}$. We have opted to report these as measured to ensure consistency, because the instrumental uncertainty indeed varies from star to star, and we expect many stars to be RV stable below the $2.5 \mathrm{~m} \mathrm{~s}^{-1}$ level; however, great care should be taken with these stars.

Furthermore, the CPS did not follow any specific observing strategy for their targets. This means that the cadence and duration of observations can vary dramatically from star to star, and even for a single star throughout its observation history. Because the astrophysical processes that drive stellar RV jitter as described in this work all operate on different timescales (minutes to years), the cadence of observations for a given star will likely probe some of these processes more than others, which will affect the measured RV rms. As was shown with HD 142091, the high-cadence sampling to trace out the p-mode oscillations resulted in the majority of the observations being taken over a time span shorter than the typical timescale for other sources of RV variation, and as a result, those sources were not reflected in the overall rms. In general, the stars in this sample are observed infrequently enough that no single process should dominate the RV jitter, justifying our choice to add the theoretical granulation and oscillation components in quadrature as they will both be evenly and randomly sampled.

We also suffer from observational biases in the CPS survey, which have largely targeted inactive main-sequence stars. As a result, our leverage on activity and evolution effects on RV jitter is not as strong as it could be. In both of these areasmore active and more evolved-stars in general have fewer observations. In terms of activity, this likely arises from observing a given star for a period of time, before noticing that it had high jitter and thereby removing it from the list of targets for further observations. We also find that many of the most active stars were added more recently to the sample in an effort to explore young stellar systems as part of the Formation and Evolution of Planetary Systems Spitzer Legacy Program (Meyer et al. 2006) and so have not had as long of a baseline to gather many observations. In terms of evolution, we previously noted how the stars in the "retired" A star survey have been added more recently and also suffer from shorter time baselines and fewer observations. Adding to this is the tendency of these stars to be slightly more massive than solar and host planets on longer periods, both of which work to reduce the expected semiamplitude of potential planets. Thus, these stars require more observations than usual to disentangle planet signals and add to the likelihood of planetary signals remaining in our data despite our best efforts to remove them all.

\subsubsection{Choice of Number of Observations Threshold}

In this work, we have chosen to measure the jitter only for those stars with 10 or more RV observations. This limit, while arbitrary, was based on having sufficient observations to see signs of a planet signal, linear trend, or other nonstellar phenomenon in the RV time series. In practice, we find that the threshold for accurately calculating jitter to the $2.5 \mathrm{~m} \mathrm{~s}^{-1}$ level requires closer to 20 or 30 observations. However, we have a large number of stars in our sample that have between 10 and 20 observations , and so we have opted to make 10 our threshold to include these stars, given that our goal is to identify the jitter floor. In particular, most of the stars with 10-20 observations lie in the areas where we get most of our leverage (active stars and evolved stars), which is why it was important to include them. By making the threshold 10 observations, we are able to build up a statistically significant sample to define the jitter floor for these areas where we suffer from observational bias at the expense of adding several stars with jitter above the floor.

\section{Summary and Conclusions}

In this work, we have presented a comprehensive analysis of RV jitter that builds upon previous analyses in several ways. A major improvement is the additional years of observations from which we calculate jitter. Further, our results hinge upon the precise stellar parameters provided by B17, most notable of which are the accurate measurements of $\log g$. To calculate jitter, we focus on removing any RV variations that are not due to surface features on the star itself. This primarily means removing planetary and stellar companions, but also includes removing velocities during transit for some stars that show Rossiter-McLaughlin effects, an in-depth search for outliers, and other unusual circumstances that may lead to nonastrophysical RV variations. We impose a conservative subjective vetting technique that we apply on a star by star basis. By imposing a general method of attempting a one-planet fit to each star, we can tailor the technique to each star individually, iterating with new constraints on the fit until we favor or reject a companion fit. By not imposing a one-size-fitsall model to subtract out planets and other effects from our heterogeneous sample, we are able to verify the measurements of RV jitter for each star in our sample.

We first examined the evolutionary dependence of RV jitter by sorting our sample into mass bins of width $0.1 M_{\odot}$. By 


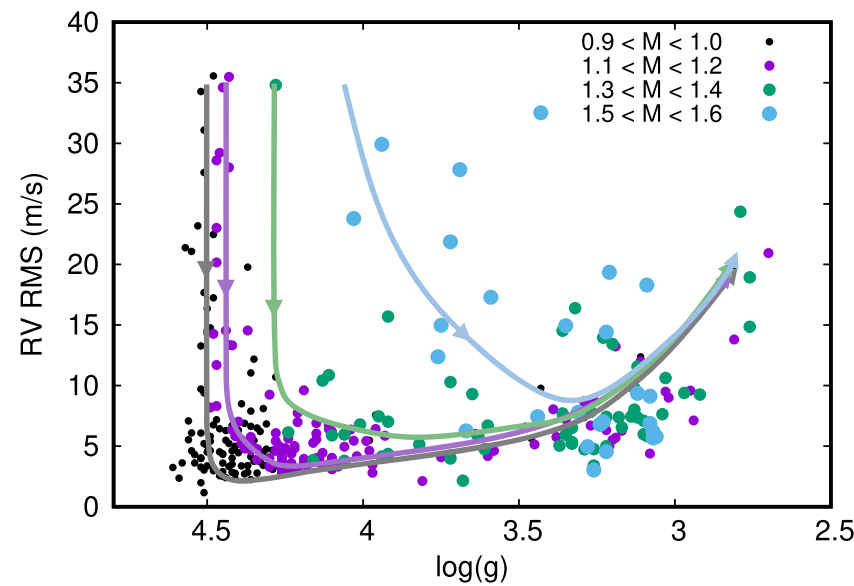

Figure 11. Schematic of jitter evolution using data for stars in four mass bins. The bins are discontinuous to better highlight the effect of mass on jitter evolution. Lines have been drawn (not fit) to represent the general evolutionary path suggested by the data for each mass bin. Here it is clear how more-massive stars begin to evolve before fully spinning down, such that the most massive stars move diagonally down and to the right as they transition from activitydominated to granulation-dominated and eventually oscillation-dominated jitter.

doing so, we observe empirical evidence of two regimes of RV jitter: activity-dominated and convection-dominated. Drawing from these observations, we conclude that RV jitter tracks stellar evolution and that most stars pass through the following stages of RV jitter:

1. ZAMS stars are born with high magnetic activity that drives large RV jitter.

2. As a star spins down on the main sequence, it loses angular momentum through magnetic winds, and RV jitter decreases with decreasing magnetic activity.

3. As a star becomes magnetically inactive, or "quiet," the dominant driver of RV jitter is surface convection. The transition from magnetically driven to convectively driven RV jitter defines the minimum level of RV jitter in a given star's evolution.

4. As a star moves through the subgiant and later giant regimes, the $\mathrm{RV}$ jitter increases as the p-mode oscillation amplitude increases and the number of granules on the star decreases due to the increased size of a characteristic granular region (which is related to the pressure scale height).

By investigating the different mass bins, we see subtle differences between different spectral types. As you go toward higher masses, the RV jitter minimum (and the transition from magnetically driven to convectively driven jitter) occurs at later evolutionary stages. This is due to both the shorter evolution timescales for higher mass stars and the lack of convective envelopes while on the main sequence for the highest masses in our sample (above $\sim 1.3 M_{\odot}$ ), which are thereby unable to spin down due to angular momentum loss from stellar winds until they evolve off the main sequence and gain a convective envelope. These trends with mass are shown in Figure 11, which shows a schematic of the jitter evolution for stars of various masses.

We also performed a similar analysis of RV jitter by examining the RV jitter as a function of magnetic activity. Our results are consistent with many previous studies of RV jitter which indicate strong correlation between RV jitter and magnetic activity among main-sequence stars. We confirm the result in Isaacson \& Fischer (2010) that for K dwarfs, RV jitter has a very weak dependence on activity. Instead, these stars show surprisingly low levels of RV jitter for even the most magnetically active stars.

Due to the nature of stellar evolution for more-massive stars, stellar spin-down for stars above the Kraft break does not occur until they have evolved off the main sequence and have gained a convective envelope. For these more-massive stars (above $\left.1.3 M_{\odot}\right)$, they remain in the activity-dominated regime throughout the subgiant phase. For these stars, activity becomes the better predictor for evolved star RV jitter rather than convection.

In summary, we observe three different classes of stars in terms of RV jitter:

1. Low-mass stars $\left(<0.9 M_{\odot}\right)$ that are strictly activitydominated (i.e., have not evolved enough to reach their convective phase)

(a) Despite the wide range of $S$ values, all of these stars seem to exhibit relatively low jitter.

(b) Not only have these stars not evolved enough to become convectively driven, but there is evidence for a lower mass limit where stars have not yet spun down enough to reach jitter minimum.

2. Solar-ish mass stars $\left(0.9 \leqslant M_{\star}<1.5 M_{\odot}\right)$ that display activity and convection-dominated phases

(a) All of these stars, including F dwarfs, display activityand convection-dominated regimes. The two regimes meet in a "jitter minimum," meaning that even F dwarfs, typically avoided by RV planet searches, can be amenable to RVs (see Luhn et al. 2020).

(b) The jitter minimum for these stars occurs on the main sequence or late in its main-sequence lifetime, near or right at the TAMS.

3. More-massive stars $\left(>1.5 M_{\odot}\right.$, beyond the Kraft break) which only enter the RV surveys when they are evolved and for which activity once again becomes the key predictor of RV jitter because these stars have now developed convective envelopes (and presumably solar dynamo-like activity).

(a) The most evolved of these stars in our sample appear to just be reaching the expected jitter minimum and show RV stability to $3-10 \mathrm{~m} \mathrm{~s}^{-1}$.

(b) Jitter minimum for these stars occurs well into the subgiant and giant phases.

Finally, we comment on the utility of these relations and follow-up work. From our sample of stars with well-measured stellar properties and RV jitter, we can build a model that is able to predict the expected RV jitter of a star given a variety of input stellar parameters and uncertainties. We expect that these results will be particularly beneficial for continued RV followup of planet candidates from K2 and TESS. We are also able to use these relations to identify stars that have noticeably higherthan-typical RV jitter, which indicates the potential presence of unsubtracted orbital companions. We can then select these targets for further observations to fully extract the companion.

In other words, our relations have highlighted two classes of stars for which RV observations are particularly useful: those stars that are at the "jitter minimum" of their evolution, and those stars that are already observed to have RV jitter well above the "jitter floor." Both of these classes present good 
regimes in which to look for planets. In addition to identifying promising targets for RV follow-up, our results can help inform target selection for RV surveys in a similar manner by identifying which stars are the most RV-stable.

The authors thank Fabienne Bastien for her founding role in initiating the investigation and defining the project. Her insight, discussions, and advice have greatly contributed to this work.

We thank John Brewer for many useful discussions and clarifications regarding spectroscopic stellar properties. We thank John Johnson for the use of data on HD 142091. We thank Raphaëlle Haywood and Tim Milbourne for their discussion on solar granulation and the HARPS data. We thank Sharon Wang for helpful discussions regarding solar oscillations.

Finally, we thank the referee, whose suggestions have helped clarify and focus the work presented here.

The data presented herein were obtained at the W. M. Keck Observatory, which is operated as a scientific partnership among the California Institute of Technology, the University of California, and the National Aeronautics and Space Administration. The Observatory was made possible by the generous financial support of the W. M. Keck Foundation.

The authors wish to recognize and acknowledge the very significant cultural role and reverence that the summit of Maunakea has always had within the indigenous Hawaiian community. We are most fortunate to have the opportunity to conduct observations from this mountain.

Keck time for this project has been awarded from many sources, primarily institutional time from the University of California, Caltech, NASA, and Yale. We thank the many observers and CPS team members who have worked over the decades to produce this invaluable data set.

This research has made use of the SIMBAD database, operated at CDS, Strasbourg, France; the Exoplanet Orbit Database and the Exoplanet Data Explorer at exoplanets.org.; and of NASA's Astrophysics Data System Bibliographic Services. This work was partially supported by funding from the Center for Exoplanets and Habitable Worlds, which is supported by the Pennsylvania State University, the Eberly College of Science, and the Pennsylvania Space Grant Consortium. This material is based upon work supported by the National Science Foundation Graduate Research Fellowship Program under grant No. DGE1255832.

\section{Appendix A \\ Calculation of Error on Jitter Measurements}

Because the RV rms is already a measurement of error and represents the standard deviation of the RV observations of a given star, we seek to calculate the uncertainty in our estimate of the true standard deviation (essentially the standard deviation of the standard deviation). For simplicity of notation, we find it helpful to define the variance

$$
v \equiv j^{2},
$$

which will help in transforming between the standard deviation $(j)$ and the variance. Where the standard deviation of the data is related to the second moment of the data set (the mean being the first moment), the variance of the sample variance is related to the fourth moment of the data. The exact statistical relation comes from Mood et al. (1974),

$$
\sigma_{j^{2}}^{2}=\sigma_{v}^{2}=\frac{\mu_{4}}{n}-\frac{j^{4}(n-3)}{n(n-1)},
$$

where $\mu_{4}$ is the fourth moment of the data, defined as

$$
\mu_{4}=\sum\left(\epsilon_{i}-\bar{\epsilon}\right)^{4}
$$

However, we are interested in knowing $\sigma_{j}^{2}$, not $\sigma_{j^{2}}^{2}$, and so we must use error propagation:

$$
\sigma_{j}^{2}=\sigma_{v}^{2}\left|\frac{\partial j}{\partial v}\right|^{2},
$$

which gives

$$
\sigma_{j}=\frac{\sigma_{j^{2}}}{2 j}
$$

The derivation of this formula assumes a normal distribution and does not account for individual error measurements. However our individual measurement uncertainties are largely homoscedastic, and our conclusions are not sensitive to the precision of our jitter uncertainties. Note also that this estimation of error in our calculation of RV jitter neither takes into account the $\chi^{2}$ of any subtracted fits nor the possibility of the data containing additional companions. It is merely a measurement of the uncertainty in calculating $\sigma_{\mathrm{RV}}$ from the residuals, $\epsilon$.

\section{Appendix B Notes on Individual Systems}

Here we list many individual systems and provide notes on what alterations we have applied to the RV time series when calculating RV jitter. We provide this for completeness and reproducibility. We additionally note stars that show strong correlations between the RVs and activity metric $S_{\mathrm{HK}}$, the vast majority of which have been independently extracted from the same spectra and published by Butler et al. (2017).

$$
\text { B.1. } 0.7 \leqslant \mathrm{M}_{\star}<0.8 \mathrm{M}_{\odot}
$$

HD 4628-HD 4628 shows evidence of an activity cycle. However, we notice several low outliers in the activity time series that suggest rapid decreases in activity. A closer investigation reveals that these "ramp downs" in $S_{\mathrm{HK}}$ occur at the beginnings and ends of the observing season. As a bright $(V=5.74)$ target, we interpret the sudden decreases in activity as observations taken during twilight and contaminated by the solar spectrum. We do not see evidence for any correlation between the activity and the velocities, and so we are satisfied with the reported jitter. We see similar effects in HD 26965, HD 69830, and HD 192310 below.

HD 10700_HD 10700 (also known as Tau Ceti) has four reported planets all with semiamplitudes of $0.55 \mathrm{~m} \mathrm{~s}^{-1}$ or less (Feng et al. 2017). As an RV standard star, it has a long baseline of observations (917 over $17 \mathrm{yr}$ ), and there are several obvious outliers. We remove eight points that have velocities more than $3 \sigma$ from the mean, which reduces the RV rms from 3.23 to $2.75 \mathrm{~m} \mathrm{~s}^{-1}$. After subtracting the best-fit multiplanet Keplerian, the RV jitter is essentially unchanged, 2.75-2.73, indicating that we are at the instrumental uncertainty for this star. 
HD 26965-HD 26965 has a proposed planet candidate with small semiamplitude by Díaz et al. (2018), who also acknowledge that it could simply be stellar activity masquerading as a planet. We choose to subtract the planet given the good agreement, but we note that the RV jitter is relatively unchanged (3.6-3.2 $\mathrm{m} \mathrm{s}^{-1}$ ). We also note an apparent activity cycle in this star, with low $S_{\mathrm{HK}}$ outliers reminiscent of the "ramp downs" seen in HD 4628. As another bright target $(V=4.43)$, we again attribute these outliers to solar contamination from chasing the star into twilight. We note that the lowest $S_{\mathrm{HK}}$ values for this star are unphysical $(<\sim 0.1)$, which could indicate errors in the $S_{\mathrm{HK}}$ extraction for this star.

HD 31560-HD 31560 shows a strong correlation between the activity and the RVs (Pearson coefficient 0.76). However, this correlation is based on only a few observations.

HD 97658 - HD 97658 shows a strong activity cycle that is not very correlated with the RVs (Pearson coefficient 0.31 after removing the known planet).

HD 100623-HD 100623 shows evidence of a long-term linear trend, which we have subtracted out.

HD 116443-HD 116443 shows evidence of an activity cycle, with no correlated RVs.

HD 170657-HD 170657 has three obvious outliers that have internal errors of about $25 \mathrm{~m} \mathrm{~s}^{-1}$. After removing these points, there was also a long-term linear trend evident in the data, which we have subtracted.

HD 220339-HD 220339 shows evidence of a possible activity cycle. More observations are necessary to confirm this signal. The activity time series show little to no correlation with the velocities.

HD 196124-HD 196124 shows a correlation between the activity and the RVs (Pearson coefficient 0.62). It is unclear whether the star is exhibiting cycling behavior as it undergoes a large decrease in activity over several years and then was not observed until several years later when it was higher in activity. It is likely indicative of a cycle, but more data is needed.

$$
\text { B.2. } 0.8 \leqslant \mathrm{M}_{\star}<0.9 \mathrm{M}_{\odot}
$$

HD 10476-HD 10476 shows evidence of a possible activity cycle that is weakly correlated with the RVs (Pearson coefficient 0.466).

HD 18143-HD 18143 has a long-term linear trend, which we fit with a Keplerian (RV jitter reduced from $4.7 \mathrm{~m} \mathrm{~s}^{-1}$ with a linear trend to 4.3 with a Keplerian fit). This star shows evidence of an activity cycle with period near 4500 days; however, the observations span at most one full period and likely only a fraction of a period so the exact cycle period is uncertain.

HD 20165-HD 20165 shows evidence of an activity cycle and correlated RVs (Pearson coefficient 0.66).

HD 42250 - The velocities of HD 42250 taken prior to the Keck-HIRES upgrade appear to be systematically offset from those taken post-upgrade. We include an offset of $6.6 \mathrm{~m} \mathrm{~s}^{-1}$, which reduces the RV jitter from 5.4 to $3.7 \mathrm{~m} \mathrm{~s}^{-1}$.

HD 69830 - HD 69830 has three published planets (Lovis et al. 2006), which we are able to recover. However, similar to HD 4628 above, we notice several low outliers in the activity time, suggesting rapid "ramp downs" in $S_{\mathrm{HK}}$ that occur at the beginnings and ends of the observing season, which are more apparent in this star than in HD 4628. As a bright $(V=5.95)$ target, we interpret the sudden decreases in activity as observations taken during twilight and contaminated by the solar spectrum. The low $S_{\mathrm{HK}}$ values do not affect the jitter and so a deeper dive into the cause of these low outliers is beyond the scope of this work. Nevertheless, this star remains an interesting target for investigating activity and the effects of solar contamination on measurements of stellar activity.

HD 114783-HD 114783 has a single planet with a 493 day period (Wittenmyer et al. 2009). The residuals to this fit show strong periodicity near 4300 days. However, this star has a noticeable activity cycle with a period near 3000 days. Given the lack of correlation and the fact that the planetary signal and activity signal are out of phase and not on the same period, we are inclined to believe the second planet.

HD 124106 - HD 124106 has an obvious outlier by eye that lies about $70 \mathrm{~m} \mathrm{~s}^{-1}$ above the rest of the observations. The RV jitter is reduced from 15.4 to $8.2 \mathrm{~m} \mathrm{~s}^{-1}$ when this outlier is removed. We note that this is a reasonably active star, and so it is possible that this outlier was due to a flare.

HD 125455-HD 125455 shows evidence of a possible activity cycle that is not correlated with the RVs.

HD 154345 - HD 154345 is a particularly interesting star. Wright et al. (2008) claimed the presence of a Jupiter twin in a $9 \mathrm{yr}$ orbit, while noting that the star has an activity cycle that is nearly the same period and in phase with the velocities. By looking at similar cycling $\mathrm{G}$ stars and not finding a correlation between the activity cycle and the RVs, the period and phase similarity of the proposed planet signal and the activity cycle were suggested to be coincidences. Later, with additional data, Wright (2015) reversed the claim of a planet after including several more years of additional observations that continued to show strong phase and period similarities. For this work, we again reverse the stance and decide to subtract off the planet because of the coherent RV signal. No other system in this sample with activity cycles that are correlated with the RVs shows such a clear and coherent Keplerian-like signal. Further, we note that within our own solar system, the solar cycle is very similar in period to Jupiter's period. With Jupiter being the dominant RV signal in the Sun, a distant observer could very well have the same conundrum for our own system. Either way, this remains a very interesting system to study more closely. For now, we have subtracted off the planet, resulting in an RV jitter of $3.2 \mathrm{~m} \mathrm{~s}^{-1}$, but note that the unaltered RVs yield an RV jitter of $12.7 \mathrm{~m} \mathrm{~s}^{-1}$. Given that it is not an overly active star, we find that $3.2 \mathrm{~m} \mathrm{~s}^{-1}$ matches a little more closely with similar stars (see Figure 6).

HD 165401-HD 165401 shows evidence of a long-term linear trend, which we have subtracted out.

HD 189733 - The velocities for HD 189733 contain a strong Rossiter-McLaughlin signal, which contributes to the residuals and the RV jitter. Removing the points during transit results in a change in RV jitter from 15 to $11 \mathrm{~m} \mathrm{~s}^{-1}$.

HD 192310 - HD 192310 is similar to HD 4628 and HD 69830 above where we observe "ramp downs" in the activity time series near the end of the observing season. As another bright $(V=5.73)$ target, we believe that this is another case of chasing a star into twilight, resulting in solar contamination or errors in the $S_{\mathrm{HK}}$ extraction. We note that the lowest $S_{\mathrm{HK}}$ values for this star are unphysical $(\lesssim 0.1)$. This star also has a longterm activity cycle with a period close to $10 \mathrm{yr}$.

HD 219538-HD 219538 appears to exhibit a possible decaying activity cycle, similar to HD 4915, which was identified as a possible Maunder minimum candidate in Shah et al. (2018) and is detailed in the next mass bin below. Unlike 
HD 4915, there is no correlation with the velocities for HD 219538.

HD 131156-HD 131156 has a long-term linear trend, which we have subtracted off.

$$
\text { B.3. } 0.9 \leqslant \mathrm{M}_{\star}<1.0 \mathrm{M}_{\odot}
$$

HD 4614-HD 4614 shows evidence of a long-term linear trend, which we have subtracted out.

HD 4915-HD 4915 shows a strong correlation between the RVs and the decaying activity cycle (Pearson coefficient 0.83 ). This star was recently identified as a possible Maunder minimum candidate, as the decaying activity cycle shows features similar to the the solar entrance into Maunder minimum (Shah et al. 2018).

HD 19467-The directly imaged T dwarf HD $19467 \mathrm{~B}$ has a period of more than $300 \mathrm{yr}$ and a mass of $52 M_{\text {Jup }}$ (Jensen-Clem et al. 2016). The period of this companion is too long for our observations and exceeds the $100 \mathrm{yr}$ period limit imposed by RVLIN. Therefore, we merely subtract the best-fit linear trend from the data, as we are unable to fit this companion.

$H D$ 20619-Despite not having a strongly noticeable activity cycle, there is a strong correlation between the velocities and activity measurements for HD 20619 (Pearson coefficient 0.73 ).

HD 24496-We have subtracted a linear trend from the velocities of HD 24496 and note the presence of an activity cycle.

HD 37213-We note that the RVs for this star prior to the 2004 upgrade are all clustered around -5 to -10 , with the postupgrade velocities centered more closely around 0 . However, given the small number of post-upgrade velocities, we simply take the full RV rms of $5.479 \mathrm{~m} \mathrm{~s}^{-1}$ and note that the RV rms of the pre-upgrade-only velocities is only slightly lower: $4.588 \mathrm{~m} \mathrm{~s}^{-1}$.

HD 40397-HD 40397 shows evidence of a long-term trend. However, removing a simple linear trend resulted in coherent periodicity remaining and so we have subtracted a full Keplerian signal. The RV rms after removing a line was $3.424 \mathrm{~m} \mathrm{~s}^{-1}$, compared to the $\mathrm{RV}$ rms after removing a Keplerian of $2.557 \mathrm{~m} \mathrm{~s}^{-1}$.

HD 42618-HD 42618 has a strong periodic signal near 4000 days, which appears by eye to be correlated with the activity cycle of this star, despite the medium correlation coefficient of 0.442 after subtracting out the planet (Fulton et al. 2016).

HD 46375-HD 46375 has an outlier, which we have removed, obvious from its $20 \mathrm{~m} \mathrm{~s}^{-1}$ uncertainty for that observation.

HD 90711-HD 90711 had an obvious outlier offset by about $200 \mathrm{~m} \mathrm{~s}^{-1}$. This observation also has errors that are an order of magnitude higher than the rest of the observations and so we remove this observation. The resulting time series has evidence of a long-term linear trend, which we subtract.

HD 96700-HD 96700 has been previously reported as having two planets (Mayor et al. 2011). The Keck velocities show no indication of such planets, and because the velocities from the initial discovery are not available, we are forced to disregard these fits. The Keck velocities also show a very strong correlation with activity (Pearson coefficient 0.91), driven in large part by one observation that has both low velocity and $S_{\mathrm{HK}}$. When we fix the orbital solution to the Mayor et al. (2011) orbital parameters, there is only a small decrease in RV jitter from 7.4 to $6.5 \mathrm{~m} \mathrm{~s}^{-1}$.
HD 99491-HD 99491 shows evidence of a strong activity cycle, which are correlated with the RVs (Pearson coefficient 0.74).

HD 105631-HD 105631 shows a strong correlation between the RVs and the activity (Pearson coefficient 0.72 ).

HD 109358-HD 109358 shows evidence of an offset between the pre- and post-upgrade velocities of about $8 \mathrm{~m} \mathrm{~s}^{-1}$. We include this offset, which reduces the jitter from 4.1 to $3.3 \mathrm{~m} \mathrm{~s}^{-1}$.

HD 136352-HD 136352 was previously reported as hosting three small planets (Mayor et al. 2011). However, the velocities used in the discovery were not reported, and the Keck time series is poorly sampled, leaving poor constraints on the fit. Given the number of observations for this star, a threeplanet fit to the Keck-HIRES data will be overfit and results in an $\mathrm{RV}$ jitter of $1.2 \mathrm{~m} \mathrm{~s}^{-1}$. In order to avoid overfitting, we opt to use the raw RV rms of $4.1 \mathrm{~m} \mathrm{~s}^{-1}$. We note that Udry et al. (2019) report an rms of $1.3 \mathrm{~m} \mathrm{~s}^{-1}$ for this star after fitting out all three planets.

HD 149806 - HD 149806 show evidence of an activity cycle with period near 3000 days $(8 \mathrm{yr})$.

HD 164595-HD 164595 has been previously reported to host a planet (Courcol et al. 2015). However, the Keck velocities by themselves do not show evidence for this planet and so we do not remove it.

HD 181234-HD 181234 shows a long-term linear trend, which we have subtracted.

HD 201219-HD 201219 shows a correlation between the activity and the RVs, with Pearson coefficient 0.84 .

HD 206374-HD 206374 shows a correlation between the activity and the RVs, with Pearson coefficient 0.82 .

HD 212291-HD 212291 shows a correlation between the activity and the RVs, with Pearson coefficient 0.60 .

HD 218868 - HD 218818 shows a strong activity cycle with period near 2000 days. This activity cycle is strongly correlated with the RVs (Pearson coefficient 0.759).

HD 8389-HD 8389 shows a slight correlation between the activity and the RVs, with Pearson coefficient 0.54 . The activity time series also shows evidence of an activity cycle.

HD 45652-HD 45652 has been previously reported to host a planet (Santos et al. 2008). However, the Keck velocities are discrepant with the SOPHIE, CORALIE, and ELODIE data for this star. We remain confused by this star and have not been able to track down why the Keck data do not agree at all with the ELODIE, CORALIE, or SOPHIE data, which agree with each other over many epochs. For now, we have simply not removed any planet from the Keck velocities. In fact, fixing the orbital parameters to those in Santos et al. (2008) increases the $\mathrm{RV}$ rms of the Keck velocities from 28.2 to $33.9 \mathrm{~m} \mathrm{~s}^{-1}$.

HD 62613-HD 62613 has slight evidence of an activity cycle.

HD 76445-HD 76445 shows a long-term linear trend, which we have subtracted.

HD 162232-HD 162232 shows a long-term linear trend, which has been subtracted. After subtracting, there is evidence for an additional companion (reducing the RV jitter from 12.2 to $8.2 \mathrm{~m} \mathrm{~s}^{-1}$ ), but not strong enough to subtract out. We only subtract the linear trend.

$$
\text { B.4. } 1.0 \leqslant \mathrm{M}_{\star}<1.1 \mathrm{M}_{\odot}
$$

HD 1461-There is an obvious outlier present in the time series for HD 1461. The median velocity error for all observations of 
this star is less than $1 \mathrm{~m} \mathrm{~s}^{-1}$, but the obvious outlier has an error of $70 \mathrm{~m} \mathrm{~s}^{-1}$. For this reason, we remove it from the set of observations for this star. We also note that the residuals to the known planets show strong periodicity near 4226 days. However, we find that this is the same period as the star's apparent activity cycle and appears to be in phase, so we do not attempt a third planet.

HD 45350 - HD 45350 is host to a known planet with a published period of 963 days (Endl et al. 2006). When using the best-fit parameters from Endl et al. (2006), we obtain a rather poor fit. In fact, our resulting best fit is worse than when using a simple blind fit for this star (reduced $\chi^{2}$ improves from 63 to 10 , and the jitter decreases from 8 to $4 \mathrm{~m} \mathrm{~s}^{-1}$ ). Given the large number of observations after the publication of the most recent best fit, we are inclined to believe that we have much better constraints on the orbital parameters now, which we report in Table 2.

HD 73256-HD 73256 was previously reported to host a short-period planet by Udry et al. (2003), who see a period of 2.5 days. This is the tallest peak in the periodogram of the Keck data; however, our best-fit with this period results in a scatter of $63 \mathrm{~m} \mathrm{~s}^{-1}$ compared to the $20 \mathrm{~m} \mathrm{~s}^{-1}$ seen in Udry et al. (2003). Because of the poor fit, we decide to not subtract this planet in the interest of observing the true jitter floor.

HD 92788-HD 92788 has two confirmed planets. ${ }^{19} \mathrm{We}$ find good agreement with HD 92788 b on a 325 day orbit. However, we are unable to find a believable two-planet fit with a second planet at 162 days, as claimed in Wittenmyer et al. (2013). Instead, we find strong evidence for a long-period second planet near 11,000 days. While this orbit does not have complete phase coverage, we believe it to be properly subtracting out a real companion. As we obtain more observations of this system, the long-period planet's orbital parameters will be further constrained. We do not observe any signs of an activity cycle for this star, and certainly not on the timescale of the more than $25 \mathrm{yr}$ period. For this reason, we are confident in our subtracting out this best-fit model. In addition, we have removed a single outlier which has a velocity error of $15 \mathrm{~m} \mathrm{~s}^{-1}$; the median error for the remainder of the observations is $1.3 \mathrm{~m} \mathrm{~s}^{-1}$.

HD 195019-For this system, we have split the pre-2004 data and the post-2004 data and treated them as two separate telescopes in the fitting procedure. We chose to do this after noticing that our original best fit caused nearly every point before the Keck upgrades to have a negative velocity in the residuals and nearly every point after the upgrade to have positive residuals. We take this as evidence that the fitting procedure is minimizing $\chi^{2}$ by simply straddling the two sets of data. The structure seen in the residuals also resembles the signal of a long-period planet. However, neither the pre-2004 nor post-2004 data show evidence of long-period trends and so we take it to simply be an incorrect offset between the pre-2004 data and post-2004 data. By including a $16 \mathrm{~m} \mathrm{~s}^{-1}$ offset (found by a best fit in RVLIN), we observe residuals that no longer appear to have a suspicious structure centered around the Keck upgrade date.

HD 217107-For this system, we simply take note of three points that appear to be outliers when examining the residuals. The best two-planet fit after using the orbital parameters from Wright et al. (2009) as initial guesses has three points that are

\footnotetext{
19 According to exoplanet.eu.
}

all significantly above the typical scatter of the residuals. However, the three points fall on consecutive observations and could represent real variations on the stellar surface during those observations. We do not find evidence in the reported errors on these velocities to warrant their removal and so we have kept them in the fitting and jitter calculations. Given the large number of observations on this star (142) and the relatively low $\left(4 \mathrm{~m} \mathrm{~s}^{-1}\right)$ jitter, we do not expect these three points to significantly affect the final jitter.

HD 150706-This star has a previously published planet (Boisse et al. 2012) using SOPHIE follow-up to an ELODIE planet candidate. Including the Keck data for this star, we find that it loosely agrees with the planetary parameters found by Boisse et al. (2012). However, the Keck data alone show strong correlation with $S_{\mathrm{HK}}(0.729$ Pearson correlation coefficient), indicating that the RV jitter observed could be entirely activity driven and not due to a planet. Indeed, the SOPHIE and ELODIE data have quite large errors compared to Keck. In addition, the long period of this planet means we do not have full phase coverage. In fact, the only points at RV maximum are the earliest ELODIE observations, which are the observations with the largest error bars. Thus, we are suspicious as to the veracity of this planet. Because of the low number of Keck observations for this star, we include the planet fit for now.

HD 207832-HD 207832 has two previously published planets (Haghighipour et al. 2012). With additional observations, we are unable to recover convincing fits. When examining the RVs, we notice a correlation with $S_{\mathrm{HK}}$, with a Pearson correlation coefficient of 0.6. Given that Haghighipour et al. (2012) do not discuss the activity of this star, we are inclined to believe that the jitter is activity driven. Further proof of this is from the periodogram itself. With the addition of several new observations since the initial discovery, we find that neither of the two periods found for the planets (162 days and 1307 days) correspond to the peak with the highest power in the periodogram, which is near 300 days. Despite our conservative approach in general, in this case we are conservative in the opposite way: we do not quite have enough evidence to definitively claim that these planets do not exist given the care that Haghighipour et al. (2012) put into demonstrating the planet's reality and so we use the best two-planet model.

HD 1388-This star shows signs of an obvious stellar companion, which we have fit out using RVLIN. Although our orbital parameters are highly uncertain and likely incorrect, it follows the by-eye curvature quite well and so we have kept it. The residuals show evidence of potential other companions; however, this would certainly result in overfitting. For now, we have excluded pre-2004 observations from our jitter calculation but not from our fit. We find that the uncertainties in the velocity measurements for this star before the Keck upgrade $\left(4-5 \mathrm{~m} \mathrm{~s}^{-1}\right)$ are substantially higher than after the upgrade $\left(\sim 1 \mathrm{~m} \mathrm{~s}^{-1}\right)$.

HD 8038-For this star, we find a very high correlation between $S_{\mathrm{HK}}$ and the RV observations (Pearson correlation coefficient 0.93 ). We therefore take the observed $\mathrm{RV}$ variations as pure jitter.

HD 9986-This star also shows a fairly strong activity correlation (Pearson correlation 0.5), but more importantly shows evidence of a strong activity cycle, with a periodogram peak near 1000 days, which is near the strongest peak in the $\mathrm{RV}$ periodogram. We expect that the observed cyclical RV variations are activity driven. 
HD 13931-For this star, we have removed an obvious outlier, whose velocity uncertainty of $8 \mathrm{~m} \mathrm{~s}^{-1}$ is significantly higher than the median uncertainty for this star $\left(1.2 \mathrm{~m} \mathrm{~s}^{-1}\right)$. This star also has a strong planet signal, which further shows that this point is an outlier.

HD 18803-HD 18803 shows evidence of a strong activity cycle, and has $S_{\mathrm{HK}}$ values that correlate well with the RV observations (Pearson coefficient of 0.51). We disregard any strong peaks in the RV periodogram of this star and take the $\mathrm{RV}$ variations for this star as jitter.

HD 32923-For this star, we notice a long-term trend in the RVs. We therefore subtract a linear fit for this star before fitting a blind fit to the data. The best-fit planet with a line removed does not meet our criteria and so we simply remove only the linear fit from the velocities.

HD 39881-HD 39881 has what appears to be either a very slight offset between the pre- and post-upgrade velocities or a very slight linear trend. The difference in RV jitter between these approaches is very small: $4.0 \mathrm{~m} \mathrm{~s}^{-1}$ when subtracting a linear trend and $3.8 \mathrm{~m} \mathrm{~s}^{-1}$ when applying an offset between the pre- and post-upgrade velocities (the raw RV rms is $4.5 \mathrm{~m} \mathrm{~s}^{-1}$ ). We choose to use the linear trend to better avoid overfitting.

$H D 52711-\mathrm{HD} 52711$ has an outlier in the $S_{\mathrm{HK}}$ time series. After removing this point, there is a slight correlation between the RVs and the activity (Pearson coefficient 0.61 ), which is why we disregard the blind-fit planet.

HD 68168 -HD 68168 has several observations in short succession that span nearly $40 \mathrm{~m} \mathrm{~s}^{-1}$. Other than these few points, the star appears to have some slight cyclical variation to it. However, we find that the $S_{\mathrm{HK}}$ values are well correlated (Pearson coefficient 0.61), indicating that these observed RV variations are simply activity-driven jitter. We have also removed two observations that were taken during a gibbous moon through 1-3 mag of clouds.

HD $233641-$ HD 233641 shows a very strong correlation between the activity metric $S_{\mathrm{HK}}$ and the measured velocities (Pearson correlation coefficient 0.95 ), again indicating activitydriven RV jitter.

HD 153458 - HD 153458 also shows a very strong activityRV correlation (Pearson coefficient 0.96), indicating that RV variations are driven by activity in this star.

HD 157347-HD 157347 has three outliers due to velocity errors more than 2.5 times the median errors for this star, although the velocity measurements themselves are consistent with the rest of the data. Nevertheless, we remove these three observations.

HD 157338 -HD 157338 has two obvious outliers in the RV time series. These are identified by eye and also by their large uncertainties $\left(6.3\right.$ and $\left.18 \mathrm{~m} \mathrm{~s}^{-1}\right)$ compared to the median uncertainty $\left(1.5 \mathrm{~m} \mathrm{~s}^{-1}\right)$ for this star. It has an obvious stellar companion with a long period that cannot be well constrained. We are able to find best-fit two-companion models with a planet and a stellar companion; however, we reject these fits based on the convenient lack of phase coverage near sharp RV peaks in models that find rather large eccentricities. We posit that more observations are necessary before we can definitively believe a two-companion model. We therefore restrict ourselves to circular orbits for the obvious stellar companion, reemphasizing that it is the jitter floor we seek. Our stated jitter of $11.5 \mathrm{~m} \mathrm{~s}^{-1}$ is likely an overestimate of jitter, but we are careful not to oversubtract (our best two-companion models yield a jitter of $\sim 4.7 \mathrm{~m} \mathrm{~s}^{-1}$ ).
HD 85689-HD 85689 has one extremely low point separated by more than $100 \mathrm{~m} \mathrm{~s}^{-1}$, but otherwise seemingly normal. We assume that this is an anomalous point, and we have removed it for that reason, which brings the jitter from 25 to $6 \mathrm{~m} \mathrm{~s}^{-1}$.

HD 92719- The by-eye inspection of this star shows what appears to be a strong activity correlation, despite a medium Pearson coefficient (0.46). Regardless, we find no evidence of Keplerian RV variations, and so we do not make any changes to this star.

HD 197076 - We see strong evidence of an activity cycle for this star, with a period of $\sim 2000$ days. This also corresponds to the peak in the RV time-series periodogram. The correlation between activity and velocity is quite strong (Pearson coefficient 0.63 ) and so we simply take everything as activity-induced jitter.

HD 12484-HD 12484 has one known planet (Hébrard et al. 2016) and is an active star with high jitter $\left(43 \mathrm{~m} \mathrm{~s}^{-1}\right)$. After subtracting out the planet, we find a strong activity correlation with the residual velocities (Pearson coefficient 0.74 ).

HD 45184-After removing an obvious outlier in both the activity and velocity time series, we find a correlation (Pearson coefficient 0.67 ) between the activity index and the residual RVs for HD 45184 after subtracting out the best-fit planet (Mayor et al. 2011).

$$
\text { B.5. } 1.1 \leqslant \mathrm{M}_{\star}<1.2 \mathrm{M}_{\odot}
$$

HD 13043 - The RV observations for HD 13043 show some coherence, which could be evidence of an activity cycle or a planet. Further observations are necessary to fully determine what is driving the apparent coherence and so for now, we do not remove any signals.

HD 107148 - HD 107148 has a purported planet with period of 48 days (Butler et al. 2006). We see no evidence for a planet at 48 days and instead find a strong peak near 77 days, which RVLIN finds as the best-fit period. When we remove our best fit for this 77 day planet, a tall peak in the periodogram shows up at $\sim 20$ days. Our best fit to a two-planet model is not convincing enough to accept. However, we note that this star is worth further observations to reliably reject or accept the presence of a second planet. For this work, we merely take the rms of the residuals to a one-planet fit as the RV jitter.

HD 141004-HD 141004 has an obvious outlier that we have removed. The error on this measurement was more than 2.5 times the typical error for this star, and the measured velocity is more than five times the mean jitter for this star. In this case, the resulting difference in jitter is not extreme: $5.126 \mathrm{~m} \mathrm{~s}^{-1}$ when the outlier is included and $5.007 \mathrm{~m} \mathrm{~s}^{-1}$ with it removed. However, the point remains a clear outlier and so we remove it.

HD 28005-HD 28005 also contains a very obvious outlier. The error is significantly more than 2.5 times the typical error for this star. Removing the outlier, our measurement of jitter reduces substantially from 13.14 to $7.24 \mathrm{~m} \mathrm{~s}^{-1}$.

HD 159222-HD 159222 shows a very strong correlation between RV observations and $S_{\mathrm{HK}}$, with a Pearson correlation coefficient of 0.69 . We therefore take the RV observations as activity-induced jitter.

$H D$ 182572-We have removed an outlier from the observations of HD 182572. The outlier is obvious by its larger than normal errors. However, the velocity of this observation is not largely displaced from the mean RV 
measurement and so the RV jitter is largely unaffected by the presence of this outlier.

HD 195564-HD 195564 shows evidence of a clear stellar companion, which we remove. The residuals to our best-fit stellar companion show coherence and what appears to be a somewhat sinusoidal signal. However, we are unable to arrive at a good two-body model and so we simply subtract the loosely fit stellar companion and treat the residuals as jitter.

HD 1293-HD 1293 has 12 Keck-HIRES observations spanning $5 \mathrm{yr}$ (mid-2007 to mid-2012). The RVs rise close to linearly over the course of these observations and so we subtract a linear trend for this star. There are strong deviations from this line, which could be evidence of an additional companion that cannot yet be subtracted out due to the limited number of points. This target presents a good case for future observations to correctly fit the long-term linear trend and to determine the nature of the additional perturbations to that line, whether it be pure RV jitter or an additional companion.

HD 77818 - HD 77818 shows evidence of a small stellar companion. There are enough observations to observe a slight curvature in the long-period trend; however, we resort to subtracting a linear trend until more observations are obtained.

HD 128095-HD 128095 also shows evidence of a longperiod stellar companion. For this star, the curvature is enough to produce a believable fit to the time series. However, the residuals to this fit show a variation of $\pm 30 \mathrm{~m} \mathrm{~s}^{-1}$. When we attempt a two-planet fit, we find a surprisingly good fit to the time series with a roughly circular 472 day planet and a fairly eccentric $(e=0.63)$ stellar companion. However, given that there are only 14 observations for this star, we expect that our two-planet fit has overfit, given that we are attempting to fit 14 data points with 12 free parameters. We therefore simply remove the long-period trend and encourage follow-up of this star to investigate the legitimacy of a second planet (in Section 4.3.1, we find further evidence that points toward the second companion being real).

HD 207077-The periodogram of the observations of HD 207077 exhibits a strong peak near 600 days. The resulting best-fit planet model presents a convincing case for a 606 day planet with eccentricity 0.20 . Despite the good fit and improved jitter from 18.66 to $6.69 \mathrm{~m} \mathrm{~s}^{-1}$, we decide to disfavor this planet for now and for our jitter analysis. In doing so, we can investigate how the raw jitter for this star matches that of a similar star and use this as a case to favor or disfavor the planet. HD 207077 is discussed further in Section 4.3.1.

HD 10212-The RV time series for HD 10212 shows a clear stellar companion that passes through periapse. However, observations are sparse and only contain two points with negative RVs near pericenter. This caused RVLIN a few problems in obtaining a good fit. We noticed that no model could perform a good fit for both of these points, with residuals for these points separated by more than $100 \mathrm{~m} \mathrm{~s}^{-1}$ (with every other residual close to or less than $100 \mathrm{~m} \mathrm{~s}^{-1}$ ). At first inspection, neither of these two points seemed to be a clear outlier. However, upon further investigation, we find that the median $\chi$ value for one of these observations was abnormally high, indicating that the RV was poorly measured due to a poor fit to the absorption in the spectrum itself. When we remove this point, our fit improves dramatically, from a jitter of $28 \mathrm{~m} \mathrm{~s}^{-1}$ (and reduced $\chi^{2}$ of 193) to $7 \mathrm{~m} \mathrm{~s}^{-1}$ (reduced $\chi^{2}$ of 23).
B.6. $1.2 \leqslant \mathrm{M}_{\star}<1.3 \mathrm{M}_{\odot}$

HD 8907 - HD 8907 is a fairly active star and shows good correlation between the activity and RV time series (Pearson coefficient 0.61). Given this and the lack of a best-fit model, we expect that all RV variations are driven by the activity of the star.

HD 28237-We notice a strong correlation between RVs and activity in this fairly active star (Pearson coefficient 0.58), suggesting that RV jitter in this star is activity driven.

HD 52265-We find little evidence of the 59 day second planet for this star published in Wittenmyer et al. (2013) and are unable to get a fit without invoking high eccentricity. Regardless, the best two-planet fit using Keck data results in an $\mathrm{RV}$ jitter of $4.1 \mathrm{~m} \mathrm{~s}^{-1}$, compared to $4.6 \mathrm{~m} \mathrm{~s}^{-1}$ in the one-planet fit. By selecting the one-planet fit, we have not changed the RV jitter enough to affect any results.

HD 74156-For this star, we find that the residuals to the two-planet fit are all below $0 \mathrm{~m} \mathrm{~s}^{-1}$ for data before the Keck upgrade (with a mean of $-8.7 \mathrm{~m} \mathrm{~s}^{-1}$ compared to a mean of $3 \mathrm{~m} \mathrm{~s}^{-1}$ post-upgrade), but not by more than the average scatter for the post-upgrade residuals. In fact, the lowest velocity residuals are all post-upgrade. For this reason, we have not split the pre- and post-upgrade velocities in the fit but have taken only the post-upgrade velocities when calculating the jitter.

HD 114613 - This is a star with a known planet (Wittenmyer et al. 2014) on a 3624 day period. However, the Keck data show an activity correlation (Pearson coefficient 0.695 ) and do not show evidence of this planet. We therefore consider the velocities to be purely activity-induced RV jitter.

HD 179949-After the discovery of a companion to HD 179949 in Butler et al. (2006), this star had additional observations starting again in 2009. After removing the bestfit companion, we notice that the pre-upgrade residuals cluster around $10 \mathrm{~m} \mathrm{~s}^{-1}$, while the post-upgrade observations cluster around $-10 \mathrm{~m} \mathrm{~s}^{-1}$. When including an offset in the fit, the RV jitter is reduced from 15 to $10 \mathrm{~m} \mathrm{~s}^{-1}$.

HD 188512-HD 188512 shows evidence of a long-term linear trend, which we have subtracted out.

HD 198802 - We find a strong activity correlation for HD 198802 (Pearson coefficient 0.78), which leads us to disregard the best-fit single-planet model and leave the RV variations as activity-driven jitter.

HD 11970 - After subtracting the stellar companion to HD 11970 , we note a correlation between the RVs and the activity (Pearson coefficient 0.73).

HD 25311-HD 25311 shows evidence of RV variation beyond simply activity-driven variations (given the lack of correlation between activity and velocities). We find that with a linear trend and a period of 70 days, we can reduce the RV jitter to nearly $10 \mathrm{~m} \mathrm{~s}^{-1}$. However, this fit relies on a convenient lack of phase coverage during the RV maximum of the orbit and so we are hesitant to accept this best-fit planet. We instead simply subtract a linear trend.

HD 25457-We remove an obvious outlier from this star. The outlier is not as obvious when simply comparing the uncertainties in the velocities because this star has rather large velocity uncertainties $\left(13 \mathrm{~m} \mathrm{~s}^{-1}\right.$ versus $\left.5 \mathrm{~m} \mathrm{~s}^{-1}\right)$. However, it is noticeable by eye as an outlier in that it is below $-175 \mathrm{~m} \mathrm{~s}^{-1}$ when nearly every other velocity for this star is within $\pm 30 \mathrm{~m} \mathrm{~s}^{-1}$.

HD 38801-After subtracting the best-fit companion to HD 38801 , we note a correlation between the RVs and the activity (Pearson coefficient 0.64). 
HD 88134 -HD 88134 shows evidence of a long-term linear trend, which we have subtracted out.

HD 117497-This star has the minimum number of observations for our jitter calculation (10) but over a very short baseline (close to 2 months). For this star, our jitter estimate is therefore relatively uncertain.

HD 144363-HD 144363 has clear evidence of a stellar companion (RV variations with semiamplitude $6 \mathrm{~km} \mathrm{~s}^{-1}$ ). However, the small number of points makes an exact fit difficult, and the residuals to the best fit remain of order $500 \mathrm{~m} \mathrm{~s}^{-1}$. This could suggest spectral contamination from the binary companion, leading to spurious RV measurements, but for now, it is unclear whether the poor fit is due to measurement error or the lack of complete coverage.

HD 154144-After subtracting a linear trend, we find a strong correlation between the RVs and the activity (Pearson coefficient 0.68 ).

HD 156342 - HD 156342 shows evidence of a long-term linear trend, which we have subtracted out.

$$
\text { B.7. } 1.3 \leqslant \mathrm{M}_{\star}<1.4 \mathrm{M}_{\odot}
$$

HD 21847-HD 21847 is another active star that shows strong correlation between activity and RVs (Pearson coefficient 0.78). The RV jitter for this star is relatively high $\left(34 \mathrm{~m} \mathrm{~s}^{-1}\right)$ but appears to be purely activity induced.

HD 39828-HD 39828 showed evidence of a long-period stellar companion due to a strong linear trend, decreasing by about $400 \mathrm{~m} \mathrm{~s}^{-1}$ over the eight and a half years of observations. Because there is no indication of curvature, we have subtracted a linear trend.

HD 95088-HD 95088 had an obvious outlier that we have removed. This outlier was identified by eye as it had a velocity measurement of $100 \mathrm{~m} \mathrm{~s}^{-1}$ that was removed from the remaining points. The $\mathrm{RV}$ jitter is decreased from 23 to $9 \mathrm{~m} \mathrm{~s}^{-1}$ once the outlier is removed.

HD 106270-HD 106270 shows a clear planetary signal (Johnson et al. 2011). However, it also shows a strong activity correlation (Pearson coefficient 0.645). Despite the strong correlation, the two signals do not seem to be entirely in phase, and the planetary signal has sufficient phase coverage to indicate that the planetary signal is indeed correct.

$$
\text { B.8. } 1.4 \leqslant \mathrm{M}_{\star}<1.5 \mathrm{M}_{\odot}
$$

HD 38529-HD 38529 shows evidence of a strong activity cycle; however, this cycle does not appear to induce correlated RVs. The planets found in Wright et al. (2009) are not in phase and show little signs of being activity-induced RV variations.

HD 63754-Despite obtaining a planet fit for HD 63754, we find that we are quite likely overfitting in this case. Instead, we simply subtract off a linear trend. Additional observations would confirm if the curvature suggested by the planet fit is indeed present.

HD 33142-HD 33142 has one previously known planet (Johnson et al. 2011). After subtracting out the best-fit planet, we find evidence for an additional planet near 860 days, seen as a planet candidate in Luhn et al. (2019). We compare the oneplanet, one-planet plus trend, two-planet, and two-planet plus trend fits, and find that there is not strong enough evidence to accept either of the two-planet fits. We therefore take the oneplanet fit with a trend as it resulted in a lower $\chi^{2}$ than the oneplanet fit alone. This system is worth further scrutiny to determine whether there is indeed a second planet in this system. If it does, our current jitter measurement will be slightly inflated: $8.7 \mathrm{~m} \mathrm{~s}^{-1}$ instead of $6.0 \mathrm{~m} \mathrm{~s}^{-1}$.

HD 99706-HD 99706 was originally published as a oneplanet system with a linear trend (Johnson et al. 2011). Later, Bryan et al. (2016) used additional observations to refine the fit and published a two-planet solution. With the addition of a few more observations, we find that the current data set is inconsistent with the Bryan et al. (2016) two-planet solution. We simply use a one-planet plus trend solution.

HD 140025-We note that the RV observations of HD 140025 have a strong correlation with activity (Pearson coefficient 0.785 ).

HD 142091-HD 142091 was discussed in detail in Section 4.2. The majority of the observations of this star were obtained on two separate nights in 2013 to capture stellar p-mode oscillations. These observations therefore skew the rms toward the lower values expected from purely stellar oscillations. We therefore remove these two nights and calculate the RV rms of the remaining observations. The oscillation observations are used separately to validate the theoretical scaling relations used in this work.

HD 155413-HD 155413 shows evidence of a stellar-mass companion, and so we have simply subtracted a linear trend.

HD 219553 - HD 219553 has a potential planet based on a one-planet fit. However, the periodogram does not show a clear, sharp peak at the best-fit period. Given the few observations and poor phase coverage of the best-fit planet fit, we opt to disregard the best-fit one-planet solution and treat it as pure RV jitter. If there is indeed a planet, the RV rms drops from 18.6 to 5.8. As such, this is a system where we may be able to use its height above the expected jitter floor to provide additional evidence for or against the one-planet solution.

HD 224032-This system shows evidence of a long-term linear trend, which we have subtracted off.

$$
\text { B.9. } 1.5 \leqslant \mathrm{M}_{\star}<1.6 \mathrm{M}_{\odot}
$$

HD 67767-The velocities of HD 67767 pre-upgrade show much larger variation due to several high points than the postupgrade velocities $\left(14 \mathrm{~m} \mathrm{~s}^{-1}\right.$ versus $\left.6 \mathrm{~m} \mathrm{~s}^{-1}\right)$. However, the post-upgrade velocities show evidence of an activity correlation. If the velocities are indeed correlated with activity, then the high-velocity measurements pre-upgrade could simply be activity induced. Therefore, we find it necessary to include all velocities in the calculation of $\mathrm{RV}$ jitter $\left(11 \mathrm{~m} \mathrm{~s}^{-1}\right.$ when combined).

HD 18645-HD 18645 shows a slight correlation between activity and RV (Pearson coefficient 0.538 ).

HD 22682-HD 22682 has unusually large errors in the measured velocities (median $6.6 \mathrm{~m} \mathrm{~s}^{-1}$ ). It is not a faint star, nor is it rapidly rotating. Rather, it is bright $(V=6.67)$ and slowly rotating $\left(v \sin i=0.8 \mathrm{~km} \mathrm{~s}^{-1}\right.$.).

HD 31423 - The velocities for HD 31423 show a rising trend in velocities spanning several hundred $\mathrm{m} \mathrm{s}^{-1}$ and crossing $0 \mathrm{~m} \mathrm{~s}^{-1}$ from mid-2011 to mid-2012. A single point removed from the rest by more than a year (late 2013) has a measured velocity near $3000 \mathrm{~m} \mathrm{~s}^{-1}$. As the latest observation, we are unable to constrain a fit with this point included. We do not expect this to be an errant point, but in order to accurately measure the jitter, we remove this point from the time series. We subtract the resulting rising trend as a simple linear trend.

HD 72440 - HD 72440 presents a very interesting case. We observe what appears to be a strong downward linear trend spanning $400 \mathrm{~m} \mathrm{~s}^{-1}$. However, when examining the $S_{\mathrm{HK}}$ time 
series for this star, we notice that they correlate very strongly with the RVs with a Pearson coefficient of 0.984. While the RV trend is almost certainly indicative of a stellar companion, the corresponding very large drop in $S$ is hard to explain and could be interpreted as an extraordinarily large RV-activity correlation. We remain puzzled by this system.

HD 85440 — We obtain a reasonable planet fit for HD 85440. However, the RV measurements are highly correlated with the activity measurements (Pearson coefficient 0.929), and so we disfavor the planet, simply counting all variation as activityinduced jitter.

HD 100337-HD 100337 also shows a strong correlation between the activity and RV measurements (Pearson coefficient 0.789 ).

HD 193391-HD 193391 shows a strong correlation between the activity and RV measurements (Pearson coefficient 0.860).

$$
\text { B.10. } 1.6 \leqslant \mathrm{M}_{\star}<1.7 \mathrm{M}_{\odot}
$$

HD 12137-HD 12137 appears to be a binary system. After subtracting a linear trend, we find evidence of a 5000 day period planet. However, we are reluctant to subtract it due to the low number of observations. We simply subtract a line for now.

HD 45210-HD 45210 also shows a long-term linear trend, which we have subtracted out.

$$
\text { B.11. } \mathrm{M}_{\star}>1.7 \mathrm{M}_{\odot}
$$

HD 225021-HD 225021 shows evidence of a linear trend, which we have removed, resulting in an RV jitter of $8 \mathrm{~m} \mathrm{~s}^{-1}$ instead of $27 \mathrm{~m} \mathrm{~s}^{-1}$.

HD 31543-HD 31543 shows clear evidence of a binary companion. However, the final two observations have very large errors (10-20 $\mathrm{m} \mathrm{s}^{-1}$ ) and are the only two observations after the velocities have turned over and begin to increase again. Because of the large uncertainties for those measurements, the orbit is not well constrained and other solutions exist.

HD 44506-HD 45506 shows a coherent downward trend in the velocities, spanning a range of $100 \mathrm{~m} \mathrm{~s}^{-1}$. We have therefore subtracted a linear trend, which results in a jitter of $8 \mathrm{~m} \mathrm{~s}^{-1}$ (26 m s $\mathrm{m}^{-1}$ otherwise).

HD 64730-HD 64730 shows evidence of a stellar companion; however, it has only completed one velocity minimum. In addition, there are strong velocity variations that result in higheccentricity fits for this system. Due to the few observations, we cannot fully constrain the orbit and can merely report an RV jitter about a poor fit. The resulting RV jitter is $49 \mathrm{~m} \mathrm{~s}^{-1}$, beyond the range of the plots in Figures 4 and 5.

HD 96683-HD 96683 shows evidence of a long-term trend but with some slight curvature. We have opted to include the full Keplerian fit, which results in an RV rms of $7.4 \mathrm{~m} \mathrm{~s}^{-1}$, but note that this is likely an overfit. The RV rms about a linear trend is $40 \mathrm{~m} \mathrm{~s}^{-1}$, and about a parabola is $7.7 \mathrm{~m} \mathrm{~s}^{-1}$, indicating that the curvature is indeed significant.

\section{B.12. Stars Removed from the Sample}

Here we list the handful of stars that were removed from the sample along with the reasons for their removal.

HD 92855-The cadence of observations for HD 92855 is not high enough to provide a Keplerian fit to the $30 \mathrm{~km} \mathrm{~s}^{-1}$ variations in the RVs. We expect that such a high amplitude variation likely arises from a stellar companion of nearly equal mass. We are therefore suspicious of contamination in the RVs from the companion that also contributes to the poor fit.

HD 4741-HD 4741 shows evidence of a potentially longperiod stellar-mass companion (about $8 \mathrm{~km} \mathrm{~s}^{-1}$ variation). However, we have exactly 10 observations of this star over a span of $10 \mathrm{yr}$, but only 5 have any significant leverage, as the first 4 and final 3 observations are clustered in a period of about a week. This star was observed too infrequently in between those sets of observations that a Keplerian fit would be overfitting. Indeed, while we are able to fit a Keplerian that results in $7 \mathrm{~m} \mathrm{~s}^{-1}$ of jitter, the resulting eccentricity is likely unphysical $(e=0.99)$ and indicative of the fitter "chasing" a single point. We expect this best fit to be an overfit.

HD 202568 - HD 202568 has very large internal error bars for every RV measurement $\left(8 \mathrm{~m} \mathrm{~s}^{-1}\right)$. In addition, the observations span only one and a half years, giving only a very small time baseline on which to anchor the jitter.

HD 34957-HD 34957 has evidence of spectral contamination in that all spectral lines have blueward asymmetries. We remove this star as a double-lined binary.

HD 75732 - HD 75732 (also known as $55 \mathrm{Cnc}$ ) has five planets in the system, which are interacting gravitationally at a scale that is discernible in the RVs (Nelson et al. 2014). For that reason, a multiplanet Keplerian fit is not sufficient for this system, and it requires an $\mathrm{N}$-body dynamical model, as in Nelson et al. (2014), which is beyond this analysis. We therefore remove it from our sample, as we know we cannot recreate the best fit for this system. For reference, Nelson et al. (2014) measure a jitter from Keck to be 3 to $3.5 \mathrm{~m} \mathrm{~s}^{-1}$ depending on whether the high-cadence observations were treated as independent or perfectly correlated.

HD 16297-Despite meeting our threshold of having 10 or more observations, HD 16297 has large uncertainties on the velocity measurements prior to the Keck-HIRES upgrade (median $14.5 \mathrm{~m} \mathrm{~s}^{-1}$ ). When these points are removed, the remaining number of good observations is only six, and so we remove it from our sample.

HD 69076-HD 69076 has large amplitude variations $\left(20 \mathrm{~m} \mathrm{~s}^{-1}\right)$ that indicate the presence of a stellar companion on what appears to be a short orbit. However, we are unable to obtain a believable best-fit Keplerian for this system and so we are suspicious of spectral contamination that may be affecting the measured RVs.

HD 88656-Like HD 16297 above, HD 88656 does not meet our threshold for needing 10 or more observations once we remove the highly uncertain and clearly inconsistent velocities prior to the Keck upgrade.

\section{ORCID iDs}

Jacob K. Luhn (i) https://orcid.org/0000-0002-4927-9925

Jason T. Wright (1) https://orcid.org/0000-0001-6160-5888

Andrew W. Howard (i) https://orcid.org/0000-0001-

8638-0320

Howard Isaacson (iD https://orcid.org/0000-0002-0531-1073

\section{References}

Aigrain, S., Pont, F., \& Zucker, S. 2012, MNRAS, 419, 3147

Baines, E. K., Armstrong, J. T., \& van Belle, G. T. 2013, ApJL, 771, L17 Baluev, R. V., \& Beaugé, C. 2014, MNRAS, 439, 673

Barbieri, M., Alonso, R., Desidera, S., et al. 2009, A\&A, 503, 601 
Bastien, F. A., Stassun, K. G., \& Pepper, J. 2014a, ApJL, 788, L9 Bastien, F. A., Stassun, K. G., Pepper, J., et al. 2014b, AJ, 147, 29 Baudin, F., Barban, C., Belkacem, K., et al. 2011, A\&A, 529, A84 Boisse, I., Pepe, F., Perrier, C., et al. 2012, A\&A, 545, A55 Borucki, W. J., Koch, D., Basri, G., et al. 2010, Sci, 327, 977 Bouchy, F., Mayor, M., Lovis, C., et al. 2009, A\&A, 496, 527 Bouchy, F., Udry, S., Mayor, M., et al. 2005, A\&A, 444, L15 Bowler, B. P., Johnson, J. A., Marcy, G. W., et al. 2010, ApJ, 709, 396 Brewer, J. M., Fischer, D. A., Basu, S., Valenti, J. A., \& Piskunov, N. 2015, ApJ, 805, 126

Brewer, J. M., Fischer, D. A., Valenti, J. A., \& Piskunov, N. 2016, ApJS, 225,32

Brewer, J. M., Fischer, D. A., Valenti, J. A., \& Piskunov, N. 2017, ApJS, 230, 12

Bryan, M. L., Knutson, H. A., Howard, A. W., et al. 2016, ApJ, 821, 89

Butler, R. P., Marcy, G. W., Williams, E., et al. 1996, PASP, 108, 500

Butler, R. P., Vogt, S. S., Laughlin, G., et al. 2017, AJ, 153, 208

Butler, R. P., Wright, J. T., Marcy, G. W., et al. 2006, ApJ, 646, 505

Campbell, B., Walker, G. A. H., \& Yang, S. 1988, ApJ, 331, 902

Carter, J. A., Winn, J. N., Gilliland, R., \& Holman, M. J. 2009, ApJ, 696, 241

Chaplin, W. J., Houdek, G., Karoff, C., Elsworth, Y., \& New, R. 2009, A\&A, 500, L21

Corsaro, E., Fröhlich, H.-E., Bonanno, A., et al. 2013, MNRAS, 430, 2313

Corsaro, E., Stello, D., Huber, D., et al. 2012, ApJ, 757, 190

Courcol, B., Bouchy, F., Pepe, F., et al. 2015, A\&A, 581, A38

Crepp, J. R., Gonzales, E. J., Bechter, E. B., et al. 2016, ApJ, 831, 136

da Silva, R., Udry, S., Bouchy, F., et al. 2007, A\&A, 473, 323

Díaz, M. R., Jenkins, J. S., Tuomi, M., et al. 2018, AJ, 155, 126

Díaz, R. F., Santerne, A., Sahlmann, J., et al. 2012, A\&A, 538, A113

Díaz, R. F., Ségransan, D., Udry, S., et al. 2016, A\&A, 585, A134

Dressing, C. D., Charbonneau, D., Dumusque, X., et al. 2015, ApJ, 800, 135

Dumusque, X., Glenday, A., Phillips, D. F., et al. 2015, ApJL, 814, L21

Dumusque, X., Udry, S., Lovis, C., Santos, N. C., \& Monteiro, M. J. P. F. G. 2011, A\&A, 525, A140

Duncan, D. K., Vaughan, A. H., Wilson, O. C., et al. 1991, ApJS, 76, 383

Egeland, R., Soon, W., Baliunas, S., et al. 2017, ApJ, 835, 25

Endl, M., Cochran, W. D., Wittenmyer, R. A., \& Hatzes, A. P. 2006, AJ, 131, 3131

Feng, F., Tuomi, M., Jones, H. R. A., et al. 2017, AJ, 154, 135

Fischer, D., Driscoll, P., Isaacson, H., et al. 2009, ApJ, 703, 1545

Fischer, D. A., Laughlin, G., Marcy, G. W., et al. 2006, ApJ, 637, 1094

Fischer, D. A., Vogt, S. S., Marcy, G. W., et al. 2007, ApJ, 669, 1336

Fulton, B. J., Howard, A. W., Weiss, L. M., et al. 2016, ApJ, 830, 46

Galland, F., Lagrange, A.-M., Udry, S., et al. 2005, A\&A, 443, 337

Gaudi, B. S., \& Winn, J. N. 2007, ApJ, 655, 550

Ghezzi, L., Montet, B. T., \& Johnson, J. A. 2018, ApJ, 860, 109

Giguere, M. J., Fischer, D. A., Howard, A. W., et al. 2012, ApJ, 744, 4

Giguere, M. J., Fischer, D. A., Payne, M. J., et al. 2015, ApJ, 799, 89

Gregory, P. C., \& Fischer, D. A. 2010, MNRAS, 403, 731

Haghighipour, N., Butler, R. P., Rivera, E. J., Henry, G. W., \& Vogt, S. S. 2012, ApJ, 756, 91

Harakawa, H., Sato, B., Fischer, D. A., et al. 2010, ApJ, 715, 550

Harakawa, H., Sato, B., Omiya, M., et al. 2015, ApJ, 806, 5

Hatzes, A. P., \& Cochran, W. D. 1998, in ASP Conf. Ser. 154, Cool Stars,

Stellar Systems, and the Sun, ed. R. A. Donahue \& J. A. Bookbinder (San Francisco, CA: ASP), 311

Haywood, R. D., Collier Cameron, A., Queloz, D., et al. 2014, MNRAS, 443,2517

Haywood, R. D., Collier Cameron, A., Unruh, Y. C., et al. 2016, MNRAS, 457,3637

Hébrard, G., Arnold, L., Forveille, T., et al. 2016, A\&A, 588, A145

Hébrard, G., Bonfils, X., Ségransan, D., et al. 2010, A\&A, 513, A69

Hekker, S., Snellen, I. A. G., Aerts, C., et al. 2008, A\&A, 480, 215

Howard, A. W., Johnson, J. A., Marcy, G. W., et al. 2009, ApJ, 696, 75

Howard, A. W., Johnson, J. A., Marcy, G. W., et al. 2010, ApJ, 721, 1467

Howard, A. W., Johnson, J. A., Marcy, G. W., et al. 2011, ApJ, 726, 73

Howell, S. B., Sobeck, C., Haas, M., et al. 2014, PASP, 126, 398

Huber, D., Chaplin, W. J., Christensen-Dalsgaard, J., et al. 2013, ApJ, 767, 127

Isaacson, H., \& Fischer, D. 2010, ApJ, 725, 875

Jensen-Clem, R., Millar-Blanchaer, M., Mawet, D., et al. 2016, ApJ, 820, 111

Johnson, J. A., Aller, K. M., Howard, A. W., \& Crepp, J. R. 2010, PASP, 122, 905

Johnson, J. A., Butler, R. P., Marcy, G. W., et al. 2007a, ApJ, 670, 833

Johnson, J. A., Clanton, C., Howard, A. W., et al. 2011, ApJS, 197, 26

Johnson, J. A., Fischer, D. A., Marcy, G. W., et al. 2007b, ApJ, 665, 785

Johnson, J. A., Marcy, G. W., Fischer, D. A., et al. 2006, ApJ, 652, 1724

Johnson, J. A., Marcy, G. W., Fischer, D. A., et al. 2008, ApJ, 675, 784
Jones, H. R. A., Butler, R. P., Tinney, C. G., et al. 2010, MNRAS, 403, 1703 Jones, M. I., Jenkins, J. S., Brahm, R., et al. 2016, A\&A, 590, A38 Kallinger, T., De Ridder, J., Hekker, S., et al. 2014, A\&A, 570, A41 Kane, S. R., Howell, S. B., Horch, E. P., et al. 2014, ApJ, 785, 93

Kippenhahn, R., Weigert, A., \& Weiss, A. 2012, Stellar Structure and Evolution (Berlin: Springer)

Kjeldsen, H., \& Bedding, T. R. 1995, A\&A, 293, 87

Kjeldsen, H., \& Bedding, T. R. 2011, A\&A, 529, L8

Kraft, R. P. 1967, ApJ, 150, 551

Latham, D. W., Mazeh, T., Stefanik, R. P., Mayor, M., \& Burki, G. 1989, Natur, 339, 38

Lloyd, J. P. 2011, ApJL, 739, L49

Lloyd, J. P. 2013, ApJL, 774, L2

Lo Curto, G., Mayor, M., Benz, W., et al. 2010, A\&A, 512, A48

Lovis, C., Dumusque, X., Santos, N. C., et al. 2011, arXiv:1107.5325

Lovis, C., Mayor, M., Pepe, F., et al. 2006, Natur, 441, 305

Luhn, J. K., Bastien, F. A., Wright, J. T., et al. 2019, AJ, 157, 149

Luhn, J. K., Wright, J. T., \& Isaacson, H. 2020, AJ, 159, 236

Mamajek, E. E., \& Hillenbrand, L. A. 2008, ApJ, 687, 1264

Mayor, M., Marmier, M., Lovis, C., et al. 2011, arXiv:1109.2497

Mayor, M., \& Queloz, D. 1995, Natur, 378, 355

Mayor, M., Udry, S., Naef, D., et al. 2004, A\&A, 415, 391

Medina, A. A., Johnson, J. A., Eastman, J. D., \& Cargile, P. A. 2018, ApJ, 867,32

Melo, C., Santos, N. C., Gieren, W., et al. 2007, A\&A, 467, 721

Meschiari, S., Laughlin, G., Vogt, S. S., et al. 2011, ApJ, 727, 117

Meunier, N., \& Lagrange, A.-M. 2019, A\&A, 625, L6

Meunier, N., Lagrange, A.-M., Borgniet, S., \& Rieutord, M. 2015, A\&A, 583, A118

Meunier, N., Lagrange, A.-M., \& Desort, M. 2010, A\&A, 519, A66

Meyer, M. R., Hillenbrand, L. A., Backman, D., et al. 2006, PASP, 118, 1690

Milbourne, T. W., Haywood, R. D., Phillips, D. F., et al. 2019, ApJ, 874, 107

Minniti, D., Butler, R. P., López-Morales, M., et al. 2009, ApJ, 693, 1424

Mood, A. M., Graybill, F. A., \& Boes, D. C. 1974, Introduction to the Theory of Statistics (3rd ed.; New York: McGraw-Hill)

Mordasini, C., Mayor, M., Udry, S., et al. 2011, A\&A, 526, A111

Moutou, C., Hébrard, G., Bouchy, F., et al. 2009, A\&A, 498, L5

Nelson, B. E., Ford, E. B., Wright, J. T., et al. 2014, MNRAS, 441, 442

Noyes, R. W., Hartmann, L. W., Baliunas, S. L., Duncan, D. K., \& Vaughan, A. H. 1984, ApJ, 279, 763

Parker, E. N. 1955, ApJ, 122, 293

Paxton, B., Cantiello, M., Arras, P., et al. 2013, ApJS, 208, 4

Peek, K. M. G., Johnson, J. A., Fischer, D. A., et al. 2009, PASP, 121, 613

Pepe, F., Lovis, C., Ségransan, D., et al. 2011, A\&A, 534, A58

Pepper, J., Rodriguez, J. E., Collins, K. A., et al. 2017, AJ, 153, 215

Pilyavsky, G., Mahadevan, S., Kane, S. R., et al. 2011, ApJ, 743, 162

Rast, M. P. 2003, ApJ, 597, 1200

Ricker, G. R., Winn, J. N., Vanderspek, R., et al. 2014, Proc. SPIE, 9143, 914320

Rivera, E. J., Butler, R. P., Vogt, S. S., et al. 2010, ApJ, 708, 1492

Robinson, S. E., Laughlin, G., Vogt, S. S., et al. 2007, ApJ, 670, 1391

Rowan, D., Meschiari, S., Laughlin, G., et al. 2016, ApJ, 817, 104

Rutten, R. G. M. 1984, A\&A, 130, 353

Saar, S. H., Butler, R. P., \& Marcy, G. W. 1998, ApJL, 498, L153

Santos, N. C., Mayor, M., Naef, D., et al. 2000, A\&A, 361, 265

Santos, N. C., Mayor, M., Naef, D., et al. 2001, A\&A, 379, 999

Santos, N. C., Udry, S., Bouchy, F., et al. 2008, A\&A, 487, 369

Sato, B., Fischer, D. A., Ida, S., et al. 2009, ApJ, 703, 671

Sato, B., Omiya, M., Harakawa, H., et al. 2012, PASJ, 64, 135

Schnupp, C., Bergfors, C., Brandner, W., et al. 2010, A\&A, 516, A21

Schwarzschild, M. 1975, ApJ, 195, 137

Shah, S. P., Wright, J. T., Isaacson, H., Howard, A., \& Curtis, J. L. 2018 ApJL, 863, L26

Skumanich, A. 1972, ApJ, 171, 565

Stello, D., Huber, D., Grundahl, F., et al. 2017, MNRAS, 472, 4110

Tamuz, O., Ségransan, D., Udry, S., et al. 2008, A\&A, 480, L33

Tan, X., Payne, M. J., Lee, M. H., et al. 2013, ApJ, 777, 101

Tinney, C. G., Butler, R. P., Jones, H. R. A., et al. 2011, ApJ, 727, 103

Torres, G., Winn, J. N., \& Holman, M. J. 2008, ApJ, 677, 1324

Trampedach, R., Asplund, M., Collet, R., Nordlund, A., \& Stein, R. F. 2013, ApJ, 769, 18

Udry, S., Dumusque, X., Lovis, C., et al. 2019, A\&A, 622, A37

Udry, S., Mayor, M., Clausen, J. V., et al. 2003, A\&A, 407, 679

Udry, S., Mayor, M., Naef, D., et al. 2002, A\&A, 390, 267

Valenti, J. A., Fischer, D., Marcy, G. W., et al. 2009, ApJ, 702, 989

Valenti, J. A., \& Fischer, D. A. 2005, ApJS, 159, 141 
Van Grootel, V., Gillon, M., Valencia, D., et al. 2014, ApJ, 786, 2 van Saders, J. L., \& Pinsonneault, M. H. 2013, ApJ, 776, 67

Vogt, S. S., Butler, R. P., Marcy, G. W., et al. 2005, ApJ, 632, 638

Vogt, S. S., Wittenmyer, R. A., Butler, R. P., et al. 2010, ApJ, 708, 1366

Wang, S. X., Wright, J. T., Cochran, W., et al. 2012, ApJ, 761, 46

Weiss, L. M., \& Marcy, G. W. 2014, ApJL, 783, L6

Williams, S. J., Gies, D. R., Hillwig, T. C., McSwain, M. V., \& Huang, W. 2013, AJ, 145,29

Wilson, O. C. 1963 , ApJ, 138, 832

Wittenmyer, R. A., Endl, M., \& Cochran, W. D. 2007, ApJ, 654, 625

Wittenmyer, R. A., Endl, M., Cochran, W. D., Levison, H. F., \& Henry, G. W. 2009, ApJS, 182, 97

Wittenmyer, R. A., Horner, J., Tinney, C. G., et al. 2014, ApJ, 783, 103

Wittenmyer, R. A., Horner, J., Tuomi, M., et al. 2012, ApJ, 753, 169
Wittenmyer, R. A., Johnson, J., Wang, L., \& Endl, M. 2011, in AIP Conf. Ser. 1331, Planetary Systems beyond the Main Sequence, ed. S. Schuh, H. Drechsel, \& U. Heber (Melville, NY: AIP), 117

Wittenmyer, R. A., Wang, S., Horner, J., et al. 2013, ApJS, 208, 2

Wolszczan, A., \& Frail, D. A. 1992, Natur, 355, 145

Wright, J. T. 2004, AJ, 128, 1273

Wright, J. T. 2005, PASP, 117, 657

Wright, J. T. 2015, in Proc. Coll. Twenty Years of Giant Exoplanets Held at Observatoire de Haute Provence, ed. I. Boisse et al. (Saint-Michell'Observatoire: Observatoire de Haute-Provence), 8

Wright, J. T., \& Howard, A. W. 2009, ApJS, 182, 205

Wright, J. T., Marcy, G. W., Butler, R. P., et al. 2008, ApJL, 683, L63

Wright, J. T., Upadhyay, S., Marcy, G. W., et al. 2009, ApJ, 693, 1084

Wright, J. T., Veras, D., Ford, E. B., et al. 2011, ApJ, 730, 93 